\title{
RENDICONTI DEL CIRCOLO MATEMATICO DI PALERMO.
}

\section{L'OPERA GEOMETRICA DI A. MANNHEIM.}

\author{
Studio di Gino Loria (Genova).
}

\begin{abstract}
"Si confessi dunque volentieri, che la provincia, sulla quale domina siccome Reina l'Analisi, è molto più estesa e più vasta di quella che appartiene alla Sintesi; ma non s'inferisca da tal preminenza, che l'altra debbasi abbandonare, l'altra tanto benemerita e per tanti secoli della Geometria antica che serve anche oggidi all'Algebra medesima di util compagna ....
\end{abstract}

G. F. MALIATTI.

Quantunque la mirabile produzione scientifica di M. Chasles non abbia ancora trovato il suo storico, pure, un semplice esane superficiale palesa in essa tre facce. $\mathrm{E}$, infatti, noto come una sua parte preponderante sia rappresentata dalle memorabili ricerche storiche di quel grande; una seconda, non meno cospicua, dagli studî di Geometria projettiva, inteso questo vocabolo nella sua accettazione più ampia; un'ultima, infine, forse meno estesa, ma per fermo non meno importante, abbraccia le ricerche di Cinematica pura (o Geometria cinematica, adottando la proposta fatta nel 1859 dal Terquem), teoria del movimento indipendentemente dalle cause che lo producono (forze) e dal tempo durante il quale esso avviene. Mentre Chasles come storico trovo, non nella propria patria, ma nella terra di AмLeтo, un discepolo degno di calcarne le orme gloriose ${ }^{\mathrm{I}}$ ), mentre in E. DE Jonquítres trovó chi fu capace di proseguire e completare le sue indagini sulle figure algebriche ${ }^{2}$ ), gli è nell'eminente geometra, di cui intendiamo di occuparci nel presente lavoro e per la cui recente dipartita è così vivo il dolore, che egli ravvisó la persona destinata a svolgere in modo originale e portare alle loro più lontane conseguenze le feconde sue idee sulla dottrina geometrica del movimento. Egli ebbe cosi netta la visione della missione che questi era destinato a compiere da considerarlo e designarlo come proprio "figlio spirituale "; ma per quanto

I) “Come discepolo di Chasles », scrive lo Zeuthen nella prefazione alla sua grande opera sopra le ricerche dei Greci intorno alle coniche, "da tempo io m'interesso ai risultati ottenuti nell'antichità ».

2) Cfr. il mio studio: L'Guvre mathématique d'ERNEST DE JonguiEres [Bibliotheca Mathematica, serie III, tomo III (1902), pp. 276-322]. 
acuto e lungimirante fosse il suo sguardo, per fermo non avrà saputo prevedere la mole elegante del grande edificio che questi avrebbe architettato ed eretto.

\section{I. - Una Primavera rigogliosa.}

\section{Vittorio Amedeo Mannheim nacque a Parigi il 17 Luglio $183 \mathrm{I}$.}

Fino dalla più tenera età manifestó attitudini spiccatissime per le scienze esatte, giacchè a dieci anni, pur essendo il più giovane della propria classe, fu il solo che riuscisse a risolvere un problema di geometria proposto dal professore.

Compiuto il corso degli studi costituenti il programma dell'( Institution Martelet », a sedici anni venne ammesso all' 'École centrale »; ma tale istituto egli abbandonò ben presto pel Liceo Carlomagno, ove, sotto la guida amorosa e sapiente di E. Catalan, si preparó all'ammissione nella Scuola politecnica: quel celebre geometra ebbe ragione di andare orgoglioso di un discepolo che fu accolto ( 1848 ) nella più famosa scuola del mondo, essendo sempre il più giovane del proprio corso.

Uscitone due anni dopo sottotenente d'artiglieria, venne destinato alla scuola d'applicazione di Metz; ed appunto in tale epoca ided una modificazione al regolo calcolatore (cfr. gli articoli [4], [6] ${ }^{3}$ ), che fu adottata dal corpo a cui apparteneva il Mannheim e poi generalmente, ottenne una distinzione all'Esposizione Universale del 1855 , riscosse le lodi di un giudice competente ${ }^{4}$ ) e servi di modello nella costruzione di altri apparati congeneri ${ }^{5}$ ).

Appunto ai primi tempi (Gennajo I85I) del soggiorno a Metz del giovane sottotenente d'artiglieria appartiene una delle prime sue pubblicazioni geometriche [3]. Essa contiene svariate applicazioni della trasformazione per polarità rispetto ad un cerchio alla ricerca di nuove proprietà descrittive delle figure piane; da ultimo l'Autore cerca la relazione che passa fra la distanza di due punti e qualche elemento metrico della figura corrispondente e la trova almeno in alcuni casi speciali: ciò va notato perchè mostra come egli sin da allora abbia rivolta la propria attenzione alla polarizzazione delle

3) I numeri posti in parentesi quadra [ ] servono di richiamo ai lavori elencati alla fine dell'attuale scritto.

4) «Il sig. Mannheim ha fatto eseguire un nuovo ed ingegnoso regolo, per mezzo del quale si ha un'esattezza doppia di quella data da eguali regoli ordinarii, non solo per la moltiplicazione e divisione, ma benanco per ogni proporzione, per $i$ quadrati cubi, radici quadrate, cubiche $e$ simili». [Q. Sella, Teorica e pratica del regolo calcolatore, Torino 1859 , p. I00; II ${ }^{\mathrm{a}}$ ed. italiana, T'orino 1886 , p. 102].

5) Cfr. P. Rozé, Théorie et usage de la règle à calculs (règle des écoles, rìgle Mannherm), Paris 1907, p. 2. Va aggiunto che nel tomo XIII (1854), p. 36, delle Nouvelles Annales de Mathématiques è detto che il nostro autore fece costruire " une Règle à calcul cylindrique, plus commode, plus exacte, que sa règle plate ». Notiamo qui anche avere egli più tardi (1857) ideato un altro utile strumento ([67], [74]), che riscosse l'approvazione di Poncelet e il cui modello originale può tuttora vedersi nel "Conservatoire des Arts et Métiers » di Parigi. 
proprietà metriche delle figure, argomento che (come vedremo) egli non perdette mai di vista durante tutta la sua vita.

Promosso nel 1852 tenente d'artiglieria, il Mannheim ando peregrinando per varie guarnigioni; delle sue ottime qualita militari rende fede il fatto che a Parigi, durante un'epidemia colèrica, col proprio contegno fermo egli seppe tenere alto il morale delle truppe affidategli, mentre alcune sue osservazioni sparse, alcune questioni proposte ed alcune eleganti soluzioni ${ }^{6}$ ) possono citarsi come prova del non avere egli mai abbandonati del tutto gli studì prediletti. D'altronde, appunto a tale periodo appartengono altri buoni suoi lavori, i quali meritano qualche considerazione da parte di chi intende seguire passo passo l'evoluzione delle sue idee matematiche.

Nell'uno [ 5 ] trovasi applicata un'elegante considerazione stereometrica (senza dubbio ispirata da MONGE) alla dimostrazione dell'essere formato da quattro rette concorrenti in un punto il luogo dei centri delle circonferenze seganti sotto angoli eguali tre circonferenze date.

In altro [7] Mannherm enuncia una proprietà dell'inviluppo di una sfera costantemente tangente a due piani ed una sfera, preludendo cosi ad altre ricerche che poco dopo attrassero la sua attenzione.

Finalmente in un terzo [8] egli dimostra che l'isottica di due circonferenze è costituita da un certo numero di lumache di PASCAL; a tale scopo egli adopera considerazioni di pertinenza della Geometria cinematica, le quali provano come sin dal I856 egli fosse famigliare con tale disciplina e spiegano come poco dopo egli potesse venire considerato come il rappresentante di essa ${ }^{7}$ ).

Nel i855 troviamo il Mannheim a Marsiglia, nel punto in cui, essendo stato destinato in Crimea, aveva già imbarcato la propria compagnia e stava per salpare. $\mathrm{Ma}$ la presa di Sebastopoli e la pace che ne fu il corollario sospese la partenza di nuovi rinforzi e risparmiò forse alla patria ed alla scienza il sagrificio d'una esistenza preziosa!

Durante il soggiorno a Marsiglia, prolungatosi parecchi mesi, il protagonista della storia che stiamo narrando pote riprendere con agio e tranquillitd relativi gli studi interrotti; le risultanze dei quali leggonsi in alcuni lavori, su cui è nostro dovere arrestarci qualche istante.

In quello di più antica data [9] ${ }^{8}$ ) egli ritorna, per svolgerlo con l'ampiezza necessaria, su un tema già toccato in una sua precedente pubblicazione [3], cioè la ricerca di un metodo per trasformare le proprietà metriche delle figure piane col mezzo d'una polarità rispetto ad un cerchio, ricerca di cui la soluzione doveva sembrare del massimo valore nel tempo in cui scriveva il Mannheim, cioè in un'epoca in cui il regno della Geometria era governato da due dittatori: Poncelet, di cui sono giustamente rinomati i contributi alla teoria della polarità, e CHAsLes, di cui è notorio l'affetto per le rela-

6) Nouvelles Annales de Mathématiques, Ière série: t. XII (1853), pp. I07-108; t. XIII (1854), pp. 35-36, 210-211, 266-267; t. XVI (1857), pp. 187-189.

7) Nouvelles Annales de Mathématiques, Ière série, t. XVIII (I859), p. 445.

8) Cfr. [184], pp. 477-485. 
zioni metriche. Per risolvere quella questione il nostro autore generalizza la formola che già rilevammo nella chiusa di quella pubblicazione [3] e cosi ottiene la relazione generale che intercede tra la distanza di due punti di una figura piana e gli elementi metrici della figura polare ${ }^{9}$ ). Molteplici applicazioni fattene dall'autore ${ }^{10}$ ) chiariscono il modo di servirsi di tale notevole relazione; ci dispensiamo dal riferire le proposizioni cosi ottenute dal Mannheim essendo in parte note ed in parte di enunciazione complicata: cio che invece va rilevato si è che con questo suo lavoro giovanile egli non ha esaurito il relativo soggetto, come vedremo meglio in seguito. - Gli altri lavori composti dal nostro geometra mentre era in servizio militare attivo trattano temi che rientrano nel vasto campo di studi a cui egli doveva poi dedicare la miglior parte della propria attività, cioè la costruzione delle normali e dei centri di curvatura di determinati luoghi geometrici, ovvero dei punti di contatto di speciali inviluppi. Fra i risultati più generali ottenuti nel più antico di essi [Io] citiamo ad esempio il seguente: Una retta $R$ tocchi in $r$ una curva fissa $U$ ed incontri (in a e b) due altre curve $A, B$; sia $m$ un punto del segmento $a b$ tale che sia $\frac{a m}{m b}=\lambda$ (cost.); la normale alla curva $(m)$, luogo dei punti $m$, divide nello stesso rapporto $\lambda$ il segmento determinato sulla normale in $r$ a $U$ dalle normali in $a, b$ alle curve $A, B^{{ }^{\mathrm{I}}}$ ). Nè vanno dimenticate varie costruzioni del centro di curvatura di una conica ${ }^{\mathbf{1 2}}$ ), da inscriversi nel ricco elenco dei contributi dati dal Mannheim alla teoria delle curve di second'ordine.-Tra le questioni risolte in altro [12] ${ }^{\text {I3 }}$ ) dei riferiti lavori, notiamo la costruzione della tangente al luogo geometrico del punto in cui si tagliano le tangenti in due punti $a, b$ di due date curve $A, B$ scelti in modo che $b$ sia il piede di una normale condotta da $a$ a $B$; fra le conseguenze speciali segnaliamo una costruzione semplice del raggio di curvatura dell'ovale di CarTEsio ${ }^{\mathrm{I}}$ ) (in particolare di una conica) ed altre dei punti di una caustica: soggetto quest'ultimo sul quale il Mannherm doveva ritornare [ $\mathrm{I} 60$ ] tanti anni appresso. Nello stesso lavoro egli fa cenno della seguente questione, che il marchese DE L'HôpITAL aveva incompletamente risolta nella sua "Analyse des infinitement petits": date due curve $A, A^{\prime}$ ed un punto qualunque $f$ del loro piano, si conduca per $f$ una trasversale arbitraria a segare quelle curve in $a, a^{\prime}$; determinare la tangente del luogo del punto in cui tagliansi le tangenti in $a, a^{\prime}$ alle curve date; ed in uno posteriore [14] ${ }^{\mathrm{I}}$ ) la risolve in due modi entrambi notevoli e che conservano la propria validità nell'ipotesi che $f$ vada all'infinito.

9) Non potendo qui riferirla, noteremo che una delle forme sotto cui puó scriversi ebbe l'onore di essere riferita da Chasles nel suo Rapport sur les progrès de la Géométrie (Paris, 1870), p. 294.

10) Cfr. [152], p. I9I e [184], p. 26.

II) Cfr. [152], p. I72.

$\left.{ }^{2}\right)$ Segnaliamo, a mo' d'esempio, quella emergente dalla proposizione seguente: la parabola tangente agli assi di un'ellisse nonchè alla normale ed alla tangente in un punto della curva, tocca tale normale nel relativo centro di curvatura.

13) Il tema di esso è chiaramente indicato dal titolo. Cfr. anche [184], pp. 485-492.

14) Cfr. [152], p. 209.

15) Cfr. [184], pp. 492-500. 
Analoghe considerazioni infinitesimali guidarono il nostro autore a costruire il centro di curvatura in un punto qualunque della curva che nasce da una curva piana dividendone le ordinate in un rapporto costante; ne deduce che: se una curva ammette un diametro rettilineo, $i$ raggi di curvatura negli estremi di una corda ad esso conjugata stanno fra di loro come $i$ cubi delle relative tangenti limitate al loro punto d'intersezione ${ }^{\mathbf{1 6}}$ ), e in particolare che: $i$ raggi di curvatura in due punti qualunque di una conica stanno fra di loro come $i$ cubi delle corrispondenti tangenti limitate al loro punto comune ${ }^{17}$ ). Ora è degno di nota che tale elegante proprietà delle coniche era stata avvertita, prima che il Mannheim la scoprisse, dall'UmpFenbach ${ }^{18}$ ) e dal Liouville ${ }^{\text {19}}$ ), mentre lo fu poi dal Peaucellier ${ }^{20}$ ). Il Mannheim la riottenne più tardi, assieme ad altre concernenti le cubiche piane, deducendola dà quest'altra, da lui semplicemente enunciata ${ }^{21}$ ): Una

r6) Tale proposizione si puo dimostrare agevolmente, ricorrendo alle seguenti espressioni per il raggio di curvatura $R$ e per la tangente $T$ in assi cartesiani inclinati fra loro dell'angolo $\omega:$

$$
R=\frac{\left(\mathrm{I}+y^{\prime} \cos \omega+y^{\prime 2}\right)^{\frac{3}{2}}}{y^{\prime \prime} \operatorname{sen} \omega}, \quad T=\frac{y\left(\mathrm{I}+2 y^{\prime} \cos \omega+y^{\prime 2}\right)^{\frac{1}{2}}}{y^{\prime}} .
$$

Infatti, da queste si trae, in valore assoluto,

$$
R=\frac{T^{3} y^{3}}{y^{\prime 3} y^{\prime \prime} \operatorname{sen} \omega} \text {. }
$$

Ora, se la curva che si considera ha per diametro $O x$ con le corde conjugate parallele a $O y$, cambiando il segno di $y$ (epperò di $y^{\prime}, y^{\prime \prime}$ ) e chiamando $R_{1}, T_{1}$ i corrispondenti valori di $R, T$, si ha, ancora in valore assoluto,

onde, con la stessa clausola,

$$
R_{1}=\frac{T_{1}^{3} y^{3}}{y^{\prime 3} y^{\prime \prime} \operatorname{sen} \omega}
$$

$$
\frac{R}{R_{\mathrm{I}}}=\frac{T^{3}}{T_{\mathrm{I}}^{3}} \text {. }
$$

${ }^{17}$ ) Questa proposizione si può dimostrare applicando le formole $(\alpha)$ della nota $\left.{ }^{\text {I6 }}\right)$ ai punti $(p, o)$, $(0, q)$ della conica

$$
\left(\frac{x}{p}+\frac{y}{q}-\mathrm{I}\right)+\lambda x y=0 .
$$

18) Ein Lebrsatz von Kegelschnitten [Journal für die reine und angewandte Mathematik, Bd. XXX (1846), pp. 95-96].

19) Sur une propriété des sections coniques [Journal de Mathématiques pures et appliquées, Ière série, t. IX (1844), pp. 350-352].

20) Relation entre les rayons de courbure d'une courbe et de sa perspective, et considérations sur les foyers des lunettes [Nouvelles Annales de Mathématiques, İ̉re série, t. XX (I86I), pp. 427-430].

${ }^{21}$ La via per la quale egli vi giunse risulta da [I52], p. 2I4 e [184], pp. 55-56. Con la scorta dell'analisi la si puó dimostrare semplicemente come segue: Riferendo la curva $(A)$ ad un sistema di coordinate polari $r, \omega$ col polo in $O$ e ponendo $r=\frac{\mathbf{I}}{u}$, si ha facilmente:

$$
\cos \alpha=\frac{u}{\left(u^{2}+u^{2}\right)^{\frac{1}{2}}}, \quad p=\frac{\left(u^{2}+u^{22}\right)^{\frac{3}{2}}}{u^{3}\left(u+u^{\prime \prime}\right)},
$$


trasversale condotta per un punto $O$ incontra in $A, A_{1}, A_{2}, \ldots$ le curve fisse $(A),\left(A_{1}\right)$, $\left(A_{2}\right), \ldots$; si prende sulla trasversale un punto $M$ tale che si abbia

$$
\frac{\lambda}{O A}+\frac{\lambda_{1}}{O A_{1}}+\frac{\lambda_{2}}{O A_{2}}+\cdots=\frac{\mu}{O M}
$$

ove $\lambda, \lambda_{1}, \lambda_{2}, \ldots, \mu$ sono costanti date. Quando la trasversale ruota attorno ad $O$, il punto $M$ descrive una curva $(M)$ tale che

$$
\frac{\lambda}{\rho \cos ^{3} \alpha}+\frac{\lambda_{1}}{\rho_{1} \cos ^{3} \alpha_{1}}+\frac{\lambda_{2}}{\rho_{2} \cos ^{3} \alpha_{2}}+\cdots=\frac{\mu}{R \cos ^{3} \varphi^{\prime}},
$$

ove $p$ é il raggio di curvatura della curva $(A)$ in $A, e$ a l'angolo che la relativa normale forma con quella trastersale, mentre $c_{1}, \rho_{2}, \ldots, R e \alpha_{1}, \alpha_{2}, \ldots, \phi$ banno significati analoghi per le curve $\left(A_{\mathrm{s}}\right),\left(A_{2}\right), \ldots,(M)$. - Per determinare la genesi dell'ultimo [r3] dei lavori costituenti il gruppo succitato, fa mestieri ricordare come ABEL Transon abbia scoperta una procedura assai semplice per determinare il raggio di curvatura della trajettoria di un punto appartenente ad una figura piana mobile nel proprio piano con una legge determinata ${ }^{22}$ ) e come CHASLes ne abbia subito ${ }^{23}$ ) rivendicata a sè stesso la priorità e poco dopo fatta conoscere la sua "Construction des rayons de courbure des courbes décrites dans le mouvement d'une figure plane qui glisse sur son plan ${ }^{24}$ ). Ivi, fra l'altro, è notato che il moto di una figura piana nel proprio piano puó intendersi ottenuto facendo ruzzolare una curva su di un'altra e che, quando il moto è infinitesimo, a tali curve è lecito sostituire i corrispondenti cerchi di curvatura. Donde emerge la possibilita di applicare alla questione enunciata nel titolo di quella memoria di ChasLes una nota formola ${ }^{25}$ ) che va sotto il nome di Savary ${ }^{25}$ ). Ora è appunto di tale so-

epperò :

Similmente :

$$
\frac{\mathbf{I}}{\rho \cos ^{3} \alpha}=u+u^{\prime \prime}
$$

onde

$$
\frac{\mathrm{I}}{\rho_{1} \cos ^{3} \alpha_{1}}=u_{1}+u_{1}^{\prime \prime}, \quad \frac{\mathrm{I}}{\rho_{2} \cos ^{3} \alpha_{2}}=u_{2}+u_{2}^{\prime \prime}, \text { ecc. }
$$

$$
\frac{\lambda}{\rho_{1} \cos ^{3} \alpha}+\frac{\lambda_{1}}{\rho_{1} \cos ^{3} \alpha_{1}}+\frac{\lambda_{2}}{\rho_{2} \cos ^{3} \alpha_{2}}+\ldots=\left(\lambda u+\lambda_{1} u_{1}+\lambda_{2} u_{2}+\ldots\right)+\left(\lambda u+\lambda_{1} u_{1}+\lambda_{2} u_{2}+\ldots\right)^{\prime \prime}
$$

e, per l'ipotetica relazione (I), $=\frac{\mu}{R \cos ^{3} \varphi}$, secondo l'enunciato.

22) Abel Transon, Methode geométrique pour les rayons de courbure d'une certaine classe de courbes [Journal de Mathématiques pures et appliquées, Ière série, t. X (1845), pp. 148-I57].

23) Cfr. Arel Transon, Nota citata ${ }^{22}$ ), pp. i56-is7 (Note de M. Chasles).

24) Journal de Mathématiques pures et appliquées, Ière série, t. X (1845), pp. 204-208.

25) Cfr. Leroy, Cours de Géométrie descriptive, $2^{\mathrm{e}}$ édition (Paris, 1862), p. 386.

26) Mannheim ha osservato ([152], p. 176, e [184], p. 25) che in realtà essa appartiene ad EuIERO [v. la memoria Supplementium de figura dentium rotarum pubblicata in Novi Commentarii Academiae Petropolitanae, t. XI ( 1765$)$, p. 209]. Essa e le relative costruzioni si trovano esposte in un altro suo lavoro [17], il quale va anche segnalato per alcune eleganti proprietà della catenaria, parte nuove, parte dovute a LaMarle e Poinsot, dimostrate con procedimento uniforme. Cfr. anche la risposta data nel L'Intermédiaire des Mathématiciens [t. IX (Igo2), pp. I20-I 22] sotto il pseudonimo di "CANON 》 alla "Question 475 » proposta da “ROSACE» (=E. CesAro). 
stituzione che il MaNNHEIm profittó per trattare [13] questioni dello stesso tipo, ad esempio la seguente: supposto che un triangolo variabile di forma e grandezza si muova nel proprio piano con data legge, dai centri di curvatura degli elementi descritti da due vertici dedurre quello dell'elemento descritto dal terzo. Fra gli speciali risultati da lui ottenuti notiamo un bel teorema di ChasLes relativo al centro di curvatura dell'ellisse, l'estensione ad un asteroide obliquo di un'elegante proptietà scoperta dal LamarLe nell'asteroide regolare ed alcune proprietà della curva di Delaunay e delle linee reptorie del BERNOULLI.

\section{II. - Un'Estate feconda.}

Nel 1859 il Mannheim, già capitano, era applicato alla Fabbrica d'armi di Châtellerault. Un posto di ripetitore alla Scuola politecnica essendosi reso vacante, il Consiglio direttivo, accogliendo il suggerimento d'un illustre geometra, il LAMÉ, chiamó il Mannheim ad occuparlo: questi potè cosi tornare, in qualità d'insegnante, nel famoso Istituto che lo aveva annoverato fra i propri migliori discepoli e nel quale doveva rimanere per quasi tutta la sua vita, prima come esaminatore d'ammissione (I863), poi (I864I9OI) come successore di J. DE LA Gournerie nella cattedra fondata ed illustrata da Monge. Egli lo lasció temporaneamente soltanto durante un breve periodo, per seguire nel i 860 la spedizione scientifica di Costantina, nel corso della quale egli fece delle osservazioni di fisica che furono reputate degne di venire inserite in un'importante pubblicazione periodica ${ }^{27}$ ); va eziandio ricordato che, durante l'assedio sofferto da Parigi nel I87o, egli comandó la batteria della Scuola politecnica e che, nel sanguinoso periodo della Comune, egli abbandonó la capitale per riprendere il proprio insegnamento a Tours, provvisoria sede di quella Scuola.

Trasferito a Parigi, esonerato dalle quotidiane incombenze militari, il Mannheim si dedicó completamente alla scienza a lui caramente diletta ${ }^{28}$ ): le pagine seguenti diranno quale felice ispirazione abbia avuto il LAMÉ nello strappare dalla gamella chi poteva e doveva divenire uno degli uomini di cui oggi la Francia va giustamente orgogliosa!

Per dare un po' d'ordine alla nostra esposizione dell'opera geometrica del MannHeIm ne raggrupperemo $i$ lavori in un modo che ci sembra conforme alla loro natura; riconosciamo peró per primi che la nostra classificazione, suggerita esclusivamente da ragioni d'opportunità, apparirà per fermo artificiale a chi, studiando tutta la produzione del Nostro geometra, avvertirà il carattere che possiede di sostanziale unita, nella grande varietà de' suoi aspetti.

${ }^{27)}$ Franges mobiles incolores observées pendant l'éclipse de soleil du 18 juillet 1860 [Annales de Chimie, t. LX (1860), pp. 207-210].

28) L'antico ufficiale d'artiglieria si ritrova soltanto nell'articolo: Sur le tir lorsque le but est élevié au dessus de l'horizon, inserito nel $t$. VII (1876) della Revue d'Artillerie. 


\section{Polarità.}

La trasformazione per polari reciproche, che vedemmo interessare il MANNHEIM sin dagli albori della sua carriera scientifica, continuó ad occuparlo ancora per gran parte della sua vita. Lo prova anzitutto uno scritterello [I6] in cui egli insegna come scegliere il cerchio direttore d'una polarità affinchè questa muti una conica in altra dotata di proprieta prestabilite; e lo conferma una elegante proposizione metrica ${ }^{29}$ ) da lui proposta come esercizio, e che, per sua dichiarazione [I 82], egli ottenne, col mezzo di una duplice polarizzazione, da un teorema di Duhamel. Ma i risultati più cospicui delle sue indagini sopra tale argomento trovansi consegnati in un esteso lavoro [39] il cui fine è la ricerca di ció che divengono, per effetto d'una trasformazione polare, le proprietà di curvatura delle linee (piane e sghembe) e delle superficie. Le formole a cui giunse il Mannheim non sono abbastanza semplici per essere qui riferite; ma per dimostrarne l'utilità citeremo due delle conseguenze che egli ne trasse. Dalla proposizione, da lui dimostrata altrove [28]: "se una curva di terźordine è inscritta e circoscritta ad un triangolo, il prodotto dei raggi di curvatura nei vertici eguaglia il cubo del raggio del cerchio circoscritto al triangolo" ${ }^{30}$ ), egli dedusse che: "se una curva di terza classe è inscritta e circoscritta ad un triangolo, il prodotto dei raggi di curvatura nei vertici è eguale a 64 volte il cubo del raggio del cerchio circoscritto al triangolo ${ }^{3 \mathrm{r}}$ ). Dalla notissima formola di Eulero sulla curvatura delle superficie, il Mannheim concluse che : "la somma dei raggi di curvatura dei contorni apparenti di una superficie su due piani perpendicolari fra loro e ad un piano tangente fisso della stessa, non varia mutando comunque i due piani di projezione",; e siccome egli non aveva una sola corda al proprio arco, cosi segnaló un bel teorema da cui il precedente scaturisce senza il sussidio della polarità. - Dodici anni dopo, in seguito ad altre ricerche, di cui ci dovremo occupare altrove, il Mannheim investigò [II7] la figura che nasce polarizzando il sistema delle normali ad una superficie. - Finalmente, negli ultimi anni della sua vita, si servi della polarità, con l'ábilità consueta, per ottenere $[180]^{32}$ ) nuovi teoremi sulle normali alle

29) Eccone l'enunciato: Da un punto O preso nel piano d'una curva algebrica si conducano le tangenti a questa; si divida il raggio di curvatura corrispondente ad ogni punto di contatto per la sua distanza da $O$; la somma di tutti questi rapporti è eguale a o. [Nouvelles Annales de Mathématiques, IIème serie, t. IV (1865), p. 430, Question 745].

$\left.3^{\circ}\right)$ Vedi anche: Nouvelles Annales de Mathématiques, IIème série, t. I (I862), p. 29, Question 605.

$\left.{ }^{3} \mathbf{I}\right)$ Riguardo a queste due proposizioni, cfr. un reclamo di priorità fatto dal MannheIm nel L'Intermédiaire des Mathématiciens, t. XI (I904), p. 64. Un'altra proprietà di curvatura da lui avvertita nelle curve di terza classe trovasi nella Question 757 delle Nouvelles Annales de Mathématiques, IIème serie, t. V (1866), pp. I90-19г.

$\left.3^{2}\right)$ Tale Nota fu provocata da quella pubblicata nel i 877 dal Laguerre: Sur les normales que l'on peut mener d'un point donné à une conique [Comptes rendus hebdomadaires des séances de l'Académie des Sciences (Paris), t. LXXXIV (1er semestre 1877), pp. I81-I83.-Curres de LAGUerRe, t. II (Géométrie) (Paris, 1905), pp. 452-455]. 
coniche ${ }^{33}$ ) e per giungere a nuove proprietà focali delle stesse curve [192]; inoltre segnalo ${ }^{{ }^{3}}$ ) come questione importante da risolvere la ricerca dei teoremi che nascono applicando la stessa trasformazione alle proposizioni concernenti gli archí delle curve piane.

\section{Trasformazione per raggi vettori reciproci (inversione).}

La superficie ( ciclide") inviluppata da una sfera, che muovesi nello spazio conservandosi costantemente tangente a tre sfere fisse, venne incontrata da C. DupIN ${ }^{\mathbf{3 5}}$ ) come superficie avente per linee di curvatura delle circonferenze; una ciclide speciale, perchè è di rotazione (è l'ordinario "toro »), corrisponde all'ipotesi che i centri delle tre sfere date stiano in linea retta (cfr. [37]). Ora il Mannheim ha per primo osservato [I8] (cfr. [25]) che da qualunque ciclide nasce un toro applicandovi un'inversione il cui polo appartenga alla circonferenza determinata dai centri delle date sfere, e di tale osservazione trasse profitto per dedurre da un noto teorema del VILlarCEAU ${ }^{36}$ ) che "ogni sfera bitangente ad una ciclide la taglia secondo due circoli" e per dimosttare che "la ciclide è suscettibile di due generazioni come inviluppo di una sfera tangente a tre sfere fisse ». Siffatte considerazioni guidarono pure il MannHErm a molte proprietà, non tutte anteriormente note, delle superficie aventi per linee di curvatura d'un sistema delle curve sferiche, e ad un procedimento, che, se non c'inganniamo, non consegui tutta la meritata notorietä, per dedurre da una tale superficie un'altra della stessa specie.

Al Mannheim viene pure attribuita ${ }^{{ }^{3}}$ ) la notissima riduzione mediante inversione del problema di Apollonio alla ricerca di un cerchio tangente ad un circolo e due rette. A lui poi appartiene [23] (cfr. [35]) l'idea di considerare le curve e le superficie trasformate in sè stesse da un'inversione, idea che, nelle mani del Moutard, guidó alla teoria delle curve e superficie anallagmatiche e che $\mathrm{i}$ moderni estesero a tutte le trasformazioni geometriche. Non meno originale è l'applicazione [24] fatta dal Mannhem dell'inversione

33) Limitiamoci a rilevare la seguente bella osservazione di partenza: Una conica e la sua polare reciproca rispetto ad un cerchio di centro $O$ banno comuni le normali uscenti $d a O$.

34) Nel L'Intermédiaire des Mathématiciens, t. VI (I899), p. 4, Question 1430.

35) HACHETTE, Sur le contact des spbères; sur la sphère tangente à quatre sphères donnés; sur le cercle tangent à trois cercles donnés [Correspondance sur l'École Impériale Polytechnique, $t .1, z^{\mathrm{e}}$ édition (I8I3), pp. 17-28], p. 22 ; DupIN, Mémoire sur la sphìre tangente à trois ou à quatre autres [Ibid., t. II (1814), pp. 420-425]; Dupin, Applications de Géométrie et de Mécanique (Paris, 1822), p. 200.

${ }^{36}$ ) Questo teorema venne dimostrato piủ tardi [216] assai semplicemente dal nostro geometra; al quale appartiene anche una dimostrazione [215] della proprietà della terza sezione circolare del toro di tagliare tutti $\mathrm{i}$ meridiani secondo un angolo costante, proprieta enunciata, per la prima volta, dal colonnello Schclcher \{Théorie générale des bélices (riassunto) [Association Française pour l'Avancement des Sciences, Compte rendu de la XX ${ }^{\mathrm{e}}$ Session (Congrès de Marseille, I8gI), İ́re Partie, pagina 160$]$ \} e della quale, posteriormente, ebbe ad occuparsi G. Holzmoller \{Elementares über die Dupin'schen Cykliden und die Grundlagen der Krümmungstheorie [Zeitschrift für Mathematik und Physik, Bd. XLIV (I899), pp. I94-2 I3]';.

37) Nouvelles Annales de Mathématiques, Ière serie, t. XX (I86I), pp. 70-7I.

Rexd. Circ. Matem. Palermo, t. XXVI ( $2^{\circ} \mathrm{sem}$. 1908). - Stampato il 20 febbrajo 1908. 
alla teoria delle caustiche; è peró da lamentare che il relativo lavoro, contenendo soli enunciati, per la sua estrema concisione sia riuscito intelligibile soltanto a pochi eletti. $\dot{E}$ anche di pertinenza del Mannheis la prima dimostrazione geometrica [55] del bel teorema di Liouville, secondo cui il prodotto di quantesivogliano inversioni è un'inversione; essa è cosi semplice da essere alla portata anche di chi conosce soltanto la geometria di EucLIDE.

Altre applicazioni dell'inversione piana s'incontrano in uno degli ultimi lavori del ManNheim [I77 ${ }^{38}$ ), nel quale egli, servendosi per l'appunto di tale trasformazione, riusci a collegare fra loro alcune relazioni segmentarie dovute a STEwart e Chasles, e ad aggiungerne altre congeneri non meno eleganti. Della medesima trasformazione si parla in una questione che il Mannheim propose nel I904 sotto il pseudonimo di “ CANON » ${ }^{39}$ ), nella quale è segnalata una funzione metrica dell'insieme di due circonferenze, invariante rispetto a qualunque inversione ${ }^{4^{0}}$ ). Notiamo da ultimo che, quasi mezzo secolo $\mathrm{fa}^{4^{\mathrm{I}}}$ ), egli ha incidentalmente considerata la trasformazione che muta un punto in un cerchio ed una retta in un punto, trasformazione la quale si preferisce oggi di considerare come prodotto di una polarita per un'inversione.

\section{Geometria cinematica.}

E noto che la considerazione del centro d'istantanea rotazione piana condusse Chasles ad un metodo generale di costruzione delle normali alle trajettorie descritte dai singoli punti d'una figura piana moventesi comunque nel proprio piano. Ora Mannherm si propose la bella questione di estendere tale risultato allo spazio; e, giovandosi del moto elicoidale a cui equivale qualunque movimento infinitesimo di un solido, nonchè (per usare il linguaggio moderno) del complesso lineare connesso a tale movimento (l'uno e l'altro assai noti in Francia sin dal I 843 per merito di Chasces), pervenne [4I] ad un risultato a cui con ragione impose il nome di teorema fondamentale e che suona cosi: "Se in un istante qualunque si conducono le normali nei punti corrispondenti delle trajettorie dei vari punti di una figura invariabile parallele ad un piano fisso, si ottengono infinite relte che incontrano una seconda retta parallela a quel piano ». L'utilità di tale proposizione venne subito dal nostro geometra dimostrata applicandola allo studio del movimento d'un triedro di grandezza costante. Generalizzando quella proposizione si vede che, "quando un solido si muove nello spazio, le normali alle trajettorie dei suoi punti che incontrano una retta fissa ne tagliano anche una seconda".

$\left.{ }^{38}\right)$ Cfr. anche alcune osservazioni inserite nella Note del LAISANT relative aux asymptotes et aux cercles de courbure [Bulletin de la Société Mathématiqui de France, t. XXIII (1895), pp. 95-97], pp. 96-97.

39) L'Intermédiaire des Mathématiciens, t. XI (I904), p. 9.

$\left.4^{\circ}\right)$ E il rapporto delle lunghezze delle tangenti interna ed esterna di due circonferenze. L'invarianza segnalata dal MANNHEIM riesce evidente osservando che detto rapporto è una funzione dell'angolo sotto cui tagliansi $i$ due cerchi.

${ }^{41}$ ) Troisieme solution de la Question 273 [Nouvelles Annales de Mathématiques, Ière série, t. XIX (I860), Pp. IIj-II7], Pp. II6-II7. 
Donde un mezzo semplice per costruire il piano normale alla trajettoria d'un punto qualunque $M$ di un solido il cui movimento è regolato da cinque condizioni : cosi, ad es., se cinque punti del solido sono costretti a rimanere su altrettante superficie date, in un istante qualunque le normali in quei punti a tali superficie determinano un complesso lineare ed il cercato piano normale è quello che corrisponde a $M$ rispetto a tale complesso ${ }^{42}$ ). Se invece il solido non è soggetto che a quattro condizioni, i suoi punti descrivono, non delle linee, ma delle superficie-trajettorie ed in un istante qualunque le normali in punti corrispondenti s'appoggiano a due rette fisse ${ }^{43}$ ); proprietà questa straordinariamente importante, che il nostro geometra comunico alla Società filomatica di Parigi il I4 luglio i $866^{44}$ ), ma la cui prioritd gli venne contestata dallo Schönemann, che sin dal 1855 avevala dimostrata in una memoria presentata da STEIner all'Accademia delle Scienze di Berlino ${ }^{45}$ ): l'indipendenza delle due scoperte essendo fuor di dubbio è pienamente legittimo il nome di teorema di Schönemann-Mannheim dato a quella proposizione ${ }^{46}$ ).

La grande fecondità dei germi consegnati nel lavoro [4I] ora analizzato risulta palese esaminando un'altra memoria [48] di poco posteriore, la quale, presentata all'Istituto di Francia [46], fu oggetto di una lusinghiera relazione firmata da Bertrand, Bonnet e Chasles, ben meritato compenso alle lunghe fatiche durate dal Mannheim; una memoria riboccante di cose nuove ed interessanti, ed il cui stile preciso ed elegante prova la padronanza del soggetto da parte dell'autore e la maturità da questi ormai raggiunta. Esordendo egli cita alcune belle frasi scritte da Pornsot nella sua celebre Théorie nouvelle de la rotation des corps e destinate a mettere in luce $\mathrm{i}$ vantaggi del metodo geometrico nello studio delle questioni dinamiche. Dimostra poi con metodo uniforme le proposizioni fondamentali di cinematica enunciate da CHASLEs in una comunicazione fatta nel 1843 all'Accademia delle Scienze. Sei condizioni essendo necessarie e sufficienti per fissare la posizione di un solido nello spazio, ove non se ne abbiano che cinque o quattro, ogni punto del solido potrà assumere $\infty^{1} \mathrm{o} \infty^{2}$ posizioni, onde

$4^{42}$ ) I particolari della costruzione di detto piano si leggono in una Questione ( $\mathrm{n}^{\circ} 806$ ) proposta dal Mannheim nelle Nouvelles Annales de Mathématiques [II série, t. VI (I867), p. I88].

43) Queste rette sono in generale sghembe; se per eccezione si tagliano, tutte quelle normali passano per un punto fisso: per quali movinenti cio accade ? E la Questione $\mathrm{n}^{0} 553$ proposta dal Mannheim nel L'Intermédiaire des Mathématiciens [t. II (I895), p. I62] e non ancora risoluta.

44) La relativa comunicazione (Sur le déplacement d'un corps solide) trovasi riprodotta in seguito della Memoria [4I].

45) Dietro consiglio del Prof. GeIser tale Memoria (Ueber die Construction von Normalen und Normalebenen gewisser krummer Flächen und Linien) venne riprodotta nel tomo XC (I88I), pp. 44-48, del Journal für die reine und angewandte Mathematik, affinchè conseguisse la notorietà che erale stata sino allora negata.

${ }^{46}$ ) In virtù di essa la costruzione della normale in un punto qualunque d'una superficie trajettoria è ricondotta a quella di una retta che passa per un punto e si appoggia a due rette sghembe: nel caso in cui queste si ano immaginarie coniugate, per eseguire realmente tale costruzione fa mestieri ricorrere ad artifici che il MANNHEIM ha suggeriti altrove [118]. 
descrivera una trajettoria lineare o superficiale. Ora il Mannherm prova che, se i vincoli del movimento consistono in passaggi o contatti di curve e superficie per punti dati o con linee o superficie fisse, quando si tratti di movimenti infinitesimi le condizioni date possono sempre immaginarsi consistere in passaggi per dati punti di superficie appartenenti al sistema mobile; è cosi condotto allo studio del moto di un solido di cui cinque superficie assegnate passano per altrettanti punti fissi e lo compie nel modo più luminoso e completo, ottenendo una folla di teoremi la cui novità è pari all'importanza. Nella $2^{\text {a }}$ Parte del suo lavoro il nostro autore applica la teoria generale esposta al movimento di una retta (caso di grande interesse per la teoria delle superficie rigate), di una coppia di piani fra loro invariabilmente legati, di un triedro (con speciale riguardo al caso in cui gli spigoli di esso siano la tangente, la binormale e la normale principale d'una curva gobba) o di una superficie. Due cose vanno ancora notate; cioè che gli è nel corso di tali ricerche che il Mannherm venne condotto ad introdurre ( $\mathrm{p} .98$ ) la nozione di normalia (superficie costituita dalle normali condotte ad una superficie dai varî punti d'una sua linea) e che i cultori della geometria descrittiva troveranno al termine della memoria testè analizzata alcune notevoli costruzioni concernenti l'elicoide rigata.

Come saggi di ulteriori studi del Mannheim sopra questioni cinematiche sono da considerarsi due comunicazioni $[5 \mathrm{I}],[52]$ da lui fatte all'Istituto di Francia, le quali contengono i soli enunciati di teoremi scoperti e problemi risolti applicando alcune formole allora inedite e che tali dovevano rimanere per lungo tempo ${ }^{47}$ ).

In seguito egli hạ osservato [56] che, quando il moto di un solido è soggetto a quattro sole condizioni, gli assi di tutti gli spostamenti che esso puó subire a partire da una posizione qualunque costituiscono una speciale conoide del terz'ordine: è la superficie scoperta da HamiLTon ( 830 ) e impropriamente chiamata il cilindroide di Cayley a conoide di PlÜCKER, di cui è noto l'intervento in svariate questioni geometriche e meccaniche e di cui il Mannheim si occupó, a varie riprese, per tutta la sua vita ${ }^{4^{8}}$ ).

Il movimento d'un solido soggetto a sole quattro condizioni, del quale il Mannheim ha la gloria di avere iniziato lo studio, forma il tema anche di un altro suo esteso lavoro [9I], nel quale giova rilevare anzitutto l'osservazione (cfr. [7I]) nuova, ed allora di apparenza paradossale, che in qualunque movimento vi sono degli elementi fissi: sono i punti ciclici, se si tratta d'un moto piano, è il circolo immaginario all'infinito,

\section{7) Cfr. [184], pp. 457-475.}

$4^{8}$ ) Cosi egli ha notato [159] che tale superficie puó generarsi mediante un'ellisse di forma e grandezza variabili di cui gli estremi dell'asse minore incontrano due spigoli di un triedro trirettangolo e segante costantemente il terzo spigolo. Più tardi egli stabili geometricamente [I87] che, come aveva notato F. Michel, se si applica ad una conoide di PuUcker un'inversione col polo sull'asse della sup'ritis, si ottiene quella supsrficie di quart'ordine con retta doppia che é il luogo dei cerchi di curvatura in un punto di una superficie delle sezioni prodotte nella stessa da piani per quel punto. Finalmente ha proposto [L'Intermédiaire des Mathématiciens; t. XII (Igos), p. 5] il problema di definire la superficie in questione, non come luogo di rette od ellissi, ma come luogo di punti. Cfr. anche [152], pp. 435-44I. 
se si tratta del moto d'un solido. Penetrando poi nell'anima del suo tema, il Mannheim dimostra essere un'iperboloide il luogo delle normali alle superficie trajettorie de' punti d'una retta qualunque dello spazio e ne trae una puova dimostrazione (cfr. [72]) dell'esistenza di due rette $D, \Delta$ incontrate dalle normali alle superficie trajettorie di tutti i punti di una figura di forma invariabile mobile. L'artificio qui usato di dedurre le proprietà relative al moto d'una figura qualsiasi dalle analoghe concernenti una retta, viene dal nostro autore applicato più e più volte, assurge quindi alla dignità di metodo e mena a conseguenze interessantissime. Lo spazio non concedendoci di riferirle per esteso, ci limitiamo ad enunciare le questioni a cui esse si riferiscono: Di qual ordine è il luogo dei punti d'un solido le cui trajettorie : a) sono tangenti alle linee asintotiche delle superficie trajettorie dei punti stessi; $b$ ) osculano le geodetiche delle superficie trajettorie; c) hanno raggio di curvatura nullo; $d$ ) sono tangenti alle linee di curvatura delle superficie trajettorie; $e$ ) hanno un raggio di curvatura principale infinito; $f$ ) hanno i loro raggi di curvatura eguali e dello stesso segno oppure di segni contrari? Dalle soluzioni si desume l'esistenza di parecchie curve e superficie algebriche collegate a qualsiasi movimento di una figura solida: "Les géométres comprendront, sans que nous ayons besoin d'insister, toute l'importance d'un travail qui réunit dans une théorie absolument nouvelle, en les déduisant d'un mode uniforme de démonstration, des résultats aussi précis et aussi considérables » ${ }^{49}$ ).

Nella chiusa di tale memoria il Mannherm allude a speciali movimenti pei quali non sussistono tutte le proprieta esposte, mentre altre se ne presentano. Ora, ad un notevole moto particolare d'un solido qualsivoglia egli consacrò più tardi un importante lavoro $[\mathbf{I} 73]^{50}$ ), nel quale è risoluta la seguente questione: Determinare direttamente le condizioni affinchè il movimento d'un solido sia tale che qualunque piano ad esso collegato passi per un punto fisso. In tali ipotesi, come osservò il Darboux ${ }^{5 \mathbf{r}}$ ), quei piani inviluppano ciascuno un cono di rotazione; MannheIM aggiunse che gli assi di tali coni sono fra loro paralleli e che al mobile è collegato un fascio di piani i cui singoli elementi passano per altrettante rette fisse, generatrici d'un cilindro. Il detto movimento, se è possibile, puó intendersi ottenuto, vuoi facendo muovere un triedro rigido in modo che due facce passino ciascuna per una retta fissa e la terza per un punto fisso, vuoi combinando il ruzzolamento di un cilindro rotondo sopra un altro di raggio metà con una traslazione parallela alla comune direzione degli assi di tali cilindri. Tutte queste proposizioni postulano la possibilità del movimento in questione; per dimostrarla, l'autore,

49) Queste parole di Chastes si trovano in una bellissima Relazione, letța all'Accademia delle Scienze di Parigi il 6 ottobre 1873 (a nome di una Commissione di cui gli altri membri erario BerTrand e Bonnet) [Comptes rendus hebdomadaires des séances de l'Académie des Sciences (Paris), t. LXXVII $\left(2^{\mathrm{e}}\right.$ semestre $\left.\mathrm{I} 873\right)$, pp. $752-75^{6}$ ], per effetto della quale il lavoro in questione venne pubblicato nel tomo XXII dei Mémoires présentés par divers savants à l'Académie des Sciences (Paris).

$\left.5^{\circ}\right)$ Vedi anche [178]. Cfr. [184], pp. 389-399.

${ }^{5}$ ) Sur le déplacement d'une figure invariable [Comptes rendus hebdomadaires des séances de l'Académie des Sciences (Paris), t. XCII (Ier semestre I88I), pp. II8-I2I]. 
servendosi di uno speciale moto piano, fa vedere che quando un triedro rigido si muove in modo che due sue facce siano costantemente tangenti a due coni rotondi ad assi paralleli mentre la terza passa per un punto fisso, qualsivoglia piano connesso al triedro passerà per un punto fisso. "La démonstration de l'existence d'un pareil déplacement », osserva finendo il nostro geometra, " ne permet plus de mettre en doute sa possibilité, mais elle ne détruit pas cependant un sentiment d'étonnement que légitime son impossibilité apparente "; perciò sarebbe desiderabile ed utile qualche apparato che servisse ad illustrarne tutte le circostanze ${ }^{\mathbf{5 2}}$ ).

Il più semplice sistema di forma invariabile è la retta; il movimento di essa (del quale, come vedemmo, il Mannheim erasi incidentalmente occupato in precedenti pubblicazioni) diede materia ad un lavoro ([68] ossia [69] e [7o]) ${ }^{53}$ ), dal quale togliamo i seguenti enunciati: Le tangenti alle trajettorie dei singoli punti di una retta in un istante qualunque costituiscono un parabolvide iperbolico, mentre $i$ relativi piani osculatori inviluppano una superfici di quart'ordine e tera a classe; sulla retta mobile in generale non esiste alcun punto che sia flesso della corrispondente trajettoria, ma, se ve ne è uno, i piani osculatori in punti corrispondenti delle trajettorie di tutti $i$ punti della retta mobile inviluppano un cilindro; gli assi di curvatura (vedi più avanti) in punti corrispondenti delle trajettorie dei punti di una retta mobile sono generatrici di una quadrica ${ }^{54}$ ); ecc. Fra le molteplici conseguenze di queste proposizioni limitiamoci a notare la seguente: Se quattro punti d'una retta mobile descrivono ciascuno un piano, un quinto punto qualsiasi di essa descriverà una conica ${ }^{55}$ ); le infinite coniche analoghe banno $i$ loro centri su di una retta. La riferiamo non solo perchè ci sembra notevole e perchè servi di punto di partenza per importanti indagini dell'Halphes ${ }^{56}$ ), ma perchè la si ritrova in un posteriore lavoro $\left[\mathrm{I} 6_{4}\right]^{{ }^{5}}$ ), nel quale il nostro scienziato stabili per via esclusivamente geometrica elementare tutte le circostanze di un movimento tale che tutti i punti della figura mobile descrivano delle ellissi, giungendo a dimostrare che lo si puó ottenere combinando il moto di ruz-

${ }^{52}$ ) Della costruzione di tale apparato si occupò il Prof. A. GRunwald: v. la recente memoria Darstellung der MANNHEIM-DARBOUX'schen Umschwungsbewegung eines starren Körpers [Zeitschrift für Mathematik und Physik, t. LIV (1907), pp. [54-220].

53) In un altro [73] egli dimostrò che ai teoremi relativi alle superficie trajettorie dei punti d'una retta si possono farne corrispondere altrettanti relativi alle superficie inviluppate dai piani d'un fascio rigido (cfr. anche [179]). Aggiungiamo qui che della cinematica di un fascio rigido tratta anche una posteriore memoria $[8 \mathrm{I}]$, che siamo forzati a semplicemente ricordare.

54) Che tale superficie non sia affatto generale sembra risultare dalla seguente questione indarno proposta da Mannheim: In qual modo riconoscere se le generatrici d'uno stesso sistema d'un'iperboloide possono essere gli assi di curvatura delle trajettorie dei punti di una retta mobile? [L'Intermédiaire des Mathématiciens, t. II (I895), p. I62, Question 554].

55) "Quali sono le relazioni esistenti tra gli archi di tali linee simultaneamente descritti ? ) chiese (e sinora invano) il Mannheim. [L'Intermédiaire des Mathímaticiens, t. II (I895), p. I62, Question 552 ].

${ }^{56}$ ) Sur le mouvement d'une droite [Bulletin de la Société Mathématique de France, t. I (1873), pp. II4-II7].

57) Cfr. [184], pp. I69-176. 
zolamento di un cilindro circolate retto sopra un altro di raggio doppio, con una traslazione parallela all'asse del cilindro.

Al movimento di una retta si riferisce pure un lavoro del Mannherm di più antica data [I48], il cui precipuo intento é di stabilire una bella proposizione contenuta nella Nota del Darboux: Une nouvelle définition de la surface des ondes ${ }^{58}$ ) ed alcuni complementi dativi dallo stesso geometra in un corso professato alla Sorbona. Il Mannheim suppose, col Darboux, che una retta $D$ muovasi nello spazio con la condizione che tre suoi punti descrivano le facce d'un triedro trirettangolo e dimostro che essa è costantemente normale ad una (quindi ad $\infty^{1}$ ) superficie suscettibili d'un'elegante definizione geometrica; incidentalmente ottenne alcune costruzioni e proposizioni bellissime che ci duole di non potere riferire.

Analogo a tale movimento è quello di una retta $D$ [I68], [I69]; [I 7o], regolato dalla condizione che tre suoi punti $a, b, c$ percorrano altrettante sfere coi centri $\alpha, \xi, \gamma$ sopra una retta $O$ : allora qualunque altro punto $l$ di $D$ percorre pure una sfera col centro $\lambda$ in $O$; e se s'immagina che una fila di sfere coi loro centri in $D$ formino una figura rigida mobile con la condizione che tre suoi elementi tocchino altrettante sfere fisse coi centri in punti di $O$, qualunque altra sfera della fila toccherà sempre un'altra analoga sfera. In particolare, se $D$ trovasi all'infinito, quelle sfere divengono piani paralleli ad una retta fissa e si conclude che, se tre piani di un fascio rigido si muovono toccando costantemente tre sfere coi centri sopra una retta $O$, qualunque altro piano del fascio toccherà un'analoga sfera. Da questa curiosa considerazione scaturisce un procedimento per dedurre da ogni proposizione concernente la cinematica di una retta un teorema congenete relativo ad un fascio di piani, passando attraverso ad uno relativo ad una fila di sfere. Nell'impossibilità di enumerare tutte le applicazioni fattene dal Mannherm, ci basti segnalare la dimostrazione da lui data [r79] per l'identità sostanziale delle due seguenti proposizioni :

Se quattro punti di una retta percorrono altrettante sfere coi centri in un piano, ogni altro punto di quella retta descriverà una sfera analoga.

Se quattro piani d'un fascio rigido toccano altrettante sfere coi centri in un piano, ogni altro piano di quel fascio toccherà una sfera analoga.

Un'altra figura speciale per semplicità paragonabile alla retta è il diedro rigido; il Mannheim ne investigó il moto infinitesimo [IOI], dimostrando che in generale esso equivale ad una semplice rotazione; ma se, mentre lo spigolo del diedro genera un elemento di superficie gobba, una delle facce ha per caratteristica una retta perpendicolare allo spigolo stesso, lo stesso accadrd per l'altra faccia e quel moto infinitesimo non equivarrà che ad una semplice traslazione (cfr. [ I I 4]). L'importanza geometrica di questo risultato è dimostrata dall'avere il Mannheim ottenuto col suo mezzo [I 42] l'estensione

$5^{\mathbf{8}}$ ) Comptes rendus hebdomadaires des séances de l'Académie des Sciences (Paris), t. XCII (1881), pp. $446-448$. 
allo spazio del teorema di RÉAuniur che sta a base della teoria delle evolute oblique ${ }^{59}$ ); ci sia lecito riferime l'enunciato: $\dot{E}$ data una superficie sviluppabile; un diedro rigido si muove con la condizione che il suo spigolo ne sia sempre una generatrice e che una sua faccia ne sia sempre un piano tangente; in conseguenza l'altra faccia genera una seconda superficie sviluppabile, di cui le singole generatrici sono le projezioni ortogonali, sopra le seconde facce del diedro mobile, degli assi di curvatura della data superficie.

Ad applicare $\mathrm{i}$ concetti ed i metodi della Geometria cinematica a figure più complicate delle punteggiate e dei fasci di piani il Nostro $f u$ indotto dalle note ricerche che condussero il Visconte de Sparri a rettificare un punto essenziale della Théorie nouvelle de la rotation d'un corps del Pornsot ${ }^{\circ}$ ). Studiando direttamente ed esaurientemente [I 45], [I 46] il moto d'un ellissoide col centro fisso che ruzzola sopra un piano fisso, egli giunse nel modo più spontaneo alle conclusioni del DE SPArre e ad altre che vi si connettono.

Sopra siffatte questioni egli è ritornato [I S3], [I 54], [I S5], [1 56] in seguito agli studi iniziati da CAYLeY ${ }^{\text {or }}$ ) e LARMOR ${ }^{62}$ ) relativi all'iperboloide rigato deformabile, provocati da un bel quesito proposto dal Greenhill il I 4 Gennajo I 878 alla "Senate-House Examination s ${ }^{63}$ ) e proseguiti brillantemente in Francia dal Darboux ${ }^{64}$ ); applicandovi i procedimenti caratteristici della Geometria cinematica egli riusci a collegare armonicamente i risultati del DE SPARRE ai celebri teoremi di Ivory sulle quadriche omofocali ${ }^{65}$ ).

Ricordiamo anche, prima di volgerci ad altro soggetto, le ricerche fatte dal MaNNHELM, in seguito ad una comunicazione del RESAL ${ }^{66}$ ), sul movimento d'un doppio

59) Cfr. G. LorIA, Spezielle algebraische und transzendente ebene Kürven, Theorie und Geschicbte (Leipzig I902), p. 628.

6o) De SPARre, Sur l'erpolodie de Poinsot [Comptes rendus hebdomadaires des séances de l'A. cadémie des Sciences, t. XCIX ( $2^{\mathrm{e}}$ semestre I884), pp. 906-909].

6I) The Messenger of Mathematics, Series II, Vol. VIII (1879), pp. 51-52; The collected Papers, Vol. XI (1896), pp. 66-67.

62) Proceedings of the Cambridge Philosophical Society, Vol. V (I884), pp. I6I-I67.

63) Eccone lo storico enunciato: Dimostrare che se un iperboloide ad una falda è costiluito da aste articolate nei loro punti d'intersezione, deformandolo si ottiene un iperboloide omofocale al primilivo, ogni punto della superficie descrivendo una trajeltoria ortogonale di tale sistema di iperboloidi.

64) sur la thícrie de PoINsor et sur deux mousements correspondant à la mime polbodie [Comptes rendus hebdomadaires des séances de l'Académie des Sciences (Paris), t. C (i ${ }^{\text {er }}$ semestre 1885 ), pp. 155j-156I]; Sur le mouvement d'un corps pesant de révolution, fixé par un point de son axe [Ibid., t. CI ( $2^{\mathrm{e}}$ semestre 1885$)$, pp. II-17, II 5-I 19]; Sur diverses propositions relatives au mouvement d'un corps solide autour d'un point fixe [Ibid., t. Cl (2 $2^{\mathrm{e}}$ semestre 1885$)$, pp. 199-205].

65) $\mathrm{Da}$ queste ricerche sull'iperboloide articolato il MA.NnHerm fu condotto [163] alla seguente generazione dell'ellisse: Due segmenti o $p, o q$ di lunghezze costunti ruotuno in un piano attorno ad o di angoli eguali ed in sensi opposti; il luogo geometrico del centro $m$ del segmento $p q \dot{e}$ un'ellisse $i$ cui assi sono in direzione le bisettrici dell'angolo poq ed in grandezza op \pm oq. Tale teorema (denominato " teorema di Mannherm » a pag. 507 del Cours de Géométrie élémentaire par F. G. M.) venne dal Nostro applicato alla ricerca del centro di curvatura dell'evoluta dell'ellisse.

66) Ėtude du mouvement d'un double cône paraissant remonter, quoique descendant, sur un plan incliné [Comptes rendus hebdomadaires des séances de l'Académie des Sciences (Paris), t. CXI ( $2^{\mathbf{e}}$ semestre I 890), pp. 547.553]. 
cono vincolato da varie condizioni [ $\mathrm{I}$ II], [I72] ed i notevoli contributi da lui dati [200] alle indagini di R. BrICARD ${ }^{67}$ ) sull'ottaedro articolato; e giova rilevare come ivi gli aspiranti a proseguire nella via spianata dal nostro autore leggeranno gli enunciati di tutta una serie di questioni importanti e tuttora irresolute concernenti il movimento d'un triangolo rigido.

Chiuderemo questa rassegna dei lavori cinematici del Mannherm segnalando le dimostrazioni elementarissime da lui date [I50], [205] per le proprietà caratteristiche dei noti sistemi articolati dell'Hart e del KEMPE ${ }^{53}$ ) e $\left[90{ }^{69}\right.$ ) per le due seguenti elegantissime proposizioni (di cui la prima appartiene a Sylvester), entrambe collegate alla teoria degli « inversori »:

I. Se $a b c d$ è un quadrilatero articolato tale che $a b=a d, b c=c d$ e se lo si deforma tenendo fissi $i$ vertici $a, d$, un punto $m$ invariabilmente connesso al lato $b c$ descriverà una podaria di conica.

II. Quando un quadrilatero articolato scaleno agmd ba le diagonali ag, md fra loro perpendicolari e lo si deforma in modo che il vertice a descriva una circonferenza $e$ che $i$ vertici $g$, $d$ descrivano due circonferenze aventi per comune centro un punto di ma, il vertice libero $m$ descriverà una quartica anallagmatica.

\section{Geometria infinitesimale.}

Mentre la geometria infinitesimale trattata col sussidio delle coordinate è intimamente legata al formalismo leibniziano del calcolo sublime, la trattazione sintetica di quel nobilissimo ramo dello scibile matematico è, e sembra dovere sempre rimanere, indissolubilmente connessa alla concezione newtoniana del calcolo stesso; ché l'investigazione delle qualità più riposte delle curve e delle superficie si direbbe non potere essere effettuata geometricamente che da chi ne studia la genesi per mezzo di enti più semplici (punti, rette, piani) variabili con legge determinata. Niuna meraviglia, pertanto, se $i$ lavori di cui ora imprendiamo l'analisi siano cosi strettamente avvinti a quelli dianzi discorsi che il separarneli, come noi facciamo, sia da ritenersi per un semplice artificio espositivo analogo a quello che fa considerare separatamente causa da effetto, teoria da applicazione.

Geometria piana, - Una curva piana qualunque $\Gamma$ si può notoriamente rappresentare mediante un'equazione $f(r, s)=0$ fra il suo raggio di curvatura $r$ in un punto qualunque $P$ e la lunghezza $s$ del suo arco compreso fra $P$ ed un punto fisso di $\Gamma$ : è questo il sistema di coordinate che sta a base della Geometria intrinseca. Ora se s'interpretano $r, s$ come coordinate cartesiane ortogonali d'un punto del piano, l'equazione $f(r, s)=0$ rappresenta una curva 1 , tale che [come osservó il Mannheim [I $\left.s]^{7^{\circ}}\right)$ ]

${ }^{6}$ ) Mémoire sur la théorie de l'octaèdre articulé [Journal de Mathématiques pures et appliquées, $V^{e}$ série, t. III (1897), pp. II3-148]. Vedi in particolare, p. I34, nota.

63) Cfr. [I52], pp. 216-222.

69) Cfr. [184], pp. 6 e $7^{6-81}$.

7o) Cfr. [184], pp. 500-510.

Rend. Circ. Malem. Palermo, t. XXVI ( $2^{\circ}$ semestre 1908$)$. - Stampato il 21 febbraje 1908. 
se si fa ruzzolare $\Gamma$ sopra una retta $g$ e per ogni posizione si costruisce il centro di curvatura di $\Gamma$ nel corrispondente punto di contatto, il luogo dei punti risultanti, rispetto ad un sistema cartesiano di cui $g$ sia un asse, ha appunto per equazione $f(r, s)=0$. Il Mannheim ha insegnato a costruirne la tangente in un punto qualunque ed ottenerne per via geometrica la rettificazione e la quadratura, ed ha fatto vedere come essa guidasse alla scoperta di eleganti proprietà ${ }^{71}$ ) di parecchie curve speciali (spirale logaritmica, curve cicliche, catenaria e [97] spirali sinusoidi). L'originalitì e fecondità di siffatta considerazione consigliarono E. WöLfFing ${ }^{\prime 2}$ ) a chiamare $\perp$ la curva di Mannheim della $\Gamma$, proposta assennatissima che venne generalmente accettata ${ }^{73}$ ).

Non sono questi gli unici frutti che il Nostro abbia raccolti dal considerare il moto di ruzzolamento di una curva sopra una retta. Infatti ${ }^{74}$ ) E. Catalan, appunto per incarico del suo antico discepolo, comunico alla Societa filomatica di Parigi la seguente proposizione: Quando una curva piana I ruzzola sopra una retta fissa, un punto qualunque $M$ collegato a $\Gamma$ descrive una curva di lungbezza eguale alla podaria di $M$ rispetto a $\Gamma$ stessa. Pubblicata nel giornale "L'Institut" del 25 febbrajo 1858 , assieme a svariate conseguenze, attrasse subito l'attenzione d'un altro eminente cultore della geometria cinematico-infinitesimale, il LAMARLE, il quale si affrettó a dimostrarla e generalizzarla ${ }^{7^{5}}$ ). D'altronde essa venne incontrata, circa nello. stesso tempo, da P. Serret ${ }^{76}$ ) e prima di tutti era stata scoperta da STEINER ${ }^{77}$ ), nel corso delle sue famose ricerche sui baricentri di curvatura delle linee piane; onde il Mannherm, con l'onestà che lo distingueva nella vita scientifica come nella vita privata, propose designarla col nome di teorema di Steiner. Quali e quante notevoli generalizzazioni egli ne abbia date noi diremmo se la tirannia dello spazio non lo vietasse; non solo egli suppose che il punto $M$, che interviene nel relativo enunciato, fosse per proprio conto animato da un movimento qualunque, ma estese le proprie considerazioni alla sfera ed allo spazio. Le proposizioni ottenute concernono il paragone e le misure di archi di curve e chi volesse dimostrarle col sussidio dell'analisi dovrebbe riconoscere di trovarsi alle prese con un genere di questioni in cui questa è vinta dalla sua eterna rivale. $E$ va notato che l'edificio veramente grandioso eretto dal Mannherm poggia sopra una semplice formola, la quale dà, sotto forma finita, il rapporto dei cammini elementari descritti dagli estremi

$\mathbf{7 r}^{\mathbf{r}}$ Vedi Loria, loco citato 57), pp. 433, 47 I-472, 49I, 50I-502, 577.

72) Über Pseudotrocboiden [Zeitschrift für Mathematik und Physik, Bd. XLIV (1899), pp. 139-I66].

73) Cfr. H. WIELEITNER, Über eine Verallgemeinernng des Begriffes der MannHeIM'schen Kurve [Mathematisch-naturwissenschaftliche Mitteilungen (Stuttgart), Ser. II, Bd. IX, Heft I (März 1907), pp. I-9]. P. ERNST, Ein Analogon zur MannheIm'schen Kurve [Monatshefte für Mathematik und Physik, Bd. XVIII (I907), pp. 315-316].

74) Per ciò che segue vedi la memoria [30] riprodotta in [I84], pp. 5II-530.

75) Sur un théorème rélatif à la théorie des roulettes [Bulletins de la classe des Sciences de l'Académie Royale de Belgique, II ${ }^{\mathrm{e}}$ série, t. IV (I8;8), pp. 239-248].

$\left.{ }^{76}\right)$ Théorème sur les roulettes [Nouvelles Annales de Mathématiques, İ̀re série, t. XVIII (I 859 ), pp. 34I-342].

77) Gesammelte Werke, Bd. II (Berlin I882), p. IOI. 
d'una retta, formola che, mentre abilita a risolvere altre questioni ancor più generali, guida a nuove proprietà di curve spcciali.

Del resto non è questo il più antico contributo che egli abbia dato alla trattazione sintetica di elevate questioni di Calcolo integrale; giacchè prima la lettura del Théorème sur les arcs des lignes aplanétiques di W. RoBERTs ${ }^{78}$ ) avevalo condotto $\left[26{ }^{79}\right.$ ) ad osservare come una qualunque delle curve ivi considerate (cioè rappresentabile in coordinate polari $r, \omega$ mediante un'equazione del tipo $r^{2}-2 r \Omega+\alpha=0$, ove $\Omega$ è una data funzione di $\omega$ ) è l'anallagmatica inviluppata dagli $\infty^{1}$ cerchi ortogonali ad un cerchio fisso ed aventi i loro centri sopra l'antipodaria dell'origine rispetto alla curva $r=\boldsymbol{\Omega}$. Tale circostanza venne da lui sfruttata per trasformare e stabilire geometricamente alcune formole di rettificazione scoperte dal ROBERTs e dedurre una bellissima proprietd dallo stesso avvertita nelle ovali di Descartes. Si noti anche che, nello stesso lavoro, il MANNHEIm ha introdotta la considerazione di una nuova curva connessa ad una data curva $\Gamma$; è l'inviluppo I dei cerchi che hanno i loro centri su $\Gamma$ e per raggi i relativi raggi di curvatura, ed ha dimostrato che ogni arco di $\Gamma$ è uguale alla differenza dei due corrispondenti archi di I. Egli ha poi estese alla sfera le considerazioni testè compendiate e ne ha dedotta una bella proprietà dell'ellisse sferica, che, tenendo conto di una estensione posteriore (v. [3o], p. 228 nota), suona cosi: Da tutti i punti d'un'ellisse sferica come poli si descrivano dei circoli massimi i cui raggi sferici abbiano i seni proporzionali ai seni delle distanze dei loro poli da un fuoco di quell'ellisse: gli archi corrispondenti del loro inviluppo avranno differenze esprimibili mediante archi di circoli.

Considerazioni della stessa natura si ritrovano in altro lavoro [97 ${ }^{80}$ ) dedicato alle curve oggi chiamate spirali sinusoidi ed a quelle generate dal polo di una di esse, quando la spirale ruzzola sopra una retta; lavoro che, dovendo ascriversi alla categoria delle opere minori del Mannheim, non puó ricevere qui più di un cenno fugace.

A tali ricerche di Calcolo integrale sintetico ne fanno riscontro altre di Calcolo differenżale sintetico, le quali, benchè abbiano portata assai minore delle altre, non meritano di essere condannate all'ostracismo della nostra analisi.

Quelle di più antica data si connettono alle immortali proposizioni di Ponceler sopra i poligoni inscritti e circoscritti alle coniche e concernono gli $n$-goni semplici i cui lati toccano date curve e di cui $n$ - I vertici appartengono a linee assegnate; per determinare la tangente in un punto arbitrario del luogo del vertice libero d'un tale poligono, il Mannherm esumó una formola di Newton che leggesi nell'introduzione al Tractatus de quadratura curvarum ${ }^{8 \mathrm{r}}$ ) e ne dedusse due teoremi generali, la cui fecondità fu da lui stesso dimostrata traendone elegantissime nuove proprietà delle curve del $2^{\circ}$ e del $3^{\circ}$ ordine e risolvendo ${ }^{82}$ ) il seguente bel problema: Un poligono si muove

${ }^{78}$ ) Journal de Mathématiques pures et appliquées, Ière série, t. XV (1850), pp. I94-196.

79) Cfr. [184], pp. 8I-93.

80) Cfr. [184], pp. 530-532.

81) Cfr. [152], p. 2 10.

82) In una comunicazione fatta alla Società filomatica di Parigi il 5 maggio 1860. 
nel proprio piano conservandosi simile a sè stesso; si conoscono $i$ centri di curvatura, tanto della curva inviluppata da un suo lato, quanto delle curve descritte dagli estremi dello stesso; determinare $i$ centri di curvatura in un punto qualunque della linea descritta da un altro vertice od inviluppata da un altro lato del poligono.

Soltanto novità di metodo offre una breve Nota [29] in cui giungesi, per altra via, ad una costruzione che Poncele't aveva dedotta dal ben noto metodo delle tangenti del Roberval. Aggiungiamo che il Mannheim ritornó trentaquattro anni più tardi sulla medesima questione [208], per segnalare altre due costruzioni analoghe (una delle quali già da lui esposta [I 52], p. 38I), applicabili quando, per la disposizione dei dati, la prima cessa di esserlo; a tale proposito egli osserva che, i lavori geometrografici del Lemorne lasciando intatta la questione dell'applicabilità delle soluzioni, torna della massima importanza averne a propria disposizione parecchie, da usare a seconda delle circostanze.

In altro lavoro [3I] (che, benchè di aspetto modesto, ottenne il più lusinghiero giudizio da parte di Poncelet ${ }^{83}$ ), mediante un'ingegnosa applicazione del teorema di Carnot, il Mannheim giunge alla seguente proposizione nuova ed elegante: Siano $B$, $C$ due punti di una curva del terzordine, $A$ il punto in cui si tagliano le relative tangenti, $b$ il terzo punto comune alla curva ed alla retta $B C$, e $D$ il punto in cui questa retta è incontrata dalla congiungente delle terze intersezioni della data curva con le tangenti $A B, A C$; allora il rapporto dei raggi di curvatura di tale curva in $B, C$ è dato da

$$
\frac{\overline{A B}^{3} \cdot \overline{C D} \cdot \overline{B b}}{\overline{A C}^{3} \cdot \overline{B D} \cdot \overline{C b}}
$$

Quando la cubica si spezza in una retta ed una conica, si ricade in una proprieta delle coniche, di cui già parlammo nel Cap. prec.

Un più recente lavoro semianonimo [I 20] del Nostro, pur non avendo che il modesto scopo di mostrare sopra un esempio la natura e la fecondita della Geometria cinematica, contiene qualche utile osservazione sulle asteroidi ${ }^{34}$ ). In altro [ 166$]$ gli stessi metodi conducono ad una nuova soluzione di una questione già trattata dal LAISANT in una comunicazione fatta alla Societa matematica di Francia il i8 luglio i888. Essi permisero poi un esauriente studio [I6I] dell'inviluppo della congiungente due punti mobili su di un cerchio con velocità una doppia dell'altra, e diedero eleganti costruzioni per la tangente all'ovale di Cassini [I76], la normale alla fibre moyenne [206] ed i centri di curvatura dell'evoluta dell'ellisse $\left[\mathrm{I}_{62}\right]^{85}$ ) e della concoide di Nicomede ${ }^{86}$ ).

Sopra tutti questi emerge un congenere lavoro $[\mathrm{I} 60]^{8_{7}}$ ), nel quale il Mannheim, riprendendo da altro punto di vista il tema d'un suo lavoro giovanile [24], applicò i

83) Applications d'Analyse et de Géométrie, t. II (Paris, I864), p. II 7 .

84) Cfr. LoRIA, opera citata 57), p. 6 ; I.

${ }^{85}$ ) Cfr. anche [I52], pp. 20I-203 e [I84], p. 21.

86) L'Intermédiaire des Mathématiciens, t. II (1895), p. 224.

${ }^{87)}$ Cfr. [184], pp. 66-74. 
procedimenti a lui diletti allo studio delle caustiche piane, nell'ipotesi più generale in cui i raggi luminosi, invece di emanare da un punto, inviluppano una curva qualsivoglia. Anzitutto egli espose una notevole costruzione del punto di contatto d'un qualunque raggio rifratto col proprio inviluppo e ne desunse una relazione fra gli angoli d'incidenza e di rifrazione, l'indice di rifrazione, il raggio di curvatura in un punto della curva rifrangente e le lunghezze dei corrispondenti raggi incidente e rifratto; tale relazione porge la costruzione del centro di curvatura della caustica ed una formola che lega $\mathrm{i}$ raggi di curvatura in punti corrispondenti delle tre curve considerate (cioè curva rifrangente ed inviluppi dei raggi incidenti e rifratti). Non bastano questi pochi cenni a provare come anche la teoria delle caustiche sia debitrice all'infaticabile geometra di cui ci occupiamo di un notevole perfezionamento, anzi di un reale progresso?

Geometria dello spazio. - In un suo ben noto lavoro, STEINER ${ }^{88}$ ) ha ricorso ad un movimento infinitesimo per determinare il numero delle normali che possono condursi ad una curva piana algebrica da un punto qualunque del suo piano. Tale artificio logico fu esteso allo spazio da F. August ${ }^{89}$ ) ed il Mannheim se ne servi [49] per determinare la distribuzione nello spazio delle normali ad una superficie algebrica; ma, avendo egli ragionato esclusivamente sopra superficie generali ne' loro ordini e sopra curve intersezioni conplete di superficie, le proposizioni di geometria numerativa a cui pervenne hanno portata minore di quella posseduta dalle congeneri a cui giunsero G. Darboux ${ }^{90}$ ) e R. Sturm ${ }^{9 x}$ ). Nell'ipotesi che la superficie considerata sia di second'ordine [53] la stessa procedura conduce in modo uniforme ad un gruppo di teoremi sulle normali alle quadriche, alcuni dovuti a Desboves e Laguerre, ma in parte nuovi. Siaci lecito essere brevi su tali lavori che appajono scoloriti a paragone di altri che attualmente ci attraggono.

E noto che si chiama asse di curvatura d'una linea sgbemba l'intersezione di due suoi piani normali consecutivi; il Mannheim propose [58 ${ }^{9^{2}}$ ) di chiamare asse di curvatura d'una superficie sviluppabile l'intersezione del piano normale alla superficie condotto per una generatrice con l'analogo relativo alla generatrice consecutiva. Ricor-

88) Gesammelte Werke, Bd. II (Berlin I882) pp. $621-637$.

89) Geometrische Betrachtung der Normalen, welche sich von einem beliebigen Punkte auf eine algebraische Fläche fällen lassen [Journal für die reine und angewandte Mathematik, Bd. LXVIII (I868), pp. 242-245].

9o) Sur la surface des centres de courbure d'une surface algébrique [Comptes rendus hebdomadaires des séances de l'Académie des Sciences (Paris), t. LXX (I ${ }^{\text {er }}$ semestre I870), pp. I328-1333].

91) Ueber Fusspunkts-Curven und. Flächen, Normalen und Normalebenen [Mathematische Annalen, Bd. VI (1873), pp. 24I-263]; Ueber Normalen an algebraische Flächen [Ibid., Bd. VII (I874), pp. 567-582]; Zur Theorie der algebraischen Flächen [Ibid., Bd. IX (1876), pp. 573-575].

$9^{2}$ ) Cfr. la comunicazione preliminare [47]. Allo stesso periodo appartiene una breve Nota [7I] contenente la dimostrazione geometrica della proposizione seguente: $S e$ a partire da un punto a d'una superficie $(A)$, si tracciano su questa due curve aventi ivi un contatto d'ordine $n$, le normali di $(A)$ aventi per direttrici quelle curve banno fra loro un contalto dell'ordine $n+$ I nei centri principali di curvatura di $(A)$ situati sopra la normale in a a tale superficie. 
diamo pure che MONGE introdusse la considerazione dell'inviluppo dei piani normali d'una curva sghemba, a cui diede il nome di superficie polare della data curva o della corrispondente sviluppabile. Ora Mannherm ha osservato che la superficie polare dà luogo ad una nuova superficie analoga, da lui chiamata seconda superficie polare, e che, potendosi tale considerazione ripetersi indefinitamente, nasce tutta una serie di superficie polari successive ${ }^{93}$ ). Inoltre, egli propose di enunciare come segue il teorema di Meusnier: "quando due curve tracciate su una superficie hanno fra loro un contatto di primo ordine nel punto $a$, i loro assi di curvatura uscenti da tale punto passano per uno stesso punto "; la convenienza di questa nuova forma appare evidente considerando che guida alla seguente generalizzazione del teorema di MeusNier: quando due curve tracciate sopra una superficic banno fra loro nel punto a un contatto dell'ordine $n$, le loro $(n-\mathrm{I})^{m e}$ superficie polari banno ivi per assi di curvatura due rette passanti per uno stesso punto ${ }^{94}$ ).

Altri contributi dati da Mannherm alla teoria della curvatura delle superficie [59]: Sia $a$ un punto d'una superficie $(S) ; A$ la corrispondente normale; $b$ e $c$ i centri di curvatura di $(S)$ posti su $A ; B$ e $C$ le normali in $b, c$ alle due falde $(B),(C)$ dell'evoluta di $\left.(S)^{95}\right)$; su $B$ stanno due centri di curvatura $d$, $e$ di $(B)$ e su $C$ due $g, b$ di $(C)$; siano $D, E, G, H$ le corrispondenti normali della seconda evoluta di $(S)$; si chiamino $d^{\prime}, e^{\prime}$ i punti in cui $C$ è tagliata dai piani $(B, D),(B, E)$, e $A^{\prime}$ la tangente conjugata di $A$ relativamente alla superficie $(B)$; analoghi significati abbiano le lettere $A^{\prime \prime}, g^{\prime}, b^{\prime}$ : le rette $A^{\prime}, A^{\prime \prime}, B, C, d d^{\prime}, e e^{\prime}, g g^{\prime}, b b^{\prime}$ appartengono allo stesso paraboloide $\left.{ }^{96}\right)$. E questa una superficie importantissima, che s'incontra in molti lavori di Mannherm sotto il nome assai appropriato di paraboloide delle otto rette ${ }^{97}$ ). Fra le questioni di cui esso porge la soluzione citiamo a mo' d'esempio la seguente: dati gli assi di curvatura in un punto a di una superficie $(S)$ delle linee di curvatura passanti per a, nonchè i piani delle relative sezioni principali dell'evoluta di $(S)$, determinare $i$ centri di curvatura principali di questa superficie.

Le surriferite nozioni, combinate ai risultati di un precedente lavoro cinematico [48], posero in grado il Nostro di schizzare [6o] una teoria geometrica della curvatura delle superficie. Non pago di ció, subito dopo, dedicó un importante lavoro [6I] ai contatti delle superficie, teoria che, dopo Dupin, era rimasta stazionaria. Ora, se si prosegue nell'ordine delle idee in cui si aggirò questo celebre geometra, si è indotti a con-

93) Converrebbe determinare le equazioni di tali superficie, supponendo nota la rappresentazione analitica della sviluppabile considerata.

94) Cfr. [rs2], pp. 328-330.

95) Mannheim ha poi chiamate $B, C$ rette di curvatura relative al punto a di $(S)$, facendo notare come possano spesso vantaggiosamente surrogare l'indicatrice di DupiN.

${ }^{96}$ ) Se ne conosce l'equazione ? Venne già studiato il sistema degli $\infty^{2}$ analoghi paraboloidi relativi ai punti di una stessa superficie?

97) Veggasi ad es. la Memoria [99], ove, fra l'altro, si leggono altre due definizioni della stessa superficie. 
siderare, invece della solita conica indicatrice, una serie di curve di ordini costantemente crescenti; Mannheim propose di considerare invece le già definite rette di curvatura, il cui insieme costituiva ai suoi occhi l'elemento analogo nello spazio al centro di curvatura delle linee piane. Per porre in luce l'utilità di siffatta considerazione notiamo anzitutto che egli col mezzo di essa dimostró che la proposizione seguente, stabilita dal Dupis per $n=3$, sussiste eziandio per $n=4$ : Se le sezioni prodotte da $n$ piani arbitrari condotti per un punto a comune a due superficie, banno ivi un contatto d'ordine $n-\mathrm{I}$, lo stesso accadrà per qualunque altro piano condotto per a ${ }^{9^{8}}$ ). Aggiungiamo altri risultati che essa diede nelle mani del Mannheim: $\dot{E}$ una conica il luogo dei centri di curvatura in un punto a di una superficie $(S)$ delle evolute delle sezioni pro. dotte in questa da piani per una delle tangenti di $(S)$ in a. Due superficie $(S),\left(S^{\prime}\right)$, banno fra di loro un contatto di terzordine in un punto comune a se le falde delle loro evolute sono fra loro osculatrici ne' punti corrispondenti ad a, oppure se le loro linee di curvatura incrociate in a banno ivi un contatto di teríordine. Ecc.

La teoria della curvatura delle superficie essendo in ultima analisi lo studio del sistema costituito dalle sue $\infty^{2}$ normali, è naturale che il Mannheim sia stato condotto, dalle investigazioni di cui indicammo teste alcuni risultati, ad occuparsi dei sistemi di rette doppiamente infiniti, a cui il Kummer nel I859 aveva dato forma definitiva con una classica memoria, che una traduzione francese del Dewulf aveva resa conosciutissima in Francia ${ }^{\text {s9 }}$ ). Ed in alcune comunicazioni fatte alla Società filomatica nelle sedute del 2 e del 23 aprile e del 7 maggio 1864 indico a grandi linee un metodo per trattarla geometricamente ${ }^{\text {roo }}$ ). A base di esso stanno alcuni nuovi concetti che, convenientemente estesi, vennero tosto inseriti ed applicati da J. DE LA Gourniere nell'ull'ultimo volume del suo ben noto Traité de Géométrie déscriptive e che ritrovansi nel $§$ I d'una elaborata Memoria ([64]; cfr. [5o]) meritevole d'un cenno non fugace da parte nostra.

Sia $G$ una generatrice qualunque di una superficie rigata, o un punto fisso di $G$ e $\omega$ il relativo piano tangente; si chiami $y$ la distanza di $o$ da un punto qualunque $a$ di $G$, ed $Y$ l'angolo che il corrispondente piano tangente forma con $\omega$. In virtù di un noto teorema di Chasles sussisterà una relazione della forma $y \cdot \operatorname{tg} Y+\lambda . y+\mu . \operatorname{tg} Y=0$, ossia, se si pone $x=\frac{y}{\operatorname{tg} Y}, y+\lambda x+\mu=0$. Ora, se in questa relazione si considerano $x, y$ come coordinate cartesiane ortogonali di un punto di un piano $\alpha$ passante per $G$, essa rappresenta una retta a cui il Mannherm pose il nome di retta ausiliare; se si osserva che, condotta dal punto a nel piano $\alpha$ la retta perpendicolare a $G$ e dettane $a^{\prime}$ l'intersezione col piano ausiliare, si ha $Y=$ ang $a$ o $a^{\prime}$, si vedrà che la retta au-

$9^{8}$ ) Questa ipotetica proposizione venne già dimostrata in generale?

99) Nouvelles Annales de Mathématiques: Ière série, t. XIX (1860), pp. 362-37r; t. XX (I86r), pp. 72-76; 255-260, 359-365. IIème série, t. I (I862), pp. 3I-4I, 82-102.

roo) E noto che a Morius appartiene un metodo anteriore (I862) per conseguire il medesimo scopo; esso differisce da quello del MannHeim, a cui sembra essere rimasto ignoto. 
siliare porge un'immagine geometrica assai espressiva dell'interdipendenza fra i punti di $G$ ed i corrispondenti piani tangenti. Il Nostro se ne è giovato per stabilire le più essenziali proprietà delle rigate e risolvere i relativi problemi. Se $(\$$ II), invece di considerare le sole rette d'una rigata, si considera un sistema tale che "ciascuna (retta) sia determinata da un suo punto " ([64], p. I2I), ogni elemento $r$ del sistema ne ha $\infty^{\mathrm{I}}$ infinitamente vicine, le quali costituiscono un pennello di raggi, di cui il ManNherm insegna la rappresentazione ${ }^{\text {Ior }}$ ) sopra un cerchio passante pei fuochi di $r$ e di cui ritrova le proprietd fondamentali dovute a Malus, C. Sturm, Hamilton e Kummer, aggiungendovene di nuove ${ }^{\text {Ios}}$ ). Supponendo finalmente ( $($ III) che le rette considerate siano normali ad una stessa superficie si ottiene un noto ed importantissimo sistema di raggi, le cui rigate sono le normale della data superficie; applicandovi la precedente teoria generale appajono sotto nuova luce i teoremi sulla curvatura delle superficie scoperti da Monge, Dupin, Bertrand, Bonnet, Joachimsthal, Catalan, Lamarle, Gilbert, Picart, de La Gournerie e se ne ottengono altri; fra i nuovi risultati citiamo la dimostrazione diretta di una bella relazione che il Mannheim altrove [39] aveva dedotta dalla celebre formola di Eulero, le proprietà [II7] del pennello di raggi polare di un dato ${ }^{\text {ro3}}$ ) ed il seguente utile ed elegante teorema: Se dai punti centrali delle superficie elementari d'un pennello di raggi si conducono le normali a tali superficie, si ottengono le generatrici d'una conoide di PLücker.

I medesimi concetti condussero [65] il Nostro ad un'importante relazione scoperta da Bertrand fra le posizioni delle normali condotte ad una superficie dagli estremi di due archi infinitesimi eguali uscenti dal medesimo punto d'una superficie, e gli permisero ([66]; cfr. [62]) di trattare con metodo prettamente geometrico la celebre questione (che il SaInt-VEnant aveva proposto ed il Bertrand ha per primo risoluto) se due curve possano avere a comune il sistema delle normali principali. Così egli, non solo pervenne alla nota relazione caratteristica

$$
\frac{a}{\operatorname{tang} \alpha} \frac{\mathrm{I}}{r}+\frac{a}{\rho}=\mathrm{I}
$$

fra la flessione $\left(\frac{\mathrm{I}}{r}\right)$ e la torsione $\left(\frac{\mathrm{I}}{\rho}\right)$ di una curva di Bertrand ${ }^{\text {I04}}$ ), ma stabili

IoI) Cfr. [I 52$],$ p. 284.

ro2) Cfr. K. ZIEDLER, Liniengeometrie mit Anwendungen, II. Bd. (Leipzig I906), p. 80.

ro3) EsEmPIo. - Date due superficie $(A),(B)$, si conduce da un punto fisso o una trastersale a segarle in $a, b$ : sia $D$ l'intersezione dei corrispondenti piani tangenti. Se ora si fa ruotare quella trasversale attorno ad o, $D$ genera un pennello $i$ cui fuochi $f_{1}, f_{2}$ godono la proprietà che le rette a $f_{\mathrm{I}}$, a $f_{2}$ sono tangenti coniugate di $(A)$, mentre $b f_{1}, b f_{2}$ lo sono di $(B)$.

r04) Cfr. anche [105]. Gli è probabilmente nel corso di tali ricerche che il MannhEIM fu condotto a concepire la seguente questione, che crediamo tutt'ora insoluta: Come si puó generare una curva gobba tale che, in un suo punto qualunque, $i$ due raggi di curvatura abbiano fra loro una data relanione? [L'Intermédiaire des Mathématiciens, t. I (1894), p. 7]. 
le seguenti nuove relazioni

$$
r r_{\mathrm{t}}=\frac{a^{2}}{\operatorname{sen}^{2} \alpha}, \quad\left(\mathrm{I}-\frac{a}{\mathrm{f}}\right)\left(\mathrm{I}+\frac{a}{p_{\mathrm{I}}}\right)=\cos ^{2} \alpha
$$

che passano fra le prime e le seconde curvature in punti corrispondenti di due curve aventi le stesse normali principali; fra gli altri risultati conseguiti dal nostro geometra notiamo la costanza da lui avvertita del birapporto formato dai due punti in cui due curve di Bertrand sono incontrate da una comune normale principale e dai corrispondęnti centri di prima curvatura. Più tardi egli ritornó sopra tali curve per segnalarne nuove proprietà [ $\left.\mathrm{IO}_{4}\right]$ e per indicare [ $\left.\mathrm{I} 49\right]$ una costruzione elegante che serve a dedurre, l'uno dall'altro, i centri delle sfere osculatrici in due punti corrispondenti di due curve aventi le stesse normali principali. Il valore di tutti questi risultati sembraci tale da esigere che $\mathrm{i}$ futuri espositori della geometria differenziale ne tengano maggior conto di quanto abbiano fatto sino ad ora.

Dopo questa digressione, torniamo alla teoria della curvatura delle superficie, per segnalare la seguente notevolissima formola, scoperta dal Masnheim [79], che lega i raggi principali di curvatura $r_{1}, r_{2} ; t_{1}, t_{2}$ delle due falde $(B),(C)$ dell'evoluta d'una superficie $(S)$ nei punti $b, c$ in cui essa è toccata dalla normale $A$ di $(S)$ in un suo punto qualunque $a$, ai raggi principali di curvatura $R_{1}, R_{2}$ di $(S)$ in $a$ :

$$
4\left(R_{1}-R_{2}\right)^{2}+\left(r_{1}-r_{2}\right)\left(t_{1}-t_{2}\right) \operatorname{sen} 2 \beta \cdot \operatorname{sen} 2 \gamma=0
$$

ove $\beta, \gamma$ sono gli angoli che la normale $A$ forma con gli assi focali delle indicatrici in $b, c$ risp. di $(B),(C)$.

Degne di menzione ci sembrano anche le soluzioni date dal Mannheim $[77],\left[7^{8}\right]^{\text {ro5}}$ ) pei seguenti problemi :

I. Dati $i$ piani delle sezioni principali di una superficie $(S)$ in un suo punto a $e$, sulla corrispondente normale $A$, i relativi centri principali di curvatura, costruire il centro di curvatura in a dell'intersezione di $(S)$ con un piano condotto arbitrariamente per $a$.

II. Si projetti ortogonalmente una superficie $(S)$ su di un piano e si chiami a' la projezione di un punto a; costruire per la projezione del contorno apparente il raggio di curvatura $r$ in a' supponendo noti $i$ raggi principali di curvatura $R_{1}, R_{2} d i(S)$ in a.

Riguardo alla soluzione del $I^{0}$ va segnalata la sua indipendenza dai teoremi di Meusnier ed Eulero e riguardo a quella del $\mathrm{II}^{\circ}$ che essa guida spontaneamente alla bella relazione

$$
r=R_{1} \operatorname{sen}^{2} \omega+R_{2} \cos ^{2} \omega
$$

(w) essendo l'angolo che l'asse focale dell'indicatrice di $(S)$ nel punto considerato fa col piano di projezione.

Si connettono a questi altri lavori [82], [96 ${ }^{\text {ro6 }}$ ) in cui in due differenti modi è costruita la sfera osculatrice in un punto dell'intersezione di due superficie: una di tali costruzioni ha per fondamento la suesposta generalizzazione del teorema di MeusNiER,

I 5) Cfr. [I52], pp. 30r, 32I-322.

I06) Cfr. [I52], pp. 330-33 I. 
mentre l'altra esige sappiasi costruire il centro di curvatura dell'evoluta della sezione prodotta in una superficie da un piano qualunque ${ }^{107}$ ). Altrettanto nuove, forse meno importanti, ma per fermo più difficili, sono le congeneri questioni di cui leggonsi delle soluzioni eleganti in altri scritti del Mannheim, [84], [85], sui quali a malincuore sorvoliamo. Come corollario di una di tali soluziori egli ha ottenuto [86] per via geometrica questo bel teorema del RiBaucour: il raggio di curvatura geodetica in un punto di una linea di curvatura geodetica costante d'una superficie è eguale a $\frac{2}{3}$ del raggio di curvatura geodetica della sezione piana ivi tangente ed iperosculata da un cerchio ${ }^{\text {ros }}$ ). $\mathrm{E}$ va notato che tale teorema, essendo stato poi dedotto dal LAGUERre da un'antica formola da lui prima scoperta ${ }^{\text {I09}}$ ), al Mannherm punse vaghezza di mostrare [93] come a tale formola guidassero anche i propri metodi d'indagine.

Le rette normali ad una superficie essendo normali a tutto un sistema di superficie fra loro parallele, il Mannhen venne spontaneamente trascinato ad occuparsi della corrispondenza per parallelismo di due superficie qualunque [133]; e sopra un tema che poteva annoverarsi fra quelli esauriti scrisse una memoria che riscosse l'unanime plauso della Societa matematica di Londra, a cui venne presentata. Gli fu dato di cogliere tali nuovi allori, non solo servendosi delle risultanze di precedenti suoi scritti [68], [9I], ma anche introducendo le considerazioni di curve che fino allora non si erano trattate da tale punto di vista ${ }^{\text {Io }}$ ). Merita di venire anche rilevata un'espressione data ivi dal MANNHEIM pel raggio di curvatura dell'evoluta del contorno apparente di una superficie qualunque, della quale più tardi egli diede una dimostrazione, fondata sull'uso delle due rette di curvatura, in una nota $[\mathrm{I} 65]^{\mathrm{III}}$ ) contenente complementi preziosi alla memoria sulle superficie parallele.

L'esteso lavoro [62] di cui più sopra rendemmo conto non è l'unico che il Nostro abbia consacrato alla Geometria infinitesimale dello spazio rigato; un altro egli ne scrisse [143], che sottopose al giudizio dell'Accademi: dei Lincei. Il parere su di esso pronunciato dai Commissari all'uopo delegati, Beltrami e Cremona, è compendiato in una Re-

107) Riguardo a questo nuovo problema si vegga, oltre ad una comunicazione [83] fatta dal Mannheim alla Società filomatica di Parigi addi 24 giugno 1874 , il lavoro [165].

$\left.{ }^{108}\right)$ Notiamo qui che alla curvatura geodetica si riferisce un altro breve lavoro [122], che ci limitiamo a citare, non contenendo novità sostanziali.

I09) LAGUERRE, Sur une propriété relative aux courbes tracées sur une surface quelconque [Bulletin de la Société Philomathique de Paris, t. VII (1870), pp. 49-51]; Sur un théorème de Géométrie [Comptes rendus hebdomadaires des séances de l'Académie des Sciences (Paris), t. LXXX ( ${ }^{\text {er }}$ semestre 1875), pp. 822-823]. Ovvero: CEuvres de Laguerre, t. II (Géomítrie) (Paris, I905), pp. I29-I 30, $420-421$.

I Io) Tali sono le curve già investigate dal DE LA Gournerie (Traité de Géométrie descriptive, $3^{\mathrm{e}}$ Partie, p. 96) e dal Ribaucour \{Proprittés de courbes tracées sur les surfaces [Comptes rendus hebdomadaires des séances de l'Acadímie des Sciences, t. LXXX ( ${ }^{\text {er }}$ semestre I875), pp. 642-645]\}, che ammettono in ogni punto un cerchio iperosculatore e quelle per le quali è costante la curvatura normale.

III) Cfr. [184], pp. 550-577. 
lazione ${ }^{{ }^{r 2}}$ ) presentata dal primo a nome di entrambi nella seduta del I2 Aprile I885, e che qui riproduciamo integralmente a titolo di onore pel geometra di cui ci occupiamo, convinti che ai nostri lettori tornera gradito il trovar qui uno squarcio della prosa armoniosa e robusta d'un estinto illustre e caro:

"Il problema geometrico della determinazione degli elementi delle superficie caustiche è risoluto completamente, e nel caso piu generale, per la prima volta in questa Memoria, sebbene sia stato oggetto di anteriori ricerche da parte di altri geometri.

"Il problema delle caustiche fu posto per la prima volta da TschiRnhausen, il quale considero una serie di raggi luminosi paralleli incidenti su di una curva piana e ne ricercó l'inviluppo dopo la riflessione. Giovanni Bernoulli studiò le caustiche per rifrazione: DE la Hire, Leibnitz, de L'Hópitai, CoRnu continuarono queste ricerche. Il problema fu trasportato allo spazio da Malus, il quale considerò un sistema di raggi uscenti dai punti di una superficie qualunque secondo una legge analitica qualsivoglia; e dimostrò che questi raggi toccano due superficie che chiamó caustiche. Per tal modo la questione entro in quella fase importantissima che ha inaugurato la teoria dei sistemi di rette. Hamitron, nella sua Theory of systems of ray's, chiamò pennello di raggi il fascio costituito da una retta qualunque del sistema e da tutte quelle ad essa infinitamente vicine: e ne studiò le proprietà. Kummer, proseguendo in questo indirizzo, completo le ricerche di HAmiton e svelò l'intimo legame esistente fra lo studio dei sistemi di rette e la teoria della curvatura delle superficie.

"Il sig. Mannheim, cosi noto nel mondo scientifico per le sue ricerche di geometria infinitesimale e di geometria cinematica, ha dato per il primo una teoria geometrica dei pennelli di raggi, in una Memoria presentata all'Académie des Sciences nel 1870: Mémoire sur les pinceaux de droites et les normalies, contenant une nouvelle exposition de la thiorie de la courbure des surfaces ${ }^{113}$ ); la qual Memoria è cosi intimamente legata con quella di cui stiamo per rendere conto, che non possiamo esimerci dal darne qualche cenno. L'autore comincia col riprendere la formola di Chasles che fornisce la legge di variazione dei piani tangenti ad una superficie rigata nei punti d'una sua generatrice, e traduce questa formola in una rappresentazione geometrica della legge stessa, mediante una retta che egli chiama retta ausiliare relativa ad un punto della generatrice data. Cio posto, egli considera in un sistema di raggi tutti gli elementi di superficie rigata compresi fra un raggio qualunque e ognuno di queili infinitamente vicini (superficie elementari del pennello); ed applicando a queste superficie elementari la rappresentazione mediante la retta ausiliare ottiene, con metodo geometrico uniforme, tutti i teoremi di Malus, Hamilton, Kummer, Sturm, etc., oltre a nuove proprietà. Passa quindi a supporre che il pennello di raggi sia formato di normali ad una superficie: è da questo caso che la teoria della curvatura discende naturalmente. Tutti i classici teoremi di Monge, Euler, Meunier, DupIN, ecc., sono cosi riprodotti mediante elegantissime dimostrazioni e indipendentemente da ogni processo analitico.

( Frattanto era stato posto il problema della determinazione degli elementi di curvatura delle superficie caustiche per rifrazione, e importanti contribuzioni analitiche alla sua soluzione erano state date da Bertrand, e in particolare da Sturm, prima nella sua Memoria sull'Ottica e poi nell'altra interessantissima sulla Visione. E di questo problema che il sig. ManNHEIM offre pel primo una soluzione geometrica completa nella Memoria presentata alla nostra Accademia. Le notizie precedenti ce ne agevoleranno la relazione.

ina) Beltrami e Cremona, Relazione sulla Memoria del sig. A. Mannheim: "Mémoire d'optique géométrique, contenant la théorie du point représentatif d'un èlément de surface réglée et son emploi, tant pour la démonstration nouvelle de théorèmes relatifs à la courbure des surfaces, que pour la determination plane des éléments des surfaces caustiques" [Atti della R. Accademia dei Lincei, serie IV, Memorie della Classe di Scienze Fisiche, Matematiche e Naturali, vol. I (1885), pp. 517-519].

${ }^{13}$ ) Journal de Mathématiques pures et appliquées, II ${ }^{e}$ série, tome XVII (I872), pp. Iog-I66. 
“ L'autore comincia col migliorare la rappresentazione geometrica della legge di variazione dei piani tangenti ad una superficie rigata nei punti di una generatrice. La retta ausiliare, di cui egli faceva uso nella prima Memoria, dipendeva dalla scelta di un punto della generatrice: dimodochè per una data generatrice si avevano infinite rette ausiliari, le quali peró passavano tutte per uno stesso punto. Ora l'autore, abbandonando le rette ausiliari, adotta questo punto che egli chiama punto rappresentativo. E noto che, data una generatrice $r$ d'una superficie gobba, la distanza di un suo punto qualunque dal punto centrale è in rapporto costante colla tangente trigonometrica dell'angolo che il piano tangente in quel punto fa col piano centrale : ciò posto, il punto rappresentativo è situato sopra una perpendicolare ad $r$ elevata dal punto centrale ed è distante da questo di una quantita eguale a quel rapporto costante. Il punto rappresentativo permette di costruire immediatamente per ogni punto di $r$ il suo piano tangente.

"Siccome la sostituzione del punto rappresentativo alle rette ausiliari abbrevia di molto le considerazioni, il sig. Mannhem riproduce la sua teoria geometrica della curvatura delle superficie introducendori notevoli semplificazioni: e questa è una parte molto interessante della Memoria. La relazione di Euter, le proprietà dell'indicatrice di Dupin, infine tutti i teoremi più importanti della teoria, sono dedotti con grande eleganza.

« L'autore riprende poi lo studio dei pennelli di raggi. Si consideri il pennello formato da tutti i raggi di un sistema vicinissimi ad un raggio $r$. Ogni superficie elementare del pennello possiede il suo punto rappresentativo sopra un piano qualunque condotto per $r$; il luogo di questi punti è una circonferenza, la quale, nel caso che il pennello sia formato di normali, ha per diametro il segmento compreso tra i fuochi di $r$. Sulla circonferenza esiste un certo punto la cui posizione dipende dall'orientamento del pennello rispetto al piano scelto. Quando sono dati quella circonferenza e quel suo punto, il pennello di raggi è perfettamente conosciuto: se ne ha dunque cosi, in certo modo, una rappresentazione piana. Questa maniera di rappresentazione era gia stata accennata dal sig. MANNHEIM in una sua comunicazione all'Académie des Sciences del 9 giugno 1879: ma qui é svolta più completamente.

" Tutto il materiale accumulato in questa prima parte della Memoria, giova all'autore per la risoluzione del problema di ottica geometrica di cui abbiamo fatto menzione e che puó essere enunciato cosi : dati gli elementi di un pennello di raggi incidenti e gli elementi di curvatura della superficie separante i due mezzi, determinare gli elementi del pennello dei raggi rifratti. 11 metodo consiste nel sostituire ai due pennelli le loro rappresentazioni piane e cercare le relazioni che le legano. $\mathrm{Ci}$ duole di non potere in questa Relazione seguire l'autore in tutta la serie di considerazioni che gli permettono di dare, oltre ad una soluzione, per dir cosi fondamentale, due o tre varianti di eguale importanza. Cio che importa di notare è che questi procedimenti si risolvono in parecchie costruzioni pane degli elementi delle superficie caustiche, e che perciò essi dànno alla soluzione del problema quella massima esplicazione che non puó venirgli che dalla geometria. Del resto l'autore ha potuto anche dedurre dalle costruzioni geometriche il calcolo degli elemcnti del pennello rifratto, in modo senza dubbio assai più semplice di quello che darebbero gli ordinari metodi di Geometria analitica infinitesimale. E con questo calcolo e con alcune note esplicative, che termina il lavoro.

" La Memoria è assai interessante, non solamente per l'importanza dei risultati contenuti nella seconda parte, ma anche per l'eleganza di quei metodi geometrici che il chiarissimo autore adopera anche questa volta con mirabile felicità e che meritano di essere meglio conosciuti e coltivati in Italia. Per queste ragioni siamo lieti di proporre la integrale pubblicazione della Memoria del sig. ManNHEIM nei nostri Atti, e di dare cosi un meritato tributo di stima al valoroso geometra francese, che ha tanto contribuito a mantenere in onore la pura Geometria ».

Come è opportunamente rilevato dal Beltrami i germi dei concetti fondamentali applicati dal Mannheim alla teoria delle caustiche nello spazio si trovano in altre più antiche sue memorie relative alle superficie rigate: sono quelle in cui, dopo di avere [IO5] sugg erito di rappresentare una superficie rigata mediante l'inviluppo delle sue rette ausi- 
liari delle singole sue generatrici, viene mostrato [106], [107] in qual modo si possano ottenere le superficie rigate di nota rappresentazione; rileviamo anche [ I I 2] l'osservazione che, per studiare una curva sghemba, giova considerarla come situata sopra la rigata delle normali principali, da rappresentarsi poi col metodo testè esposto ${ }^{\text {II4}}$ ).

Nella contemporaneità di tali studi con altri sulla superficie delle onde, dei quali tratteremo fra breve, è da ricercarsi la prima radice di un nuovo modo di trasformazione delle superficie rigate immaginato dal Mannheim [I24]: per applicarlo si suppongano dati un punto fisso o ed una rigata $(G)$ e si consideri il piano determinato da o e da una generatrice $G$ di tale superficie; facendolo ruotare d'un angolo retto attorno ał $o$, $G$ assumerd una posizione nuova $G_{\mathrm{I}}$ e la data rigata si trasformerà in altra $\left(G_{1}\right)$. Il lettore avvertirà tosto l'applicabilità di questo metodo a tutte le rette dello spazio; facendolo agire sopra un pennello di raggi, nasce un nuovo pennello legato al primo da notevoli relazioni [ 125$]$.

La bella collezione di lavori del Mannherm sulle superficie rigate contiene ancora altri scritti ${ }^{\text {II5 }}$ ). Uno di essi [I03] ha per iscopo la ricerca di una relazione fra il parametro di distribuzione $k$ relativo ad una generatrice $G$ di una rigata $(G)$ ed il parametro di distribuzione $k_{\mathrm{I}}$ relativo ad una generatrice $N$ del paraboloide formato dalle normali di $(G)$ lungo $G$; ora Mannherm, ricorrendo ad una opportuna rappresentazione $\mathrm{di}(G)$ in doppia projezione ortogonale, trova $k_{\mathrm{I}}=\frac{k}{\cos ^{2} \theta}, \theta$ essendo l'angolo che $N$ forma con la normale alla superficie $(G)$ nel punto centrale della generatrice considerata. Fra i corollari di tale notevole relazione citiamo il seguente: il raggio di torsione in un punto d'una curva gobba è eguale a ciascuno dei raggi principali di curvatura in quel punto della rigata costituita dalle normali principali della curva. $\mathrm{Da}$ un altro lavoro [II3], próvocato da un articolo di J. FRANke Sur la courbure des surfaces réciproques ${ }^{\mathrm{II}}$ ), si apprende un'elegante relazione fra i parametri di distribuzione di due rigate dedotte una dall'altra con uno speciale procedimento. Un terzo [ I I 5 ], riferentesi ad un passo del grande Mémoire sur la théorie générale des surfaces di $\mathrm{O}$. BONNET ${ }^{\text {II7}}$ ), ha per iscopo di stabilire geometricamente che se una linea tracciata sopra una superficie rigata gode di due delle seguenti prerogative, possederà anche la terza: è una geodetica, è la linea di stringimento, è una trajettoria obliqua delle generatrici. Finalmente, quello di data più recente $[\mathrm{I} 75]$ fa acquistare un'insperata generalita ad un teorema avvertito da $\mathrm{C}$. Broche ${ }^{\text {II }}$ ) nelle rigate generate da una retta invariabilmente connessa al triedro principale di una linea gobba.

II4) Le quatre Note or citate trovansi riprodotte in [184], pp. 532-54I.

xi5) Alle rigate si riferisce anche la Questione 823 delle Nouvelles Annales de Mathématiques [IIeme série, t. VI (I867), p. 336].

156) Journal de Mathématiques pures et appliquées, IIIème série, t. III (I877), pp. 4I5-42I.

${ }^{117}$ ) Journal de l'École Polytechnique, Iere série, XXXII Cahier (I848), pp. I-I46.

${ }^{118}$ ) Sur les surfaces réglées qui passent par une courbe donnée [Comptes rendus hebdomadaires des séances de l'Académie des Sciences, t. CX (i890), pp. 5I5-5I6]. 
Le rigate non sono le uniche superficie speciali che abbiano attratta l'attenzione del Mannheim. Infatti, nell'elenco dei suoi lavori troviamo una Nota [63] sulla superficie luogo dei punti equidistanti di due rette fisse, della quale eransi già occupati J. A. Serret ed E. Catalan; riguardo ad essa egli ha insegnate delle costruzioni semplici e notevoli, non foss'altro perchè sussistono anche supponendo quelle distanze non eguali, ma in rapporto costante. Al Mansheim spetta poi il merito ([Ioo], [IO2]) di avere per primo investigate geometricamente le superficie i cui raggi principali di curvatura in un punto qualunque sono legati da una relazione e di avere aggiunta qualche nuova proprieti a quelle notate dal Ribaucour e dall'Halphen. Inoltre: esistono in un ellissoide $\infty^{2}$ corde $a b$ di data lunghezza e tali che le normali alla superficie nei loro estremi si tagliano, i loro centri costituiscono una superficie considerata da J. W. L.

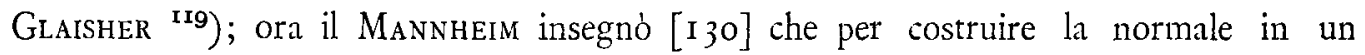
suo punto qualunque $m$ basta unire questo all'intersezione delle normali in $a, b$ all'ellissoide, costruzione non soltanto elegantissima, ma degna di nota per la sua identita con quella che dà la normale al luogo dei punti medi delle corde di data lunghezza d'un'ellisse. Finalmente egli ha insegnato [II9] ${ }^{\text {I20 }}$ ) a costruire semplicemente $i$ centri di curvatura principali in un punto qualsivoglia d'un'elicoide rigata, apportando cosi un rilevante perfezionamento alla teoria di una superficie, per importanza teorica e pratica a nessuna seconda.

\section{Superficie delle onde ${ }^{\mathrm{xa}}$ ).}

Ma la superficie a cui egli si dedicó col massimo impegno per circa un trentennio, applicandovi tutti i metodi di cui disponeva, cinematici e geometrici, infinitesimali e projettivi, è la supetficie delle onde; quali e quanti risultati egli abbia ottenuti emerge dal seguente compendio dei relativi lavori ${ }^{122}$ ).

I primi geometri che, a partire dal FreSNel (I822, I828, I83I), si occuparono di tale superficie la investigarono in base alla sua rappresentazione analitica; ma, sino dal I833, il MAC-CullaGH ${ }^{223}$ ) mostró come fosse possibile e relativamente agevole lo stabilirne le proprietà col solo sussidio della Geometria. Gli è appunto nella via da lui aperta che si pose il ManNheim, il quale, nelle sue prime pubblicazioni sull'argomento

II9) On a space-locus connected with the ellipsoid [Quarterly Journal of Mathematics, t. XV (I879), pp. 283-294].

120) Cfr. [152], p. 396.

I21) Per particolari storici sull'argomento si veda [152], p. 267; inoltre: Chasles, Rapport sur les progrès de la Giométrie (Paris, 1870 ), pp. 47-54; E. WoLfFING, Bericht über den gegenwärtigen Stand der Lehre von der Fresnel'schen Wellenfläche [Bibliotheca Mathematica, serie III, t. III (I902), pp. 36I-382]; G. LoRIA, Il passato ed il presente delle principali teorie geometriche, $3^{\mathrm{a}}$ edizione (Torino, 1907).

${ }^{\text {I22) }}$ Per una parte di questi, veggasi C. Niven, On Mr. Mannheim researches on the wave Surface [Quarterly Journal of Mathematics, t. XV (1878), pp. 242-257].

I23) The collected Works (Dublin, I880), pp. 2I e seg. 
$\left.[44],[45]^{\mathbf{r 2 4}}\right)$, prese le mosse dalla seguente definizione della superficie: “Sia $m$ un punto d'un ellissoide $(m)$ di centro o e $m n$ la corrispondente normale alla superficie; si conduca per $o$ nel piano o $m n$ il segmento $0 m_{1}$ perpendicolare alla retta $o m$ ed eguale a tale segmento; il luogo geometrico del punto $m_{\mathrm{r}}$ è la superficie delle onde $\left(m_{1}\right)$ ); la normale ad essa in $m_{1}$ si ottiene, come notarono Mac-Cullagh e Plücker, conducendo da tale punto la perpendicolare ad $m n$. Se $m$ si muove descrivendo sul dato ellissoide una linea qualunque, $m_{1}$ descriverà una curva sulla superficie d'onda e il Mannheim ha insegnato a dedurre dalla tangente alla prima la tangente alla seconda; di più ha indicate delle costruzioni semplici pei centri di curvatura e per le direzioni delle linee di curvatura in un punto arbitrario di $\left(m_{1}\right)$, desumendone qualche nuova proprietà di questa superficie. Tali costruzioni, benchè non complicate, sono suscettibili di ulteriori semplificazioni, le quali si apprendono dall'elaboratissima pubblicazione che il Nostro dedicó alla memoria di D. Chelini [I3I].

In lavori posteriori ([76]; cfr. [80]) il MannheIm preferi considerare la superficie d'onda come inviluppo dei suoi piani tangenti, ciascuno dei quali è parallelo ad un piano diametrale dell'ellissoide dato e ne dista di una lunghezza inversamente proporzionale ad un semiasse della corrispondente sezione ellittica; egli giunse cosi a parecchie nuove proposizioni, fra cui spiccano le seguenti:

I. Si conducano ad un ellissoide ed alla corrispondente superficie delle onde dei piani tangenti fra loro paralleli; $i$ diametri di tali superficie che vanno ai relativi punti di contatto incontrano uno dei detti piani in quattro punti che, essendo vertici d'un rettangolo, appartengono ad un cercbio; il diametro perpendicolare ai piani suddetti e quello passante pel punto di contatto del piano tangente all'ellissoide passano per gli estremi d'un diametro di quel cerchio. I quattro diametri, di cui sopra, banno per tracce quattro punti d'un cerchio anche sopra un piano perpendicolare al diametro che va al punto di contatto dell'ellissoide con quel piano tangente.

II. Si conducano un diametro $D$, nonchè i piani tangenti negli estremi di esso all'ellissoide e alla superficie d'onda; $i$ diametri perpendicolari a questi piani tangenti segano quei piani tangenti nei vertici d'un rettangolo, epperó in quattro punti d'una circonferenza. Le tracce delle stesse rette sopra quel piano tangente all'ellissoide stanno eziandio su di un cerchio di cui un diametro ha per estremi il relativo punto di contatto ed il piede del diametro perpendicolare a quel piano.

La surricordata costruzione delle normali alla superficie d'onda abilita anche a determinare [88] le normalie dell'ellissoide a cui corrispondono sulla superficie delle onde delle normalie sviluppabili, questione che puó anche trattarsi, vuoi [I I6] ${ }^{\mathbf{1 2 5}}$ ) col sussidio della retta ausiliare dal Mannheim introdotta per dare un'immagine sensibile della variazione del piano tangente ad una rigata nei punti d'una generatrice, vuoi [ I 26] col mezzo di un metodo, da noi gia segnalato, per trasformare le rigate, da lui altrove [I 24], [1 25] applicato ai sistemi di $\infty^{1} \mathrm{o} \infty^{2}$ rette. Fra $\mathrm{i}$ risultati ottenuti basti riferire il seguente:

124) Cfr. [I52], pp. 232-234.

125) Cfr. [184], pp. $545-550$. 
La trasformata di una delle dette normalie mediante polarità rispetto ad una sfera concentrica al dato ellissoide è una rigata, la cui linea di stringimento è il luogo dei piedi delle perpendicolari calate dal centro di questo sulle generatrici.

Invece la costruzione per punti delle stesse superficie (v. sopra) suggerisce quest'altra questione: che cosa corrisponde, sopra la superficie delle onde, ad una linea di curvatura dell'ellissoide? Il Mannheim ha trovato [109] che le normali alla superficie d'onda in punti d'una delle linee richieste sono perpendicolari a diametri fra loro eguali del corrispondente ellissoide.

Gli elementi della superficie d'onda suscettibili d'immediata interpretazione ottica sono le distanze dal centro dei punti e dei piani tangenti della superficie; giacchè le une corrispondono alle velocità secondo i raggi efficaci, mentre le altre corrispondono alle velocità di propagazione normale delle onde piane. Era quindi della massima importanza il cercare delle relazioni fra tali elementi; gli è quello che fece il Nostro, in un gruppo di lavori ([87], [89], [92], [94]) ove trovansi applicate e combinate accortamente le surriferite generazioni della superficie come loogo e come inviluppo: ci duole di non potere, per l'estensione già assunta dal presente scritto, che riferire alcune delle interessantissime proposizioni da lui in tal modo scoperte: La somma dei quadrati delle inverse delle velocità di propagazione normale di una vibrazione qualunque e delle due onde corrispondenti alle vibrarioni parallele al piano di polarizzazione del raggio lungo cui si propaga la prima è costante; la velocità del raggio che propaga tale vibrazione varia in ragione inversa del prodotto delle velocità delle due onde; la somma dei quadrati delle velocità dei raggi efficaci relativi a queste onde è costante.

Fondandosi sul fatto ben noto che la superficie d'onda è tagliata secondo una conica ed un cerchio, tanto da ogni piano principale dell'ellissoide, quanto dal piano all'infinito, il Mannherm stabili [ro8], [ I I I] l'esistenza di dodici piani (tangenti singolari), ognuno dei quali tocca la superficie secondo un cerchio; egli aggiunse che la superficie è tagliata secondo una quartica anallagmatica da ogni piano parallelo ad un piano tangente singolare e che [15I] essa contiene anche altre analoghe curve sghembe, intersezioni delle superficie con certi coni quadrici ad essa concentrici.

Cercando un teorema analogo a quello secondo cui le normali d'un ellissoide sono segate in parti proporzionali dai piani principali della superficie, il Nostro trovó che $i$ punti in cui una normale alla superficie d'onda ne incontra $i$ piani principali formano un birapporto costante con la projezione su di essa del centro delle superficie ${ }^{\text {rô }}$ ). Egli si è anche occupato degli ombelichi della superficie in questione, e, senza conoscere un analogo lavoro precedente ${ }^{\text {I27 }}$ ), ne indicó una costruzione $[\text { I 23 }]^{\text {I28 }}$ ), dalla quale emerge che esistono otto tali punti, tutti reali ${ }^{129}$ ).

г26) Per altri teoremi sulle normali veggasi [157].

127) C. Galopin Schaub, Étude sur la théorie de la double réfraction [Annales des Sciences Physiques et Naturelles, II ${ }^{\mathrm{e}}$ série, t. XVIII (I863), pp. I3I-I44].

${ }^{128}$ ) Cfr. [I52], pp. 334-337.

129) S. RoBeRTs ha confermato analiticamente questo risultato nella Nota On some Forms of the Equation of the wave Surface [Quarterly Journal of Mathematics, Vol. XVII (I88I), pp. 319-327]. 
Alcune delle ricerche di Geometria cinematica di cui rendemmo gia conto lo guidarono poi a concepire un nuovo punto di vista da cui puó considerarsi la superficie d'onda [I28]. Egli osservò che, quando un solido non è soggetto che a cinque o quattro condizioni, ogni suo punto descrive una trajettoria lineare o superficiale, ma se invece le condizioni sono soltanto tre, ogni punto del solido è, in generale, suscettibile di $\infty^{3}$ posizioni; tuttavia esiste una superficie luogo dei punti ad ognuno dei quali non compete che una superficie trajettoria; altre figure analoghe derivano dal considerare le rette ed i piani dello spazio. Esempio: le $\infty^{3}$ corde d'un ellissoide che sono viste sotto angolo retto dal centro della superficie, nonchè gli $\infty^{3}$ centri di esse, ammettono come superficie limite una superficie d'onda. Da ció la seguente nuova generazione: se un angolo retto $a c b$ circoscritto ad un ellissoide $\dot{e}$ tale che il suo piano sia normale a questa superficie nei punti di contatto $a, b$ dei suoi lati, il suo vertice $c$ appartiene ad una superficie $d$ 'onda, di cui la normale in $c$ è la retta che lo congiunge al centro del segmento $a b$. E importante osservare che questa proposizione sussiste [129] supponendo che i lati dell'angolo circoscritto siano tangenti, non allo stesso ellissoide, ma a due ellissoidi omofocali. Essa puó evidentemente anche enunciarsi come segue: la superficie delle onde è il luogo dei centri dei coni quadrici circoscritti ad un ellissoide $(E)$ e di cui una sezione principale $\dot{e} \alpha=\frac{\pi}{2}$. Tale nuovo enunciato condusse il Mannheim a considerare [1 32] le superficie analoghe per cui $\alpha$ è ancora costante ma $\neq \frac{\pi}{2}$ e ad osservare che la superficie d'onda dedotta come si disse dall'ellissoide $(E)$ è tagliata da un ellissoide omofocale a questo secondo una sua linea di curvatura, mentre un iperboloide omofocale ad $(E)$ la sega, oltrechè in una linea di curvatura, in una linea sferica.

L'ultimo lavoro che il Mannherm dedicó alla superficie di cui ci occupiamo [194] ha per intento principale la dimostrazione di un teorema del Niven e la determinazione degli assi di una superficie d'onda di cui si conoscono i piani principali, un punto ed il relativo piano tangente ${ }^{\mathbf{1 3 0}}$ ). Non essendoci concesso di farne più di una fugace menzione chiuderemo questa parte della nostra analisi osservando come dagli studì di cui è parola il Mannheim sia stato indotto ad occuparsi ([195] v. anche [207]) delle superficie apsidali ${ }^{\mathbf{1 3 x}}$ ) e ad osservare che, per l'influenza di elementi eccezionali, non sempre, come ritenevasi, "se una superficie è apsidale d'un'altra, questa lo è della prima »; a lui risale dunque il merito di avere iniziato lo studio degli elementi singolari della trasformazione apsidale.

130) Un complemento alla Nota [I94] trovasi nella risposta data dal Nostro alla Questione I 267 del L'Intermidiaire des Mathématiciens [t. V (1898), pp. 238-240]. Aggiungasi che sotto il pseudonimo "CANON " egli ha risolta la Questione 1855 (da lui proposta) delle Nouvelles Annales de Mathematiques [III ${ }^{\mathrm{e}}$ série, t. XIX (I900), p. 382 ; IV érie, t. III (I903), p. 47I], di cui ecco l'enunciato: Un cono ba per vertice un punto d'un ellissoide e per base la sezione centrale il cui piano è normale al diametro dell'ellissoide passante per quel punto; l'inviluppo degli $\propto^{2}$ coni analoghi è una superficie delle onde.

${ }^{\text {r }}{ }^{\mathrm{I}}$ ) Cfr. Mac-Cullagh, The collected Works (Dublin, I880), p. $3 \mathrm{I}$.

Rexd. Circ. Matem. Palermo, t. XXVI ( ${ }^{\circ}$ semestre 1908). - Stampato il 22 febbrajo 1908. 


\section{Geometria projettiva.}

Il Mannheim, educato in un ambiente tutto impregnato dalle idee di Poncelet e di Chasles, non poteva escludere dalle proprie elucubrazioni le teorie che rampollano dal Traité des propriétés projectives des figures e dal Traité de géométrie supérieure. Prima di addentrarci nella disamina dei frutti di questo genere di studi, giova che rileviamo due cose; una è che alla Geometria superiore, moderna o projettiva che dir si voglia, appartengono in maggioranza le numerose questioni da lui proposte come esercizi nelle Nouvelles Annales de Mathématiques; l'altra che sin dal 1865 egli ha [38] fatta ed applicata l'osservazione che "non si altera il risultato della risoluzione d'un problema, facendo variare gli elementi che non sono interessanti nello sviluppo di tale soluzione », osservazione in cui puo forse ravvisarsi una forma embrionale del principio della conservazione del numero.

Coniche. - Una delle più belle ed importanti questioni offerte dalla teoria delle curve di second'ordine è la costruzione, in direzione ed in lunghezza, degli assi d'un'ellisse determinata da una coppia di diametri conjugati; si puó dire che ad essa il Mannheim debba se il suo nome cominciò ad essere conosciuto dagli studiosi. Infatti nel tomo IX (I850) delle Nouvelles Annales de Mathématiques leggesi (p. 419): "Il sig. Mannherm, allievo della Scuola politecnica, fa questa bella osservazione sopra un teorema noto: Quando due vertici $A, B$ d'un triangolo $A B C$ di grandezza costante si muovono sopra due rette fisse $O x, O y$, il terzo vertice $C$ descriverd un'ellisse col centro in $O$. Se si circoscrive un cerchio al triangolo $O A B$ e se ne unisce il centro ad $O$, dette $E, F$ le intersezioni della periferia di quel cerchio, le rette $O E, O F$ saranno le direzioni degli assi di quell'ellisse, mentre i segmenti $O E, O F$ saranno le lunghezze dei suoi semiassi ”. $\mathrm{Si}$ deve ritenere che il Nostro abbia continuato poi ad occuparsi di quella questione, giacchè, riordinando i materiali con cui compone il suo opus magnum [I84], egli trovó fra le sue vecchie carte una costruzione semplicissima per gli assi d'un'ellisse determinata da una coppia di diametri conjugati $[\mathrm{I} 2 \mathrm{I}]{ }^{\mathbf{r} 3 \mathbf{a}}$ ), da lui dedotta da una generazione cinematica dell'ellisse differente da quella surriferita, cioè considerando tale curva come trajettoria di un punto invariabilmente connesso ad un cerchio che muovesi toccando internamente un cerchio fisso di raggio doppio. Di. questa nuova costruzione egli mise in luce la relazione con una scoperta da ChasLes ${ }^{\mathbf{1 3 3}}$ ) e fece vedere, da un lato come essa conduca spontaneamente ai teoremi di Apollonio e dall'altro come ad essa si possa pervenire prendendo le mosse dalle più elementari proprietà delle coniche. Altre nuove costruzioni congeneri vennero dal Nostro dedotte [139] dalla seguente genesi delle coniche: " $\mathrm{E}$ dato un cerchio riferito ad una coppia di diametri fra loro ortogonali; sopra un'ordinata qualunque si costruisce un triangolo simile ad un dato: il luogo del terzo

${ }^{13^{2}}$ ) Cfr. [150], pp. 9-11. La Solution de la Question 366 (Nouvelles Annales de Mathématiques, t. XVI, p. I87-189) induce a fare risalire tale costruzione all'anno 1857.

r33) Aperçu bistorique, $2^{\mathrm{e}}$ édition (Paris, 1875), p. 362. 
vertice è un'ellisse ». Non meno semplici ed eleganti sono le soluzioni del medesimo problema che si leggono in un articolo semianonimo $\left[\mathrm{I}_{67}\right]^{\mathbf{1 3 4}}$ ) e che poggiano sopra considerazioni cinematiche diverse dalle precedenti.

Con ció non abbiamo esaurito l'elenco dei lavori attestanti l'interesse del Nostro per le curve di second'ordine. Infatti a lui devesi un'elegante dimostrazione geome-

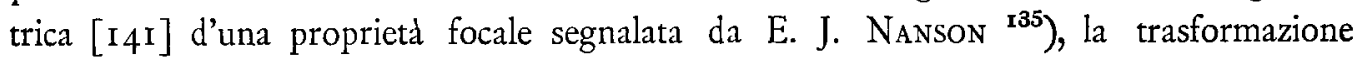
di essa per polarita ed una dimostrazione analoga [I92] per un teorema di CAYLEY relativo al punto in cui un cerchio di curvatura dell'ellisse taglia nuovamente la curva.

Maggiore originalità ed importanza possiedono le richerche da lui fatte sulla curvatura delle coniche; alcune $[\mathrm{I} 74]^{\mathrm{r} 36}$ ) guidarono a nuove costruzioni pel centro di curvatura in un punto d'una tale curva, supposta determinata dalla relativa tangente e da tre nuovi punti ${ }^{\mathbf{3}}{ }^{7}$ ), altre [I8I] diedero risultati congeneri ed ebbero per punto di partenza questo teorema di CHASLES ${ }^{\mathbf{1 3 8}}$ ): se si fa ruotare una trasversale attorno ad un punto $O$ del piano d'una conica e per ogni sua posizione si determina la projezione su di essa del polo relativo, si ottengono tutti $i$ punti d'una parabola. Delle costruzioni pel centro di curvatura, differenti da quelle ora citate, si apprendono da una delle ultime pubblicazioni [222] del nostro Autore ed altre ancora in un'elegante Memoria [203] consacrata alle applicazioni ed allo svolgimento di un nuovo elemento introdotto da M. D'OCAGNe ${ }^{\text {139}}$ ) nella teoria delle coniche. Tutti questi lavori, benchè modesti in apparenza, al pari dei precedenti, sono riboccanti di osservazioni geniali ed utili e di costruzioni di suprema eleganza.

Quadriche. - Mentre coi lavori testè discorsi il Mannheim prese posto nell'innumerevole schiera degli epigoni di Apollonio Pergeo, con quelli di cui ora ci proponiamo di dire qualche cosa egli ha conquistata una posizione eminente nel drappello, relativamente esiguo, di coloro che sforzaronsi di far raggiungere alla teoria delle quadriche un grado di perfezione comparabile a quello da tempo conseguito dalla teoria delle coniche. Tale aspirazione sembraci palese in un lavoro [57] ove egli, dopo di avere confermata la generabilità di qualunque curva di second'ordine col mezzo di due fasci di raggi fra loro projettivi, considera una quadrica passante per sei punti $A, B, C, P^{\prime}$, $P^{\prime \prime}, P^{\prime \prime \prime}$. Se $P$ ne è un altro punto arbitrario e si chiamano $r, r^{\prime}, r^{\prime \prime}$ i birapporti delle quaterne di piani projettanti dalle rette $B C, C A, A B$ i punti $P, P^{\prime}, P^{\prime \prime}, P^{\prime \prime \prime}$, avrà luogo una relazione della forma

$$
B r r^{\prime}+C r r^{\prime \prime}+D r^{\prime} r^{\prime \prime}+E r+F r^{\prime}+G r^{\prime \prime}=0
$$

134) Cfr. [I52], Pp. II5-118, I21-122, I64; inoltre [219].

135) Note on the geometry of conics [The Messenger of Mathematics, $\mathrm{II}^{e}$ serie, t. XII (I882), p. 40-4I].

${ }_{136}$ ) Cfr. [184], pp. 578-582.

x37) Cfr. anche la Questione 1960 delle Nouvelles Annales de Mathématiques [IV série, t. III (1903), p. 48], proposta dal Mannhem e da lui risolta [Ibid., id., pp. 476-479].

${ }^{18}{ }^{8}$ ) Traité des sections coniques, I ère Partie (Paris, I865), p. I45.

139) De la déviation dans l'ellipse [Nouvelles Annales de Mathématiques, IIIème série, t. V (I886), pp. $370-380]$. 
(ove le sei costanti $B, C, \ldots, G$ sono legate da due relazioni), la quale viene dal Mannheim considerata per l'equazione di una quadrica contenente $\mathrm{i}$ sei punti dati: imparzialità storica esige si avverta come l'utilità di essa non venne sino ad ora dimostrata nè dal Nostro, nè da altri.

Un decennio più tardi egli, dalle sue ricerche intorno alla superficie delle onde ${ }^{\mathrm{r} 4^{\circ}}$ ), fu condotto [134] alla ricerca del legame esistente fra i raggi principali di curvatura in un punto comune a tre quadriche omofocali e la compi partendo dalla seguente proprietà da lui anteriormente scoperta: Se itn angolo di grandezza costante circoscritto ad un ellissoide $(E)$ sta in un piano normale all'ellissoide nei due punti di contatto dei lati dell'angolo ed ha per vertice un punto d'un ellissoide $\left(E_{1}\right)$, il suo vertice descriverà una linea di curvatura di $\left(E_{1}\right)$. Servendosi poi di due semplicissime proposizioni di Geometria elementare egli ritrova un noto teorema di LAMÉ e giunge a molti nuovi risultati, fra cui citiamo i seguenti: Il raggio di curratura in un punto $P$ della sexione prodotta in un ellissoide da un piano normale in $P$ ad una linea di curvatura è proporzionale al segmento di quel raggio compreso fra $P$ e la sua intersezione con un piano principale dell'ellissoide. Le normali condotte ad un ellissoide dai punti di una linea di curvatura sono divisi in parti proporzionali dai piani polari degli stessi punti rispetto agli ellissoidi omofocali al dato, ecc.

Il Mannheim ha poi trovate molte notevoli proprietà [135], le quali sembrano destinate a formar parte di qualunque futura teoria completa delle quadriche omofocali; riferiamo a mo' d'esempio la seguente: Sia $m$ un punto comune a tre quadricbe omofocali; $N, N^{\prime}, N^{\prime \prime}$, le relative normali; $a b c, a^{\prime} b^{\prime} c^{\prime}, a^{\prime \prime} b^{\prime \prime} c^{\prime \prime}$ le terne di punti in cui queste normali intersecano $i$ piani principali del dato sistema; sia $\alpha$ il punto comune a piani condotti da a, $a^{\prime}, a^{\prime \prime}$, normalmente risp. a $N, N^{\prime}, N^{\prime \prime} ; \nLeftarrow e \gamma$ abbiano analogbi significati. I tre punti $\alpha, \beta, \gamma$, appartengono alla stessa retta $\Delta$, le cui projezioni sopra i piani $N^{\prime} N^{\prime \prime}, N^{\prime \prime} N, N N^{\prime}$ tagliano quelle normali nei centri principali di curvatura delle tre quadriche nel punto $\mathrm{m}$.

Questa proposizione si ritrova, e se ne vedono le svariate conseguenze, in uno scritto di poco posteriore [136], dal quale si apprende la soluzione geometrica della questione, già risoluta analiticamente dal LAGUERRE ${ }^{\mathrm{I} 4 \mathrm{r}}$ ), di determinare in un punto qualunque d'una quadrica gli assi della relativa indicatrice ed $i$ centri principali di curvatura ${ }^{142}$ ); di essa il Nostro diede poi [197] una seconda soluzione ancora più

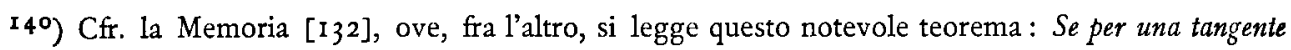
ad una linea di curvatura d'un ellissoide si conducono i piani tangenti ad un ellissoide omofocale, l'angolo che essi formano dipende, non dalla tangente, ma soltanto dalla linea di curvatura scelta.

${ }^{{ }^{4} \mathrm{r}}$ ) Sur la détermination, en un point d'une surface du second ordre, des axes de l'indicatrice et des rayons de courbure principaux [Journal de Mathématiques pures et appliquées, III ${ }^{\mathrm{e}}$ série, t. IV (I878). pp. 247-256. - Euvres de LAGUerRe, t. II (Gíométrie) (Paris, I905), pp. 520-529].

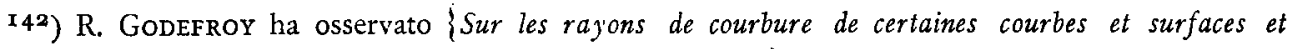
en particulier des courbes et des surfaces de LAMÉ [Journal de l'École polytéchnique, LXIV cah. (1892), p. 44]\} che la costruzione del MannheIm per l'indicatrice in un punto di una quadrica è applicabile a tutte le superficie rappresentabili con equazioni del tipo $a x^{m}+b y^{m}+c z^{m}+d=0$. 
semplice ed elegante, anzi di semplicità che sembra ulteriormente irriducibile e di eleganza da giudicarsi insuperabile.

Citiamo di sfuggita una breve Nota [I 40], che si direbbe un prodotto secondario degli studi del Mannheim sulle quadriche omofocali e che non andrebbe trascurata da chi intendesse redigere una completa bibliografia della strofoide; ed osserviamo piuttosto come a lui debbansi delle argomentazioni abbastanza semplici per determinare le condizioni affinchè le altezze d'un tetraedro giacciano sopra una quadrica di rivoluzione ${ }^{\mathbf{1} \mathbf{4}^{3}}$ ) e per dimostrare geometricamente essere un'iperbola il meridiano della superficie generata dalla rotazione di una retta attorno ad altra che non l'incontra ${ }^{\mathbf{x} 4}$ ).

Ad occuparsi delle superficie di second'ordine egli fu ricondotto dalla Memoria di P. ADAm: Sur l'équation d'Euler et sur les lignes de courbure de l'ellipsoide ${ }^{\mathbf{x} 5}$ ); ivi sono considerate delle coppie di ellissoidi le cui linee di curvatura si corrispondono con parallelismo dei loro piani tangenti; ora il Mannherm, col mezzo di considerazioni della massima semplicita, ha mostrato [189] che dette linee si corrispondono anche con parallelismo delle loro rette tangenti e che inoltre i piani delle sezioni circolari delle due superficie sono fra di loro paralleli : se, viceversa, quest'ultima circostanza si verifica, $\mathrm{i}$ due ellissoidi si trovano nella posizione considerata dall'ADAM.

Un'ultima osservazione ci resta da riferire. PonCELET ${ }^{146}$ ) ha asserito che, se un quadrilatero è circoscritto ad una quadrica, $i$ punti di contatto de' suoi lati stanno in un piano. Orbene il Nostro ha giustamente notato [202] che tale enunciato è troppo assoluto, esistendo altri quadrilateri circoscritti non soddisfacenti alla suindicata condizione. Ciò è in pieno accordo con le risultanze di anteriori scritti di G. Bruno, H. G. Zeuthen ed A. Voss ${ }^{147}$ ), di cui egli non ebbe certamente notizia; perciò l'originalita di tali indagini si limita al metodo usato, il quale consiste nella trasformazione in una sfera della quadrica considerata e nella successiva applicazione di un'opportuna inversione.

\section{Geometria descrittiva.}

Nell'ultima faccia dell'attività scientifica del Mannheim si rispecchia la sua azione come insegnante ${ }^{{ }^{148}}$ ). Appunto nel corso delle sue lezioni gli venne fatto di osservare ( $[\mathbf{I} 37],[138]$ ) come nelle tavole di Geometria descrittiva fosse, non solo possibile e lecito, ma anche utile, di prescindere dalla posizione della linea di terra, tenendo conto soltanto

I43) L'Intermédiaire des Mathématiciens, t. II (I895), pp. I I I-I I 2.

I44) L'Intermédiaire des Mathématiciens, t. IX (I902), pp. 287-288.

I45) Bulletin de la Société Mathématique de France, t. XXII (I894), pp. 205-208.

r46) Traité des propriétés projectives des figures, t. I, p. 48.

147) Cfr. LoRIA, opera citata II5), p. 97.

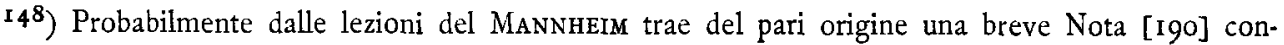
tenente la dimostrazione di un teorema, naturale estensione di uno notissimo ed utilissimo in Geometria descrittiva; eccone l'enunciato: Se due o piu curve tracciate sopra una superficie $(S)$ banno fra loro, in un punto a, un contatto dell'ordine $n$, le loro projezioni da un punto qualunque sopra di un piano arbitrario banno fra loro un contalto d'ordine $n+\mathrm{I}$ nel punto $a^{\prime}$ projezione di a. 
della sua direzione: cosi facendo, i relativi disegni acquistano in generalitd senza perdere in esattezza o perspicuità. Per tradurre in atto tale concetto è indispensabile di avere a propria disposizione delle soluzioni dei problemi relativi a piani, le quali siano indipendenti dalle tracce dei piani stessi; siffatti nuovi procedimenti vennero indicati dal Nostro. La bontd della suindicata tesi e la forza degli argomenti usati per stabilirla sono dimostrate da ciò che le idee del Mannherm vennero favorevolmente giudicate, non solo in Francia, ma anche nel Belgio, in Russia, in Portogallo; ed il fatto, che in nessuno dei nostri corsi universitari manchi un cenno della determinabilità di un piano col mezzo di elementi diversi dalle tracce, prova che anche in Italia esse vennero accolte col meritato favore.

Della soppressione della linea di terra il Mannheim non ebbe occasione di fare menzione esplicita nell'ottima opera [I 27], [152 ${ }^{149}$ ) scritta col duplice scopo di fungere come testo per le sue pubbliche lezioni e di diffondere i suoi metodi d'insegnamento al di fuori della cerchia dei suoi ascoltatori e che stabili nel modo più saldo la sua fama di maestro capace di essere conciso e riuscire chiaro; non ebbe occasione che di servirsene per incidenza, perchè egli suppose (come aveva, non il diritto, ma il dovere di fare, data l'organizzazione della Scuola politecnica) i suoi scolari gid famigliari col metodo di Monge. Dedicó, in conseguenza, la I $\mathrm{I}^{\mathrm{a}}$ Parte del suo Cours (Studio dei vari modi di rappresentazione dei corpi) ai metodi di Geometria descrittiva diversi dalla doppia projezione ortogonale ed al loro uso nel tracciamento delle ombre; tali metodi sono quello dei piani quotati, la prospettiva, la projezione cavaliera e l'assonometria, con speciale riguardo all'isometria. Quanto è indispensabile per servirsene viene dal provetto insegnante esposto con concisione euclidea, ma con la lucidita caratteristica della scuola matematica francese; viene poi applicato ad esempi interessanti consigliati, sia dal loro intervento nell'architettura (arcate, nicchie, ecc.), sia dalle svariate conseguenze geometriche a cui conducono: limitiamoci a segnalare gli sviluppi (pp. I I3-I I8, I2I-I22) relativi alla costruzione degli assi d'un'ellisse determinata da una coppia di diametri conjugati (cfr. [139]) e (p. I25) alcune nuove proprietà delle sezioni coniche (cfr. [218]).

Originalità assai maggiore presenta la $\mathrm{II}^{2}$ PARTE del Cours in questione (Curve $e$ superficie, complementi teorici.ed applicazioni). Ivi l'Autore, profittando della maggiore libertà concessa nel I867 agli insegnanti dal Consiglio di perfezionnamento della Scuola politecnica, si propose di mostrare come la Geometria cinematica porgesse mezzi possenti per risolvere con uniformità, semplicità ed eleganza, e senza chiedere l'ajuto dell'Analisi, tutte le questioni essenziali relative a curve e superficie. Del largo uso che egli fece dei propri lavori precedenti il lettore ebbe già implicita notizia dai numerosi

149) Queste due edizioni non differiscono che per alcuni miglioramenti nei particolari, mentre il piano generale è lo stesso. Nel nostro resoconto noi ragioneremo, per ragioni evidenti, sulla $2^{\mathbf{a}}$. Nel presentare la $\mathrm{I}^{\mathrm{a}}$ all'Accademia dei Lincei, il Cremona ebbe per l'opera del Mannheim parole di vivo elogio, perchè egli la trovó possedere "al più alto grado quelle doti di perspicuità ed eleganza che raramente mancano in trattati francesi " [Atti della R. Accademia dei Lincei (I879-80), Transunti, vol. IV, p. 69]. 
richiami al Cours sparsi nelle pagine precedenti, richiami che ci dispensano dal tediarlo ora segnalando i punti di contatto fra il Cours stesso e le anteriori pubblicazioni del nostro geometra.

Prima di esporre il contenuto della $\mathrm{II}^{\mathrm{a}}$ Parte di esso, giova avvertire come da essa emerga nel modo più luminoso che il Mannheim apparteneva alla categoria che il PoinCARÉ chiama dei geometri intuitivi ${ }^{150}$ ); non si cerchino quindi nel Cours de Géométrie descriptive considerazioni pienamente rigorose o precise determinazioni del campo di validità di proposizioni o di metodi; in qualunque punto di una delle curve o superficie considerate viene tacitamente postulata l'esistenza di rette o piani tangenti od osculatori, onde tali enti geometrici si ammettono essere sempre quali l'ordinaria Geometria insegna a generare e quali vengono offerti dalle applicazioni: resta quindi intatta la questione dei limiti di applicabilità dei risultati ottenuti, questione certamente attraente ed importante, che il Nostro autore non ha nemmeno enunciato, pago di avere escogitate metodi bellissimi e degni di fiducia amplissima, se anche non sconfinata.

La segnalata attitudine del MANNHEIM manifestasi sotto luce meridiana sin dalle prime pagine di quella $\mathrm{II}^{\mathrm{a}}$ PARTE, ove le generalità sulle curve e sulle superficie vengono semplicemente enunciate, senza schiettamente distinguere quelle provenienti dalla semplice intuizione, da quelle che rappresentano il risultato delle prime applicazioni dell'Analisi alla Geometria. Seguono tosto le definizioni e le proposizioni più essenziali concernenti il movimento di una figura piana nel proprio piano, con interessanti applicazioni all'ellisse, all'epicicloide $c$ ad altre curve. Passando allo spazio, vengono anzitutto considerate le curve gobbe, con speciale riguardo all'elica cilindrica, e poi le superficie rigate: ma lo studio di queste viene provvisoriamente interrotto, per essere ripreso ed esaurito dopo l'esposizione delle proprietà fondamentali dei movimenti nello spazio e l'applicazione degli stessi alla teorica della curvatura delle superficie. Ritornando alle superficie che contengono $\infty^{\mathrm{t}}$ rette, il Mannheim investiga esaurientemente, dal punto di vista della Geometria descrittiva, le superficie elicoidi rigate, arrestandosi con visibile compiacimento sulle superficie delle viti a filetto triangolare o quadrangolare: la trattazione, pure senza perdersi in particolari superflui, ha tutta la desiderabile ampiezza; le costruzioni esposte sono, in parte originali, ma tutte elegantissime. Seguono due sostanziosi capitoli sulle superficie sviluppabili (in particolare su quelle di eguale pendenza) e sulle rigate in generale ${ }^{\mathbf{I} 5}$ ), a cui seguono nuovi sviluppi di Geometria cinematica. L'ultimo argomento trattato puó dirsi un complemento a quanto nella $I^{a}$ PARTE si espose intorno ai piani quotati, dal momento che si riferisce alle superficie topografiche.

In questi rapidi cenni abbiamo dovuto forzatamente passare sotto silenzio buon nu-

150) Vedi la conferenza: Du rôle de l'intuition et de la logique en mathématiques [Compte rendu du II Congrès International des Mathématiciens (Paris, Gauthier-Villars, I902), pp. II5-I30].

$\mathbf{5}^{\mathbf{I}}$ ) Sembraci opportuno di attrarre qui l'attenzione del lettore sulla seguente questione di cui il Nostro asseri di possedere due soluzioni: Una retta costantemente tangente a tre superficie genera una rigata; costruire la tangente alla curva di contatto di essa con una delle superficie date [L'Intermédiaire des Mathematiciens, t. VI (I899), p. I24]. Vedi anche Ibid., t. IX (I902), p. 322, Question 2498. 
mero di cose interessanti adunate nell'opera in questione (per esempio i molteplici sviluppi relativi al tracciamento delle ombre e le innumerevoli proprieta di curve e superficie particolari); lo facemmo, e perchè ci sentiamo sospinti dalla lunga via, e perchè il Cours in questione, in venticinque anni di rita, consegui già grande notorietà. Tuttavia, prima di volgerci ad altro argomento, vogliamo soggiungere due osservazioni; e cioè che mediante tale opera il Mannherm ha luminosamente dimostrato che i cultori della Geometria descrittiva possono fare sicuro assegnamento sulla Geometria cinematica come prezioso metodo di scoperta di costruzioni convenientissime, come ausiliare agile e vigoroso, e che le numerose citazioni sparse a larga mano in tutto il Cours fanno fede della vasta coltura, della solida erudizione del Mannheim, cose di cui forse taluno poteva essere tentato di dubitare considerando l'impronta personale e la rigorosa unità (confinante con l'unilateralitì) di tutta la sua produzione scientifica.

\section{III. - Un Autunno rimuneratore.}

Il vasto campo coltivato dal Mannheim con geniale perseveranza si era mostrato tanto fecondo che, circa quarant'anni dopo di avere cominciato a dissodarlo, egli giudicò fosse giunto il momento di falciare la messe omai biondeggiante e trasformarla in vitale nutrimento per le generazioni venture.

Il progetto di erigere in un corpo di dottrina la Geometria cinematica aveva già lusingato il Bobillier, che avevalo a lungo accarezzato e poi abbandonato solo quando la gelida ala della morte lo aveva colpito ${ }^{\mathbf{1 5 2}}$ ). Il Mannheim stesso ne aveva misurata l'importanza sino dagli esordi della sua carriera ${ }^{\mathrm{r} 3}$ ) e vi aveva dato un principio di attuazione col suo Cours de Géométrie descriptive; ora l'effettuazione di esso è rappresentato da un poderoso volume [184], destinato a documentare $\mathrm{i}$ suoi diritti a venire considerato siccome il primo legislatore di una nuova e bella provincia di cui, in parte per merito suo, erasi arricchito il regno della Geometria; in esso trovansi coordinati o riassunti, talora anzi semplicemente riprodotti, i principali dei suoi lavori geometrici : tale natura dell'opera che ora ci apprestiamo ad analizzare spiega le molteplici citazioni che di essa facemmo nelle pagine precedenti e risultera meglio da quanto segue.

I Principes et développements de Géométrie cinématique constano di tre parti ed un'Appendice.

La I $\mathrm{I}^{\mathrm{a}}$ Parte concerne la Geometria del piano, con un cenno finale sulla Geometria sferica, la $\mathrm{II}^{\mathrm{a}}$ la Geometria dello spazio, mentre nella $\mathrm{III}^{\mathrm{a}}$ sono raccolte svariate applicazioni.

Definita la Geometria cinematica, come dottrina dei movimenti indipendente dalla considerazione delle forze e del tempo, e lo spostamento (déplacement) come movimento indipendente dalla considerazione della velocità, il Mannheim espone nella I ${ }^{\mathrm{a}}$ Parte della

152) Veggasi a questo proposito un'interessante Nota storica del Nostro nel L'Intermédiaire des Mathématiciens, t. VIII (I 901 ), p. 330.

153) Cfr. la chiusa della Memoria [14]. 
sua opera le più essenziali proprietà del centro d'istantanea rotazione ed il conseguente metodo per costruire le normali ad una estesa classe di curve: la fecondità di tale metodo risulta da molti esempi, alcuni dei quali desunti da lavori precedenti ([9o], [I 20], [12I]). Si passa poi allo studio del movimento in un piano d'un triangolo variabile ${ }^{\mathbf{5 4}}$ ), con applicazioni (cfr. [Io], [I2], [I4]) alla ricerca dei centri di curvatura di alcune linee piane, ed alle analoghe questioni (cfr. [13]) relative alle linee descritte od inviluppate da punti o linee appartenenti ad una figura piana moventesi comunque nel proprio piano, Le seguenti ricerche sugli spostamenti di un poligono piano conducono il MANNheim a formole generali, che il Bour inseri nel $1^{\circ}$ Fascicolo del suo Cours de Mécanique et Macbines professé à l'École polytechnique (Paris, I865), e di cui qui si veggono molte belle applicazioni alla costruzione di normali e centri di curvatura (cfr. [14], [27], [28]), alla teoria delle caustiche piane [I60] ed alla dimostrazione delle proprietà dei più noti sistemi articolati (cfr. [90], [I50], [205]). La I ${ }^{a}$ PARTE dell'opera in questione si chiude con la riproduzione del lavoro [26] sulle curve piane e sferiche considerate come inviluppi di cerchi, riproduzione fatta per porgere il destro all'Autore di porre $i$ fondamenti della Geometria cinematica sferica.

Nella II ${ }^{a}$ PARTE ritroviamo anzitutto (pp. 97-I 26), con pochi ritocchi di forma e qualche nuova applicazione alle superficie rigate ed alle elicoidi, la fondamentale Memoria del Mannheim sul movimento infinitesimo d'un solido [48], su cui è superfluo ritornare ora; altrettanto faremo riguardo alle pagine seguenti (pp. I27-I4I) che nulla apprendono a chi conosce altri scritti ([4I], [II8], [II9]) del Nostro. In tal modo egli ha adunato tutti i materiali per erigere una teoria geometrica della curvatura delle superficie; ad essa sono consacrate le pp. I4I-I60, nelle quali si ritrovano raccolte in bụon ordine, e con le aggiunte rese necessarie dalla logica concatenazione, le proposizioni contenute in Memorie a noi già note ([58], [6o], [64], [77], [78], [82]).

Ritornando alla Geometria cinematica, il Mannheim si volge allo studio di movimenti per qualche ragione specializzati, cominciando dal moto d'una retta [68]; ed introducendo poi la condizione [164] che tutti i punti di essa descrivano delle ellissi, și procura gli elementi per l'indagine del movimento d'una figura rigida di cui ogni punto descriva una linea di second'ordine. Seguono poi le ricerche, di cui gia facemmo cenno ([I68]-[170]), sul moto di una retta di cui quattro punti descrivono altrettante sfere con $\mathrm{i}$ centri coplanari, dalle quali ricerche egli assurge a considerazioni generali sul movimento di una retta di cui quattro punti sono soggetti a descrivere ciascuno una superficie data ${ }^{\mathbf{1 5 5}}$ ). Ritroviamo poi i risultati dello studio [148] del movimento di una retta, tre punti della quale stanno sulle facce d'un triedro trirettangolo e quindi la teotia

154) Per l'analogo moto nello spazio vedi la Quegtione I 3 I proposta dal Nostro nel L'Intermédiaire des Mathématiciens, t. I (I894), p. 8I.

155) Veggasi anche la soluzione data dal Nostro alla Questione 998 del L'Intermédiaire des Mathematiciens [t. IV (1897), pp. 2[0-24 1 ]. 
(cfr. [153]-[156]) dell'iperboloide articolato ${ }^{\mathrm{r} 56}$ ) e le proprietà essenziali della poloide e dell'erpoloide. Pure a noi già note ( $\mathrm{Cfr}$. [ $\mathrm{I}_{42}$ ) sono le successive investigazioni intorno al moto di un diedro rigido e di un fascio di piani, nonché le proposizioni risultanti, che mettono in luce l'analogia fra la Geometria cinematica di una punteggiata e quella di un fascio di piani (cfr. [73], [8I], [179]): rileviamo la costruzione che se ne trae per l'iperboloide osculatore lungo una generatrice all'iperboloide definito da tre curve o superficie direttrici. Relazioni utili provengono dal supporre infinitesimo il moto del diedro considerato; applicandole al caso in cui le facce del diedro siano le sezioni principali in un punto qualunque di una superficie si ottiene una teoria completa del paraboloide delle otto rette ${ }^{157}$ ). Il Mannherm vi riattacca lo studio ([100], [102]) delle superficie i cui raggi di curvatura sono funzioni l'uno dell'altro ed alcune osservazioni concernenti il movimento di due notevoli triedri trirettangoli, cioè il triedro principale di una curva gobba ${ }^{\mathbf{5} 58}$ ) e il triedro avente per spigoli la normale in un punto ad una superficie e le tangenti ivi alle corrispondenti linee di curvatura.

Dopo alcune considerazioni dotate di più vasta portata (pp. 240-245) sul movimento di una figura rigida soggetta a condizioni multiple, ritroviamo (pp. 246-258) l'essenza delle ricerche del Mannherm [9I] sul moto d'un solido soggetto a quattro condizioni, uno studio (pp. 258-268) della conoide di PLÜCKER, considerata nei suoi rapporti con la Cinematica (cfr. [I59]) e, dopo un cenno della rappresentazione piana degli spostamenti di una figura rigida con due gradi di libertà (cfr. [I44]), la teoria dei pennelli di raggi e delle normalie [64]. Come epilogo il Nostro autore traccia le prime linee d'una teoria del movimento d'un solido soddisfacente a tre sole condizioni.

Nella III a Parte, sotto il titolo di Applicazioni diverse, il Mannheim raccoglie le risultanze di indagini di cui già parlammo: costruzione del centro di curvatura dell' ellisse di CASSINI $\left[\mathrm{I}_{7} 6\right]$; proprietà delle normali a superficie algebriche e in particolare alle quadriche [75]; teoria delle superficie rigate [II3]; contatti di terz'ordine delle superficie [6I]; soluzioni geometriche di problemi che concernono le superficie e dipendono da infinitesimi del terz'ordine [84]; proprietà delle superficie parallele [133]; costruzione del centro di curvatura dell'evoluta del contorno apparente d'una superficie projettata ortogonalmente su di un piano [165]; teoria geometrica delle curve di BerTRAND [66]; metodo di trasformazione in Geometria cinematica ([168]-[170], [179]); moto di una figura rigida di cui tutti i piani passano per altrettanti punti fissi [173]; superficie delle onde; movimento del doppio cono ([I I I ], [I 72$]$ ). Chiudono questa $\mathrm{III}^{\mathrm{a}}$ Parte alcune ricerche inedite, che risalgono al I870, sul movimento infinitesimo d'un poliedro variabile (pp. 457-475) ed aventi lo scopo di generalizzare allo spazio i risultati di studi precedenti sulla deformazione d'un poligono. L'utilità delle formole con-

I56) Cfr. una Nota storica del Mannheim nel L'Intermédiaire des Mathématiciens [t. IV (I897), p. 93].

157) Per un complemento veggasi [196].

${ }^{158}$ ) Un'aggiunta a questo passo dei Principes trovasi nella Nota posteriore [198]. 
seguenti emerge dai molti ed importanti problemi di cui esse porgono la soluzione; citiamo quelli che ci sembrano i più cospicui:

Una retta si muove con la condizione che quattro suoi punti descrivano altrettante superficie: costruire l'asse di curvatura della trajettoria descritta da un suo punto arbitrario. Sopra una rigata $(G)$ sono tracciate due curve $(m),(n)$ : costruire il piano normale in un punto della curva luogo dei punti che dividono il segmento $m n$ in un dato rapporto. Una retta tangente in $a, b$ a due superficie è limitata in $m, n$ a due altre: costruire la normale alla superficie luogo dei punti che dividono il segmento $m n$ in un dato rapporto. Ecc.

$\mathrm{Da}$ questo arido schema delle tre parti costituenti l'opera in discorso si scorge come in esse abbiano a trovar posto quasi tutte le ricerche del Nostro; alcune altre si incontrano nell'Appendice ove sono in primo luogo riprodotti i suoi primi lavori di Geometria cinematica ([Io], [ I 2], [14]), le sue belle indagini sul luogo delle posizioni successive dei centri di curvatura d'una linea che ruzzola sopra una retta [15] e sulla lunghezza comparata degli archi di curve differenti [30]; sono poi additate alcune proprietà delle spirali sinusoidi e di altre linee ad esse collegate [97], un modo speciale di rappresentazione delle superficie rigate ([I05]-[I07], [II2]) ${ }^{\mathrm{I} 59}$ ), alcuni teoremi sulle curve di Bertrand [i 49] e sulla superficie delle onde [i I6] e delle eleganti costruzioni pel raggio di curvatura d'una conica ([I74], [182]); da ultimo si rilegge la grande Memoria [I 43] che il Nostro consacrò all'Ottica geometrica.

Con tale Appendice il Mannherm fin' per rendere l'opera testè compendiata un quadro fedele e completo della propria produzione scientifica: come tale essa possiede un valore indiscutibile, grande, permanente. Non v'ha dubbio peró che se egli avesse avuto il coraggio di omettere qualche lavoro secondario e di operare in altri alcuni tagli, apportandovi qualche modificazione di forma, avrebbe tolto alla sua grande pubblicazione l'apparenza di mosaico sotto cui in parte si presenta e vi avrebbe dato sostanza e forma di esposizione veramente metodica della Geometria cinematica. Nè va taciuto come la stessa pubblicazione ponga in evidenza essersi il MANNHEIM consacrato toto corde a questa disciplina, non già come ad uno dei rami della teoria dei moti e delle forze, ma sibbene come ad un'ausiliare preziosa nella scoperta dei fenomeni offerti dall'estensione figurata: infatti, mentre essa è, riguardo alle applicazioni a curve e superficie, di una ricchezza imponente, per converso non contiene tutti i concetti essenziali nello studio geometrico dei movimenti; ad esempio in essa cercherebbesi indarno il fecondo principio dell'inversione dei movimenti, una delle più geniali scoperte di Chasles, a cui, ad esempio, A. Schoenflies assegnò una parte fondamentale in una sua ben nota esposizione della geometria cinematica ${ }^{\mathbf{1 6 0}}$ ).

Un certo numero di aggiunte ai Principes et développementes si leggono in alcuni brevi lavori, alcuni incidentalmente ticordati più sopra, altri da esaminarsi ora.

159) Cfr. la soluzione data dal Mannheim per la Questione I730 del L'Intermédiaire des Mathématiciens [t. VII (1900), p. 228].

I6 o) Geometrie der Bewegung in synthetischer Darstellung (Leipzig I886). 
Occupiamoci anzitutto di quelli contenenti ulteriori sviluppi ed applicazioni dei metodi suggeriti per la ricerca delle normali e dei centri di curvatura alle linee piane. In uno [199] è risoluta geometricamente la seguente questione, proposta dal Duporce: Per un punto arbitrario di una curva $C$ si conduca una retta $r$ formante con una direzione assegnata un angolo eguale a quello fatto con quella direzione dalla corrispondente tangente di $C$; costruire il punto di contatto di $r$ col proprio inviluppo. In due altri ([210], [2II]) leggesi una trattazione cinematica esauriente delle proprietà delle curve piane tali che le loro normali staccano da una retta fissa segmenti proporzionali agli archi descritti dai relativi punti $d^{\prime}$ incidenza ${ }^{\mathrm{x} 6 \mathrm{r}}$ ). In un quarto [217] ci basti segnalare la costruzione del centro di curvatura dell'inviluppo di una retta che intercetta sopra una curva data un arco di lunghezza costante.

Una terna di brevi Note del Mannheim si riferisce al problema seguente: dati sulla normale $A$ in un punto a d'una superficie $(S)$ i centri di curvatura della curva di contorno apparente di $(S)$ relativi a tre direzioni differenti dei raggi visuali, determinare gli elementi principali di curvatura di $(S)$ nel punto a. Una soluzione risulta da una proprietà dell'indicatrice di una superficie che il Mannherm enunciò [i86] e poi dimostró in parecchi modi ; mentre una seconda ([185], [191]) è corollario d'un teorema relativo alla conoide di PLÜCKer che completa un passo (p. 269) dei Principes et développements.

Alla Geometria infinitesimale, non delle superficie, ma delle curve sghembe, appartiene un lavoretto [201], la cui importanza non deve commisurarsi dalla mole, nel quale trovansi dimostrate geometricamente le formole di FrENET: se non erriamo, è questo il primo e ben riuscito tentativo per introdurre tali formole in una trattazione sintetica delle curve sghembe.

Per rendere completa l'enumerazione dei contributi del Mannherm alle varie branche della Geometria, fa mestieri che accenniamo, finendo, ad alcuni brevi scritti che, verso il tramonto della sua vita, egli dedico alla Geometria elementare e che pubblicó in parte pseudonimi, indubbiamente perchè vi annetteva scarsa importanza: uno [193] contiene una semplice dimostrazione della relazione approssimata

$$
\pi+0,0047=\sqrt{2}+\sqrt{3}
$$

un altro [204] concerne la curva, composta di archi circolari fra loro raccordati, che gli architetti francesi chiamano anse de panier; mentre i rimanenti ([2 I2], [214], [220][224]) concernono la Geometria del triangolo e provano come anche il Mannheim siasi lasciato trascinare da una notissima corrente di idee.

161) Cfr. gli articoli di Piccrol. (Sur les bélices cylindriques dont les normales principales rencontrent une droite fixe) e Duporce (Remarque sur la Note précédente) nelle Nouvelles Annales de Mathematiques, IVème série, t. II (1902) Pp. 177-181 e I-81-I84. 


\section{IV. - Pax!}

Il numero e l'importanza delle scoperte compiute dal Mannherm in tutta la Geometria ${ }^{\mathbf{6} 2}$ ) ne diffusero la rinomanza in tutto il mondo scientifico ${ }^{\mathrm{I} 63}$ ). Sino dal ${ }^{8} 872$ l'Istituto di Francia conferivagli il premio Poncelet pel complesso delle sue pubblicazioni ${ }^{64}$ ); più tardi un grande numero di sodalizi scientifici gareggiarono nel rendergli omaggio e sarebbe appunto questo il momento di enumerare tutte le Società ed Accademie che reputaronsi onorate chiamandolo nel proprio seno; ma ce ne asteniamo perchè, ove lo facessimo, ci parrebbe di recare un postumo oltraggio alla fiera modestia di chi, in qualunque occasione, si fregiò esclusivamente dei titoli a cui davagli diritto il suo posto nell'esercito francese ${ }^{\text {I65) }}$ ) e la sua posizione nel pubblico insegnamento. Tuttavia, di uno speciale ricordo è meritevole la cerimonia senza precedenti fatta nell'Aula magna della Scuola politecnica il I4 dicembre I90I, quando egli, colpito dall'inesorabile legge sopra i limiti d'età, fu costretto a scendere dalla cattedra dalla quale aveva educato al sano geometrizzare trentotto generazioni di studenti, circa novemila discepoli, costituenti il fiore della gioventù francese ${ }^{166}$ ).

Fu questa l'ultima gioja che la sorte concesse al valoroso geometra!

Crudelmente colpito dalla morte d'una figlia diletta, egli, al pari del poeta de Les contemplations in circostanze luttuosamente analoghe non chiese più

A la création immense

Qu'un peu de silence et de paix.

Strappato a forza dalla sua cattedra, egli non abbandonó la ricerca scientifica ${ }^{\mathbf{1 6} 7}$ ), ma nell'ultima fase della propria esistenza si dedicò con maggiore impegno a quelle opere di carità, che non erano mai rimaste estranee al programma della sua attivitd; cosi, per un'altra ragione, un rimpianto unanime segui la notizia della sua morte, avvenuta in Parigi l's I Dicembre 1906: che se tale sentimento non ebbe clamorose ma-

162) Giova qui notare che la sua capacità di maneggiare l'Analisi è attestata dalle Solutions of two Questions (5492, 5544) proposte da Hermite [Educational Times, Vol. XXIX (1878), pp. 76-79].

r63) Cfr. le numerose citazioni di lavori del Mannherm che trovansi nell'articolo Kinematik scritto da A. Schoenflies per l'Encyklopädie der Mathematischen Wissenschaften, Bd. IV ${ }_{1}$ (Ig02). Gli scritti relativi alla Geometria della retta sono in parte ricordati da $\mathrm{K}$. Zindere nella sua relazione: Die Entwicklcong und der gegenwärtige Stand der differentiellen Liniengeometrie [Jahresbericht der Deutschen Mathematiker-Vereinigung, Bd. XV (I906), pp. I85-213].

164) Cfr. Comptes rendus hebdomadaires des séances de l'Académie des Sciences, t. LXXIX (2 $2^{\text {eme }}$ semestre 1874$)$, p. I 537 .

165) Egli raggiunse il grado di colonnello e fu insignito della commenda della legione d'onore.

r66) Particolari su tale cerimonia si leggono nell'articolo: Hommage rendu par l'École Polytechnique à Monsieur le Colonel Mannherm [Journal de l'École Polytechnique, II ème série, VIrème Cahier (I902), pp. 221-233]. Cfr. altresi: E. Duporce, Un bommage au Colonel Mannheim [Nouvelles Annales de Mathermatiques, IVème série, t. II (1902), pp. 25-27].

I67) Lo provano i lavori [210]-[224]. 
nifestazioni pari alla profondità ed universalità di esso, gli è che per volere dell'estinto nessun discorso venne pronunciato sulla sua tomba.

La fiera modestia, di cui questo ultimo desiderio è nuova ed impressionante attestazione, non dev'essere scambiata come scarsa considerazione del Mannheim per l'opera propria; giacchè sarebbe agevole raccogliere dai suoi scritti molte frasi dimostranti come egli ne misurasse esattamente l'alta importanza, sia dal punto di vista del metodo (cioè come contributo alla Geometria pura), sia come rappresentante un reale progresso nelle nostre cognizioni positive.

Tuttavia, riguardo al primo aspetto della propria produzione, egli non avrà tardato a riconoscere, con amarezza e dolore, come spento Chasles, non gli riuscisse di galvanizzare il corpo che, concepito da Euclide e ravivivato da Poncelet, sullo scorcio del secolo XIX sembrava affetto da paralisi progressiva. L'Analisi, riconquistata una nuova volta l'egemonia, attraeva ed assorbiva le forze migliori, consigliando e sospingendo coloro che sentivansi sedotti dell'eterno fascino della Geometria a coltivare la teoria degli enti algebrici, la cui creazione sard perenne titolo di gloria per quest'ultimo trentennio. A tale mirabile movimento il Mannheim si mantenne totalmente estraneo; dei relativi scritti egli s'interessava unicamente in quanto servivano di eccitanti al proprio pensiero, soltanto in quanto avessero virtù di suggerirgli nuove questioni, a cui applicare $i$ suoi metodi prediletti; ma essi mai ebbero la forza di allontanargli dalle labbra la coppa a cui (per usare una felice imagine di A. DE Musset) egli costantemente abbeveravasi. Ciò gl'impedi di fare sua propria l'opinione sempre più diffusa della necessità del rigore assoluto negli enunciati e nelle dimostrazioni delle proposizioni matematiche, gli fece lasciare nell'ombra le esatte determinazioni di segni nelle figure e di applicabilità dei teoremi e dei metodi, e gli vietó di prevenire le critiche di coloro che asseriscono essere tuttora sub judice qualunque proposizione la cui dimostrazione non sia in ogni sua parte inoppugnabile.

Ma se, lasciando in disparte le considerazioni di metodo, per loro natura temporanee, noi abbracciamo nel loro insieme la totalità delle questioni affrontate dal Mannheim, riconosceremo che, nel vasto oceano da lui solcato coraggiosamente in tutti i sensi, mai ebbe a patire l'onta d'un naufragio, sicchè l'orma da lui impressa sul cammino che guida alla verità è cosi vasta e profonda che il tempo non potrà cancellarla. Mentre se ci proponiamo di misurare la forza ed estensione degli strumenti che egli seppe foggiare, perfezionare od usare ci sentiremo infiammati di ammirazione e di fede nel loro avvenire, ricordando che "il sentiero dei giusti è come la luce che spunta, la quale va vieppiù risplendendo finchè sia chiaro giorno ${ }^{\mathrm{x} 68}$ ).

Alle pagine da lui scritte dovranno ricorrere, non soltanto il puro geometra, desideroso di apprendere soluzioni semplici ed eleganti e di toccare gli ultimi confini dell'odierno impero geometrico, ma indistintamente tutti coloro che agognano di conoscere completamente le qualita di forma che sono possedute dal nostro spazio. L'uno e gli altri

I68) Proverbio di Salomone. 
non tarderanno cosi a riconoscere che egli per primo riusci a piantare il vessillo vittorioso della scienza su molte vette ritenute dianzi inaccessibili, ricorrendo a veicoli che, se anche non possono giudicarsi di assoluta stabilità e completa sicurezza, sono del tipo di quelli che i pioneri della scienza sogliono usare con superba disinvoltura, per schiudere ai contemporanei regioni feconde, dianzi inaccessibili.

\section{ELENCO DELLE PUBBLICAZIONI MATEMATICHE DI A. MANNHEIM.}

\section{A V V B R T E X Z A.}

Nel presente Elenco (nel compilare il quale fui validamente assistito dal Ch. ${ }^{\text {mo }}$ Prof. Guccia, a cui esprimo la mia più viva gratitudine) non vennero incluse le numerose questioni proposte e risolute dal Mannheim nelle "Nouvelles Annales de Mathématiques », nel "L'intermédiaire des Mathématiciens », ed in altri periodici; alcune portano l'esplicita indicazione dell'autore, mentre di altri l'autore è indicato come "Ancien élève de Mathématiques spéciales " ${ }^{269}$ ) o col pseudonimo "CANON»; $i$ più interessanti fra tali scritti minori si trovano citati nel testo. Da notarsi che tutte le pubblicazioni del MaNNHEIM, anche se portano titoli in inglese, sono scritte in francese.

\section{848 .}

[I]. Solution géométrique du problème sur l'axe radical.

Nouvelles Annales de Mathématiques, Ière série, tome VII (1848), pp. 23 I-232.

[2]. Theorème sur les axes de l'ellipse et de l'hyperbole.

Ibid., id., id., p. 232.

\section{85 I.}

[3]. Note sur la Théorie des polaires réciproques. (Litogr.).

Metz, Imp. et lith. Nouvian, Janvier I85 I. (I3 pages).

[4]. Règle à calculs modifiee. Instruction.

Metz, Imp. et lith. Nouvian, Décembre I85 I.

\section{853 .}

[5]. Lieu des centres des circonférences coupant sous des angles égaux trois circonferences données.

Nouvelles Annales de Mathématiques, Ière série, tome XII (1853), pp. Ir3-II6.

I69) Fra queste trovansi (Nouvelles Annales) le soluzioni delle questioni proposte nel periodo I879-1886 per l'ammissione alla Scuola politecnica. 
[6]. Règle d'calculs modifiée.

Ibid., id., id., pp. 327-329.

\section{6.}

[7]. Théorème segmentaire sur le triangle.

Ibid., id., tome XV (1856), p. $60^{170^{\circ}}$ ).

[8]. Limaçon de Pascal.

Ibid., id., id., p. 289.

\section{857.}

[9]. Transformation des propriêtés métriques des figures à l'aide de la théorie des polaires réciproques.

Un volume in-8., de pages xix-59, avec figures. (Paris, Mallet-Bachelier, 1857 ).

[1o]. Construction de la tangente, du point de contact d'une droite avec son enveloppe pour certains lieux géométriques. Applications à la détermination du centre de courbure des coniques.

Nouvelles Annales de Mathematiques, Ière série, tome XVI (1857), pp. 322-332.

\section{858 .}

[II]. Sur la théorie des roulettes.

Bulletin de la Société Philomathique de Paris, Ve série, année 1858 , p. Io ${ }^{\mathbf{7} 7}$ ).

[12]. Constructions du centre de courbure de la courbe, lieu des points dont les distances à deux courbes données sont dans un rapport constant.

Annali di Matematica pura ed applicata, serie I, tomo I (18;8), pp. 364-369.

[13]. Construction des centres de courbure des lignes décrites dans le mouvement d'une figure plane qui glisse sur son plan.

Journal de l'École Impériale Polytechnique, Ière série, XXXVII Cahier (1858), pp. I79-19o.

\section{859 .}

[14]. Note de Géométrie infinitésimale.

Annali di Matematica pura ed applicata, serie I, tomo II (1859), pp. 208-21 3.

[I5]. Recherches géométriques relatives au lieu des positions successives des centres de courbure d'une courbe qui roule sur une droite.

Journal de Mathématiques pures et appliquées, $\mathrm{II}^{\mathrm{e}}$ série, tome IV (1859), pp. 93-104.

[16]. Note sur la théorie des polaires réciproques.

Nouvelles Annales de Mathématiques, Ière série, tome XVIII (1859), pp. 308-309.

[17]. Construction du centre de courbure de l'épicycloïde.

Ibid., id., id., pp. 37 I-376.

170) Per una correzione: Ibid., id., tome XVI (1857), p. 390.

I7x) Per quanto concerne le pubblicazioni della Société Philomathioue de Paris, la loro distribuzione in serie e il posto che vi occuparono da prima (1827-I83 I) il "Bulletin des Sciences》di Férussac e poi (1836-I875) il "Journal l'Institut", veggasi la prefazione del sig. Léon VaILlant alla Table générale par noms d'auteurs des Articles contenus dans les cinquieme, sixiène et septième séries des Bulletins (Paris, au Siège de la Société, r89o).

[G. B. G.]. 


\section{860 .}

[i8]. Application de la transformation par rayons vecteurs réciproques a l'étude de la surface enveloppe d'une sphère tangente $d$ trois sphères données.

Ibid., id., İ̉re série, tome XIX (I860), pp. 67-79.

[19]. Théorème de Géométrie.

Bulletin de la Société Philomathique de Paris, Ve série, année i 860 (séance du 5 mai 1860 ), p. 54 .

[20]. Sur la transformation par rayons vecteurs réciproques.

Ibid., id., id. (séance du Is décembre I860), pp. I04-106.

[2r]. Application de la transformation par rayons vecteurs réciproques à l'étude des anticaustiques.

Ibid., id., id. (séance du 22 décembre I860), pp. 107-108.

\section{I $86 \mathrm{I}$.}

[22]. Théorèmes de Géométrie.

Ibid., id., année I86r, p. 2 I.

[23]. Transformation par rayons vecteurs réciproques.

Nouvelles Annales de Mathématiques, İ̉re série, tome XX (I86I), pp. 218-2Ig.

[24]. Application de la transformation par rayons vecteurs réciproques à l'étude des anticaustiques.

Ibid., id., id., pp. 220-222.

[25]. Remarque \ l'occasion d'une communication récente de M. W. RoberTs. (Extrait d'une Lettre adressée à M. Serret).

Comptes rendus hebdomadaires des séances de l'Académie des Sciences (Paris), tome LIII $\left(2^{\mathrm{e}}\right.$ semestre $\left.186 \mathrm{I}\right)$, pp. $92 \mathrm{I}-922$.

\section{862 .}

[26]. Sur les arcs des courbes planes ou sphériques considérées comme enveloppes de cercles.

Journal de Mathématiques pures et appliquées, II série, tome VII (I862), pp. I2I-I35.

[27]. Note sur les rayons de courbure.

Nouvelles Annales de Mathématiques, II ${ }^{e}$ série, tome I (1862), pp. I23-125.

[28]. Sur les polygones plans inscrits et circonscrits aux courbes et remarques concernant le tracé des tangentes.

Applications d'Analyse et de Giométrie, par J.-V. Poncelet : Tome I (Paris, Mallet-Bachelier, I 862), Additions diverses, pp. 499-507.

[29]. Construction des tangentes à la courbe d'ombre de la surface de la vis à filets triangulaires.

Ibid., id., id., p. so8.

\section{863 .}

[30]. Recherches géométriques sur les longueurs comparées d'arcs de courbes différentes. Journal de l'École Impériale Polytechnique, İ̀re série, XL Cahier (I863), pp. $205-230$.

[3I]. Déterminer l'expression du rapport des rayons de courbure en deux points quelconques d'une courbe du $3^{\mathrm{e}}$ ordre.

Applicalions d'Analyse et de Géométrie, par J.-V. Poncelet : Tome II (Paris, Mallet-Bachelier, I864), Addition (mars I863) au II Cahier, Pp. I6I-I66. 


\section{864 .}

[32]. Sur les surfaces gauches.

Bulletin de la Société Philomathique de Paris, $\mathrm{VI}^{\mathrm{e}}$ série, année $\mathrm{I} 86_{4}$ (sćance du 2 avril I864), pp. 33-34.

[33]. Sur la surface gauche lieu des normales at une surface.

Ibid., id., id. (séance du 23 avril I864), pp. 42-43.

[34]. Sur la surface gauche lieu des normales principales d'une courbe gauche.

Ibid., id., id. (séance du 7 mai 1864), p. 58.

[35]. Sur la construction du centre de courbure des anallagmatiques.

Ibid., id., id. (séance du 6 août I864), p. I20.

\section{865 .}

[36]. Construction de la tangente en un point de la ligne d'ombre d'une surface de révolution.

Ibid., id., année r865 (séance du 4 février I865), pp. 9-10,

[37]. Sur ce problème: "Par un point donné mener des droites doublement tangentes à un tore ". (Par MM. Janin et Mannheim).

Ibid., id., id. (séance du 18 mars 1865 ), pp. 54-55.

[38]. Extrait d'une Lettre de M. Mannherm.

Nouvelles Annales de Mathématiques, $\mathrm{II}^{\mathrm{e}}$ série, tome IV (1865), pp. I33-135.

\section{866.}

[39]. Transformation par polaires réciproques des propriétés relatives aux rayons de courbure.

Journal de Mathématiques pures et appliquées, $\mathrm{II}^{\mathrm{e}}$ série, tome XI (I866), pp. I93-210.

[40]. Lettre concernant la collection de Mémoires de Lagrange, de Monge, de Laplace, etc., transmise par M. Biot à M. Edm. Bour, et léguée par celui-ci à l'Académie.

Comptes rendus hebdomadaire des séances de l'Académie des Sciences (Paris), tome LXII (rer semestre 1866), pp. 838-839.

[4I]. Sur le déplacement continu d'un corps solide; nouvelle méthode pour déterminer les normales aux lignes ou surfaces décrites pendant ce déplacement.

Ibid., id., pp. I386-I 390. - Journal de Mathématiques pures et appliquées, II ${ }^{\mathrm{e}}$ série, tome XI (1866), pp. 273-279.

[42]. Remarques sur un théorème de M. HaAG.

Bulletin de la Société Plilomathique de Paris, VI érie, année I866, pp. 27-28.

[43]. Sur une nouvelle méthode des normales aux lignes ou surfaces décrites pendant le déplacement continu d'un corps solide.

Ibid., id., id. (séance du 14 juillet I866), pp. 79-8I.

\section{7 .}

[44]. Détermination géométrique, pour un point de la surface des ondes, de la normale, des centres de courbure principaux et des directions des lignes de courbure. (Extrait d'une Lettre adressée à M. O. BonNET).

Comptes rendus hebdomadaires des séances de l'Académie des Sciences (Paris), tome LXIV ( ${ }^{\text {er }}$ semestre 1867 ), pp. I70-174. 
[45]. Construction géométrique, pour un point de la surface des ondes, des centres de courbure principaux et des directions des lignes de courbure. (Extrait d'une Lettre adressée à M. O. BonneT). Ibid., id., pp. 268-269.

\section{868 .}

[46]. Sur le déplacement d'une figure de forme invariable; nouvelle méthode des normales; applications diverses. (Extrait par l'Auteur).

Ibid., tome LXVI (I er semestre I868), p. 532 (séance du I6 mars I868). [Rapport de Chasles: Ibid., id., pp. 59I-598].-Bulletin de la Société Philomathique de Paris, VI ${ }^{e}$ série, année 1868 (séance du 28 mars 1868), pp. 52-6r.

\section{87 O.}

[47]. Sur un théorème qui présente de l'analogie avec celui de Meusnier.

Bulletin de la Société Philomathique de Paris, VI série, année 1870 , pp. I38-r 39.

[48]. Etude sur le déplacement d'une figure de forme invariable. Nouvelle méthode des normales; applications diverses.

Journal de l'École Impériale Polytechnique, Ière série, XLIII Cahier (I870), pp. 57-12I. Mémoires des Savants étrangers, tome XX (I 872 ), Pp. I-74.

[49]. Quelques résultats obtenus par la considération du déplacement infiniment petit d'une surface algébrique.

Comptes rendus hebdomadaires des séances de l'Académie des Sciences (Paris), tome LXX (rer semestre 1870), pp. I025-1028.

[50]. Recherches sur les pinceaux de droites et les normalies, contenant une nouvelle exposition de la théorie de la courbure des surfaces. (Extrait par l'Auteur).

Ibid., id., pp. 1074-1076.

[SI]. Détermination du plan osculateur et du rayon de courbure de la trajectoire d'un point quelconque d'une droite que l'on déplace en l'assujettissant à certaines conditions.

Ibid., id., pp. I215-1218.

[52]. Construction de l'axe de courbure de la surface développable enveloppe d'un plan dont le déplacement est assujetti à certaines conditions.

Ibid., id., pp. I259-I262.

[53]. Théorème de Géométrie.

Bulletin des Sciences Mathématiques et Astronomiques, Ière série, tome I (I870), p. I98.

\section{I $87 \mathrm{I}$.}

[54]. Démonstration géométrique d'un théorème de M. O. Bonnet.

Bulletin de la Société Philomathique de Paris, $\mathrm{VI}^{\mathrm{e}}$ série, année $187 \mathrm{I}$ (séance du 25 novembre 1871), pp. 228-229.

[55]. Démonstration géométrique d'une propriété de la transformation par rayons vecteurs réciproques.

Journal de Mathématiques pures et appliquées, II érie, tome XVI (1871), pp. 317-320.

[56]. Propriétés relatives aux déplacements infiniment petits d'un corps lorsque ces déplacements ne sont définis que par quatre conditions.

Comptes rendus hebdomadaires des séances de l'Académie des Sciences (Paris), tome LXXIII ( $2^{\mathfrak{e}}$ semestre 1871), pp. 1096-1098. 
[57]. Détermination simple et rapide d'une équation des surfaces du second ordre contenant six points donnés.

Bulletin des Sciences Mathématiques et Astronomiques, Ière série, tome II (1871), pp. 125-127.

\section{2.}

[58]. Généralisations du théorème de Meusnier.

Comptes rendus hebdomadaires des séances de l'Acádémie des Sciences (Paris), tome LXXIV ( ${ }^{\mathrm{er}}$ semestre I872), pp. 372-375.

[59]. Détermination de la liaison géométrique qui existe entre les éléments de la courbure des deux nappes de la surface des centres de courbure principaux d'une surface donnée.

Ibid., id., pp. 458-46I.

[6o]. Exposition sommaire d'une théorie géométrique de la courbure des surfaces.

Ibid., id., pp. 598-6or.

[6I]. Recherches géométriques sur le contact du troisième ordre de deux surfaces.

Ibid., id., pp. 856-860, 928-93I.

[62]. Théorèmes sur les courbes.

Bulletin de la Société Philomathique de Paris, VI érie, année 1872 (séance du 25 mai I872), p. 99.

[63]. Remarques sur une classe générale de surfaces, et en particulier sur la surface lieu des points dont la somme des distances à deux droites fixes est constante.

Bulletin des Sciences Mathématiques et Astronomiques, İ̉e série, tome III (I872), pp. I Ig-I 22.

[64]. Mémoire sur les pinceaux de droites et les normalies, contenant une nouvelle exposition de la théorie de la courbure des surfaces.

Journal de Mathématiques pures et appliquées, II ${ }^{\mathrm{e}}$ série, tome XVII (I872), pp. I09-I66.

[65]. Démonstration géométrique d'une proposition due à M. Bertrand.

Ibid., id., id., pp. 403-405.

[66]. Sur la surface gauche, lieu des normales principales de deux courbes.

Ibid., id., id., pp. 406-4I 7 .

[67]. Sur un modéle de vernier de vernier.

Comptes rendus hebdomadaires des séances de l'Académie des Sciences (Paris), t. LXXV $\left(2^{\mathrm{e}}\right.$ semestre I872), pp. I495-I497.

\section{873 .}

[68]. Sur les trajectoires des points d'une droite mobile dans l'espace.

Bulletin de la Société Mathématique de France, tome I (1873), pp. Io6-I14.

[69]. Sur les trajectoires des points d'une droite mobile dans l'espace.

Comptes rendus hebdomadaires des séances de l'Académie des Sciences (Paris), tome LXXVI (I ${ }^{\text {r }}$ semestre 1873 ), pp. $551-555$.

[70]. Propriétés relatives aux trajectoires des points d'une figure de forme invariable. Ibid., id., pp. 635-638.

[7I]. Deux théorèmes d'une nature paradoxale.

Association Française pour l'Avancement des Sciences, Compte rendu de la II $\mathrm{II}^{\mathrm{e}}$ Session (Congrès de Lyon, I873), pp. 82-84. 
[72]. Les normales aux surfaces trajectoires des points d'une figure de forme invariable rencontrent toutes deux mêmes droites. (Démonstration nouvelle de ce théorème). Ibid., id., pp. 95-97.

[73]. Quelques théorèmes montrant l'analogie qui existe entre les propriétés relatives aux surfaces décrites par les points d'une droite et les surfaces touchées par les plans d'un faisceau mobile.

Ibid., id., pp. 98-ror.

[74]. Note sur un modele de vernier.

Journal de Physique, tome II (1873), pp. 392-394.

\section{4.}

[75]. Démonstration géométrique de quelques théorèmes, au moyen de la considération d'une rotation infiniment petite.

Comptes rendus hebdomadaires des séances de l'Académie des Sciences (Paris), tome LXXVIII ( ${ }^{\text {er }}$ semestre 1874), pp. 633-637.

[76]. Deux théorèmes nouveaux sur la surface de l'onde. (Extrait d'une Lettre à M. O. BONNET).

Ibid., id., pp. $839-840$.

[77]. Construction directe du centre de courbure en un point de la section faite dans une surface par un plan quelconque.

Ibid., id., pp. 959-96I.

[78]. Construction directe du rayon de courbure de la courbe de contour apparent d'une surface qu'on projette orthogonalement sur un plan.

Ibid., id., Pp. I2I-12I7.

[79]. Détermination des relations analytiques qui existent entre les éléments de courbure des deux nappes de la développée d'une surface.

Ibid., tome LXXIX ( $2^{\mathrm{e}}$ semestre 1874), pp. 1328-1331.

[8o]. Sur la surface de l'onde.

Association Française pour l'Avancement des Sciences, Compte rendu de la $\mathrm{III}^{\mathrm{e}}$ Session (Congrès de Lille, 1874), pp. Ir68-1173.

[81]. Propriètés relatives à un faisceau de plans qui est mobile.

Ibid., id., pp. I I76-1 79 .

[82]. Construire la sphère osculatrice en un point de la courbe d'intersection de deux surfaces données.

Bulletin de la Société Mathématique de France, tome II (1874), p. I40.

[83]. Sur quelques problèmes relatifs à la théorie des surfaces.

Bulletin de la Société Philomathique de Paris, VI érie, année I874, p. 57.

\section{875 .}

[84]. Solutions géométriques de quelques problèmes, relatifs d la théorie des surfaces, et qui dependent des infiniment petits du troisième ordre.

Comptes rendus hebdomadaires des séances de l'Académie des Sciences (Paris), tome LXXX ( I er semestre 1875 ), pp. $54 \mathrm{I}-545$.

[85]. Solutions géométriques de nouveaux problèmes, relatifs à la théorie des surfaces, et qui deppendent des infiniment petits du troisième ordre.

Ibid., id., pp. 61g-622. 
[86]. Note à l'occasion de la Communication faite par M. Ribaucour dans la séance précédente.

Ibid., id., pp. 725-729.

[87]. Propriétés des diamètres de la surface de l'onde, et interprétation physique de ces propriétés.

Ibid., tome LXXXI ( $2^{\mathrm{e}}$ semestre I875), pp. 369-370.

[88]. Recherches sur la surface de l'onde.

Association Française pour l'Avancement des Sciences, Compte rendu de la IV ${ }^{\mathrm{e}}$ Session (Congrès de Nantes, 1875), pp. 167-173.

[89]. Propriétés des diamètres de la surface de l'onde et interprétation physique de ces propriétés.

Ibid., id., pp. 23 I-235.

[90]. On Three and Seven-bar Motion (two Letters communicated by Professor SyLVESTER).

Proceedings of the London Mathematical Society, Vol. VI (I874-75), pp. 35-36.

[9r]. Sur les surfaces trajectoires des points d'une figure de forme invariable dont le déplacement est assujetti à quatre conditions.

Journal de Mathématiques pures et appliquées, III érie, tome I (1875), pp. 57-74. - Mémoires des Savants étrangers, tome XXII, $\mathrm{N}^{\circ}$ I 2 (I876).

\section{876.}

[92]. Nouvelles propriètés géométriques de la surface de l'onde, qui s'interprètent en Optique.

Comptes rendus hebdomadaires des séances de l'Académie des Sciences (Paris), tome LXXXII (r er semestre I876), pp. 368-369.

[93]. Démonstration géométrique d'une relation due à M. Laguerre. Ibid., id., pp. 554-556.

[94]. Nouvelles propriétés optiques déduites de l'étude géométrique de la surface de l'onde.

Journal de Physique, tome V (1876), pp. I37-I44.

[95]. Remarque sur la surface de l'onde.

Association Française pour l'Avancement des Sciences. Compte rendu de la Ve Session (Congrès de Clermont-Ferrand, I 876), p. I 30 .

[96]. Construction pour un point de la courbe d'intersection de deux surfaces du centre de la sphère osculatrice de cette courbe.

Comptes rendus hebdomadaires des séances de l'Académie des Sciences (Paris), tome LXXXIII $\left(2^{\mathrm{e}}\right.$ semestre I876), pp. 1040-1042.

[97]. Nouvelles propriétés de quelques courbes.

Bulletin de la Société Mathématique de France, tome IV (1876), pp. I58-I59.

[98]. Note sur le tir lorsque le but est élevé au-dessus de l'horizon.

Revue d'Artillerie, tome VII (1876), pp. 338-342.

\section{877 .}

[99]. Sur le paraboloïde des huit droites.

Comptes rendus hebdomadaires des séances de l'Académie des Sciences (Paris), tome LXXXIV (I er semestre 1877 ), pp. 645-648. 
[Ioo]. Sur les surfaces dont les rayons de courbure principaux sont fonctions l'un de l'autre.

Ibid., id., pp. 932-935.

[IOI]. Sur le déplacement infiniment petit d'un dièdre de grandeur invariable. Ibid., id., pp. I373-1374.

[102]. Sur les surfaces dont les rayons de courbure principaux sont fonctions l'un de l'autre.

Bulletin de la Société Mathématique de France, tome V (1877), pp. 163-166.

[103]. Sur le paraboloïde des normales d'une surface réglée.

Ibid., id., pp. 190-193.

[104]. Sur les courbes ayant les mêmes normales principales et sur la surface formée par ces normales.

Comptes rendus hebdomadaires des séances de l'Académie des Sciences (Paris), tome LXXXV ( $2^{\mathrm{e}}$ semestre I877), pp. 212-216.

[105]. Nouveau mode de représentation plane de classes de surfaces réglées.

Ibid., id., pp. $788-79$ r.

[106]. Applications d'un mode de représentation plane de classes de surfaces réglées.

Ibid., id., pp. $847-850$.

[107]. Nouvelles applications d'un mode de représentation plane de classes de surfaces réglées.

Ibid., id., pp. 941-944.

[ro8]. Sur les plans tangents singuliers de la surface de l'onde et sur les sections faites dans cette surface par des plans parallèles à ces plans tangents.

Association Française pour l'Avancement des Sciences, Compte rendu de la $\mathrm{VI}^{\mathrm{e}}$ Session (Congrès du Havre, 1877), pp. I25-127.

[109]. Sur la surface de l'onde.

Ibid., id., pp. I67-168.

[iro]. Sur les normales de la surface de l'onde.

Ibid., id., pp. $175^{-1} 7^{-6}$.

\section{878 .}

[III]. On the Wave Surface.

The Messenger of Mathematics, Vol. VII (1878), pp. IOo-IOI.

[II2]. De l'emploi de la courbe représentative de la surface des normales principales d'une courbe gauche pour la démonstration de propriétés relatives à cette courbe.

Comptes rendus hebdomadaires des séances de l'Académie des Sciences (Paris), tome LXXXVI (i er semestre 1878 ), pp. I 254-i 256.

[I 3$]$. Sur les surfäces réglées.

Journal de Mathématiques pures et appliquées, III é série, tome IV (1878), pp. 57-60.

[I I4]. Nouvelle démonstration d'un théorème relatif au déplacement infiniment petit d'un dièdre, et nouvelle application de ce théorème.

Bulletin de la Société Mathématique de France, tome VI (1878), pp. 5-7.

[II ]. Démonstrations géométriques d'un théorème relatif aux surfaces réglées.

Ibid., id., pp. 7-9. 
[116]. Sur la surface de l'onde.

Association Française pour l'Avancement des Sciences, Compte rendu de la VII ${ }^{\mathrm{e}}$ Session (Congrès de Paris, I878), pp. 63-67.

[II7]. Transformation par polaires réciproques d'un pinceau de normales et extensions. Ibid., id., pp. I32-135.

[Ir8]. Construction de la normale à la surface trajectoire d'un point d'une figure de forme invariable dont le déplacement est assujetti à quatre conditions.

Ibid., id., pp. I52-154.

[II9]. Construction des centres de courbure principaux de la surface de vis à filet triangulaire.

Ibid., id., pp. 156-158.

[120]. Démonstrations directes de quelques propriétés connues relatives à la courbe enveloppe d'un segment de droite de longueur constante qui se meut dans un angle.

Nouvelles Annales de Mathématiques, II série, tome XVII (1878), pp. $32 \mathrm{I}-323$.

[I2I]. Construire les axes d'une ellipse, étant donnés deux diamètres conjugués. Ibid., id., id., pp. 529-535.

[r22]. Geometrical demonstration of a known Theorem relating to Surfaces.

The Messenger of Mathematics, Vol. VIII (1879), p. I22.

\section{879 .}

[I23]. Détermination géométrique des ombilics de la surface de l'onde.

Comptes rendus hebdomadaires des séances de l'Académie des Sciences (Paris), tome LXXXVIII (I ${ }^{\text {er }}$ semestre 1879), pp. 902-906.

[124]. Sur un mode de transformation des surfaces réglees.

Ibid., id., pp. I1 28-1 13r.

[125]. Transformation d'un pinceau de normales.

Ibid., id., pp. 1179-1183.

[i26]. Sur la surface de l'onde et sur la transformation d'un pinceau.

Ibid., id., pp. I248-1252.

\section{880 .}

[127]. Cours de Géonétrie descriptive de l'École Polytechnique, comprenant les éléments de la Géométrie cinématique.

Première édition. Un volume grand in $-8^{\circ}$, de $\mathrm{xx}-460$ pages, illustré de 249 figures dans le texte (Paris, Gauthier-Villars, I880).

[128]. La surface de l'onde considérée comme surface limite.

Comptes rendus hebdomadaires des séances de l'Académie des Sciences (Paris), tome XC ( $\mathrm{I}^{\text {er }}$ semestre $\mathrm{I} 880$ ), pp. 97 I-974.

[129]. Nouvelle génération de la surface de l'onde et constructions diverses.

Ibid., id., pp. I333-1336.

\section{$188 \mathrm{I}$.}

[130]. Construction de la normale d̀ la surface de Glaisher.

The Quarterly Journal of Pure and Applied Mathematics, Vol, XVII (I881), pp. I47-149. 
[13I]. Constructions planes des éléments de courbure de la surface de l'onde.

Collectanea Mathematica nunc primum edita cura et studio L. CREMONa et E. BeLTRAMI (Mediolani, Ulrici Hoepli, MDCCCLXXXI), pp. 9I-I04.

[132]. Sur la surface de l'onde, et théorèmes relatifs aux lignes de courbure des surfaces du second ordre.

Proceedings of the Royal Society of London, Vol. XXXII (I88I), pp. 447-450.

[I33]. Sur les surfaces parallèles.

Proceedings of the London Mathematical Society, Vol. XII (188I), pp. I77-186.

\section{882 .}

[134]. Sur les surfaces homofocales du second ordre.

Proceedings of the Royal Society of London, Vol. XXXIII (I882), pp. 322-33 I.

[135]. Sur les centres de courbure principaux des surfaces homofocales du second ordre. Ibid., id., pp. 42I-425.

[136]. Sur la détermination, en un point d'une surface du second ordre, des axes de l'indicatrice et des rayons de courbure principaux.

Journal de Mathématiques pures et appliquées, III ${ }^{e}$ série, tome VIII (1882), pp. I67-I72.

[137]. Premiers éléments de la Géométrie descriptive ${ }^{\mathbf{1 7 2}}$ ).

Nouvelles Annales de Mathématiques, III ${ }^{\mathrm{e}}$ série, tome I (1882), pp. 385-400, 433-450.

[138]. Premiers éléments de la Géométrie descriptive.

Une brochure in $-8^{\circ}$, de pages 40 (avec Avertissement). (Paris, Gauthier-Villars, I882).

[I39]. Construire les axes d'une ellipse dont on connait deux diamètres conjugués.

The Messenger of Mathematics, Vol. XI (1882), pp. I75-177.

[140]. Théorèmes de Géométrie.

Ibid., id., pp. rgo-rgr.

[14I]. Théorèmes de Géométrie.

\section{883.}

Ibid., Vol. XII (1883), pp. I75-176.

\section{884 .}

[142]. Sur le déplacement d'un dièdre de grandeur constante. Ibid., Vol. XIII (I884), pp. I68-169.

\section{885 .}

[143]. Mémoire d'optique géométrique contenant la théorie du point représentatif d'un élément de surface réglée et son emploi, tant pour la démonstration nouvelle de théorèmes relatifs à la courbure des surfaces que pour la détermination plane des éléments des surfaces caustiques.

Atti della R. Accademia dei Lincei (Roma), serie IV, Memorie della Classe di Scienze Fisiche, Matematiche e Naturali, vol. I (I 885 ), pp. 520.5 46 . - Journal de Mathématiques pures et appliquées, IV ${ }^{\mathrm{e}}$ série, tome II (1886), pp. 5-48.

[I44]. Représentation plane relative aux déplacements d'une figure de forme invariable assujettie à quatte conditions.

Comptes rendus hebdomadaires des séances de l'Académie des Sciences (Paris), tome C (I er semestre I885), pp. 268-27I.

172) Questo articolo fu tradotto in russo dal Prof. Liguine di Odessa.

Rend. Circ. Matem. Palermo, t. XXVI ( ${ }^{\circ}$ semestre I908). - Stampato il 3 marzo 1908. 
[145]. Sur la polhodie.

Ibid., id., pp. 938-940.

[146]. Sur l'herpolhodie.

Ibid., id., pp. 963-966.

[147]. Note de Géométrie.

Nouvelles Annales de Mathématiques, III érie, tome IV (I885), pp. Io5-109.

[148]. Sur une droite qui se déplace de façon que trois de ses points restent sur les faces d'un trièdre trirectangle.

Bulletin des Sciences Mathématiques, II ${ }^{e}$ série, tome IX (I885), pp. 137-I40.

[149]. Liaison géométrique entre les sphères osculatrices de deux courbes qui ont les mêmes normales principales.

Proceedings of the London Mathematical Society, Series I, Vol. XVI (1885), pp. 273-276.

[I5o]. Note on linkages.

The Messenger of Mathematics, Vol. XIV (I885), pp. 20-2r.

[I5I]. On the Wave Surface.

Ibid., id., pp. r8g-190.

\section{886.}

[I52]. Cours de Géométrie descriptive de l'École Polytechnique, comprenant les éléments de la Géométrie cinématique.

Deuxième édition. Un volume grand in $8^{\circ}$, de $\mathrm{xx}-480$ pages, illustré de 256 figures dans le texte. (Paris, Gauthier-Villars, I886).

[153]. Théorie géométrique de l'hyperboloïde articulé.

Comptes rendus hebdomadaires des séances de l'Académie des Sciences (Paris), tome CII (I I $^{\text {er }}$ semestre i 886 ), pp. $253-256$.

[154]. Sur le théorème d'Ivory et sur quelques théorèmes relatifs aux surfaces homofocales du second ordre.

Ibid., id., pp. 310-313.

[155]. Sur la polhodie et l'herpolhodie.

Ibid., id., pp. 353-356.

[156]. Sur l'hyperboloïde articulé et l'application de ses propriétés à la démonstration du théorème de M. De Sparre.

Ibid., id., pp. sor-s04.

[157]. Note on the Wave Surface.

The Messenger of Mathematics, Vol. XV (I886), pp. 40-4I.

[158]. Note de Géométrie cinématique.

Proceedings of the Cambridge Philosophical Society, Vol. VI (I886), pp. 32-37.

I 888 .

[159]. Sur certains conoïdes et en particulier sur le conoïde de PLÜcKER.

Comptes rendus hebdomadaires des séances de l'Académie des Sciences (Paris), tome CVI (I er semestre 1888), pp. 820-823.

[160]. Développements de Géométrie cinématique.

Mémoires publiés par la Societé Philomathique à l'occasion du centenaire de sa fondation I788-I 888 (Paris, Gauthier-Villars, I888), pp. SI-62. 
[I6I]. Applications de Géométrie cinématique.

Věstník královské české společnosti náuk. Tr̛̉ida mathematicko-přŕrodovědecká (二 Sitzungsberichte der Königl. böhmischen Gesellschaft der Wissenschaften. Mathematisch-naturwissenschaftliche Classe) (Prag), Ročník I888, pp. 87-92.

[162]. Construire le centre de courbure de la développée d'une conique.

Mathematisch-naturwissenschaftliche Mitteilungen, herausgegeben von Dr. O. Böklen (Tũbingen), Bd. II (I888), pp. 133-I35.

[i63]. Une Lettre à M. De Longchamps.

Journal de Mathématiques spéciales à l'usage des candidats aux Écoles Polytechnique, Normale et Centrale, III ${ }^{e}$ série, tome II (I888), pp. 283-284.

\section{889 .}

[164]. Étude d'un déplacement particulier d'une figure de forme invariable par des procédés élémentaires et purement géométriques.

Rendiconti del Circolo Matematico di Palermo, tomo III (I889), pp. I 3 I-I 44.

[165]. Construction du centre de courbure de la développée de la courbe de contour apparent d'une surface que l'on projette orthogonalement sur un plan.

Proceedings of the London Mathematical Society, Series I, Vol. XX (1889), pp. 24 I-244.

[166]. Note sur un système de deux courbes planes (par un Ancien Elève de Mathematiques spéciales).

Nouvelles Annales de Mathématiques, III ${ }^{\mathrm{e}}$ série, tome VIII (1889), pp. 325-329.

[167]. Construire les axes d'une ellipse dont on donne deux diamètres conjugués (par un Ancien Elève de Mathématiques spéciales).

Ibid., id., pp. 329-330.

\section{890.}

[168]. Sur un mode de transformation en Géométrie cinématique.

Comptes rendus hebdomadaires des séances de l'Académie des Sciences (Paris), tome CX (I ${ }^{\mathrm{er}}$ semestre I 890), pp. 220-223.

[169]. Sur un mode de transformation en Géométrie cinématique.

Ibid., id., pp. 270-272.

[170]. Transformations en Géométrie cinématique.

Ibid., id., pp. 39I-394.

[17r]. Sur le déplacement d'un double cône.

Ibid., tome CXI ( $2^{\mathrm{e}}$ semestre I890), pp. 634-636.

[I72]. Sur un nouveau mode de déplacement d'un double cône.

Ibid., id., pp. 8r7-8r 9 .

[173]. Mémoire de Géométrie cinématique: Sur le déplacement d'une figure de forme invariable dont tous les plans passent par des points fixes.

Journal de l'École Polytechnique, Ière série, $\mathrm{LX}^{\mathrm{e}}$ Cahier (1890), pp. 75-88.

[I74]. Rayon de courbure d'une conique.

Bulletin de la Société Mathématique de France, t. XVIII (I890), pp. I55-160.

[I75]. Théorème de Géométrie cinématique. (Extrait d'une Lettre).

Nouvelles Annales de Mathématiques, III ${ }^{e}$ série, tome IX (I8go), pp. 227-228. 
[176]. Détermination géométrique du centre de courbure de l'ellipse de Cassini.

Mathematisch-naturwissenschaftliche Mitteilungen, herausgegeben von Dr. O. Böklex (Tübingen), Bd. IIl (I890), pp. 14-16.

[177]. Note de Géométrie à propos d'un théorème de M. Stewart.

The Messenger of Mathematics, Vol. XIX (I890), pp. I78-180.

\section{89 I.}

[I78]. Remarques sur le déplacement d'une figure de forme invariable dont tous les plans passent par des points fixes.

Comptes rendus hebdomadaires des séances de l'Académie des Sciences (Paris), tome CXII (I er semestre I89I), pp. 283-284.

[179]. Transformation de démonstration.

Ibid., id., pp. 475-477.

[180]. Sur les normales aux coniques.

The Messenger of Mathematics, Vol. XX (1891), pp. I-2.

[18I]. Sur une parabole liée à une conique par certaines propriétés remarquables.

Ibid., id., pp. $45-48$.

\section{892.}

[182]. Extrait d'une Lettre.

Nouvelles Annales de Mathématiques, $\mathrm{III}^{\mathrm{e}}$ série, tome XI (1892), pp. 43 I-433.

[183]. Extrait d'une Lettre.

Ibid., id., id., Exercices, pp. 18*-19*.

\section{4 .}

[184]. Principes et développements de Géométrie cinématique; ouvrage contenant de nombreuses applications à la théorie des surfaces.

Un volume in- $4^{\circ}$, de x-590 pages, avec 86 figures. (Paris, Gauthier-Villars et fils, 1894).

[185]. Nouvel emploi du conoïde de Plücker.

Comptes rendus hebdomadaires des séances de l'Académie des Sciences (Paris), tome CXIX ( $2^{e}$ semestre 1894), pp. 394-397.

[186]. Nouvelle démonstration d'une propriété de l'indicatrice.

Bulletin de la Société Mathématique de France, tome XXII (I894), pp. 219-220.

[187]. Sur une transformation du conoïde de Plücker.

Association Française pour l'Avancement des Sciences, Compte rendu de la XXIII ${ }^{e}$ Session (Congrès de Caen, I894), p. 207.

\section{895 .}

[188]. Une propriété générale des axoïdes.

Comptes rendus hebdomadaires des séances de l'Académie des Sciences (Paris), tome CXX $\left(1^{\text {er }}\right.$ semestre 1895$)$, p. 67 I.

[189]. Sur les lignes de coubute des surfaces du second ordre.

Bulletin de la Société Mathématique de France, tome XXIII (1895), pp. I-4.

[190]. Extrait d'une Lettre.

Nouvelles Annales de Mathématiques, III ${ }^{\mathrm{e}}$ série, tome XIV (1895), pp. 349-350.

[I9I]. Propriété nouvelle de l'indicatrice.

The Messenger of Mathematics, Vol. XXIV (I895) pp. 59-60. 
[192]. On the circle of curvature at any point of an ellipse.

Ibid., id., p. Igo.

[193]. Sur la rectification approchée de la circonférence.

Journal de Mathématiques élémentaires, XIX année (I895), p. I03.

\section{6.}

[194]. Propriété nouvelle de la surface de l'onde.

Comptes rendus hebdomadaires des séances de l'Académie des Sciences (Paris), tome CXXII ( $\mathrm{I}^{\text {er }}$ semestre $\mathrm{I} 896$ ), pp. 708-71 I.

[195]. Sur les surfaces apsidales.

Ibid., id., pp. 1 396-1 398.

[196]. Sur le paraboloïde des huit droites et les nappes de développées de surfaces.

Ibid., tome CXXIII ( $2^{\mathrm{e}}$ semestre 1896 ), pp. 983-986.

[197]. Sur la détermination, en un point d'une surface du second ordre, des axes de l'indicatrice et des centres de courbure principaux.

Journal de Mathématiques pures et appliquées, Ve série, tome II (I896), pp. 5I-j5.

[I98]. Sur le rapport des deux courbures d'une courbe gauche.

Bulletin de la Société Mathématique de France, tome XXIII (I895), p. I88.

[I99]. Note d propos de la Question 486.

Journal de Mathématiques spéciales, $\mathrm{XX}^{\mathrm{e}}$ année (I896), pp. I4I-143.

\section{897 .}

[200]. Rernarqua à propos du Mémoire précédent ( Sur la théorie de l'octaèdre articulé ", par M. RaOul Bricard).

Journal de Mathématiques pures et appliquées, $V^{e}$ serie, tome III (I897), pp. I49-I50.

[20I]. Sur les formules de Frenet.

Bulletin de la Société Mathématique de France, tome XXV (I897), pp. 4-5.

[202]. Note à propos d'un théorème connu de Géométrie.

Ibid., id., pp. 78-82.

[203]. Sur la déviation dans l'ellipse.

Nouvelles Annales de Mathématiques, III érie, tome XVI (1897), pp. 249-252.

[204]. Sur le tracé de l'anse de panier.

Ibid., id., pp. 404-408.

[205]. Démonstration relative à l'inverseur de HaRT.

The Messenger of Mathematics, Vol. XXVI (1897), p. I II.

[206]. Extrait d'une Lettre (au sujet de l'article relatif à la courbe dite fibre moyenne).

Journal de Mathématiques spéciales, XXI ${ }^{\mathrm{e}}$ année (I897), p. I60.

[207]. Extrait d'une Lettre.

Journal de Mathématiques élémentaires, $\mathrm{XXI}^{e}$ année (1897), pp. 66-67.

\section{898.}

[208]. Remarques sur les constructions géométriques.

The Messenger of Mathematics, Vol. XXVII (1898), pp. 18-19. 


\section{900.}

[209]. Autre démonstration d'un théorème de Laguerre.

Bulletin de Mathématiques spéciales, tome VI (1900), pp. 98-99.

I 902.

[210]. Note de Géométrie.

Nouvelles Annales de Mathématiques, IV érie, tome II (I902), pp. 337-343.

[21 I]. Complément à la Note de la page 337.

Ibid., id., id., pp. 481-482.

[21 2]. Autre démonstration du théorème de Feuerbach. [Canon].

Ibid., id., id., pp. 500-50r.

\section{3.}

[213]. Note de Géométrie cinématique.

Association Française pour l'Avancement des Sciences. Compte rendu de la XXXII ${ }^{\mathrm{e}}$ Session (Congrès d'Angers, I903), pp. I28-1 30 .

[214]. Démonstration de la construction trouvée par Hamilton pour déterminer le point où le cercle des neuf points d'un triangle touche le cercle inscrit. [Canon].

Nouvelles Annales de Mathématiques, IV érie, tome III (I903), pp. I3-is.

[215]. Sur le théorème de Schclcher.

Ibid., id., id., pp. I05-107.

[216]. Démonstrations du théorème de Villarceau.

Ibid., id., id., pp. 250-253.

[217]. Expression de la variation de longueur d'une normale.

Ibid., id., id., pp. $48 \mathrm{r}-483$.

[218]. A propos d'une question proposée.

Ibid., id., id., pp. $483-485$.

\section{4 .}

[219]. Construire en grandeur et en direction les axes d'une ellipse dont on connait deux diamètres conjugués.

Ibid., id., tome IV (1904), pp. 5-7.

\section{5.}

[220]. Nouvelles démonstrations du théorème de Feuzrbach. Première démonstration par M. Canon.

Ibid., id., tome V (1905), pp. 257-259.

\section{906.}

[22I]. Démonstration de la construction trouvée par Hamilton pour déterminer le point où le cercle des neuf points d'un triangle touche le cercle inscrit.

Ibid., id., tome VI (1906), pp. 226-228.

[222]. Note à propos de la Question 1960.

Ibid., id., id., pp. 228-233. 
[223]. Le théorème de Feuerbach et l'inversion.

Bulletin des Sciences Mathématiques et Physiques élémentaires, XII ${ }^{e}$ année (I906), p. 89.

[224]. Expression du carré de la distance de l'orthocentre $H$ d'un triangle $A B C$ au centre $I$ du cercle inscrit à ce triangle.

Ibid., id., p. $90^{\text {173). }}$

Madesimo-Issime, luglio-settembre 1907.

GiNO LORIA.

I73) Questi due ultimi articoli ([223] e [224]) furono dal MANnherm scritti e mandati alla Redazione del "Bulletin des Sciences Mathématiques et Physiques élémentaires " negli ultimi giorni di novembre 1906, quando l'illustre geometra e compianto amico stava per esser colto dal male che lo trasse alla tomba. Essi comparvero nel $\mathrm{N}^{\circ} 6$ (I5 dicembre 1906) di detto "Bulletin» pochi giorni dopo la sua morte: II dicembre 1906 !

[G.B.G.]. 